\title{
WIDEBAND RF BEAMFORMING: ARCHITECTURES, TIME-DELAYS AND CMOS IMPLEMENTATIONS
}

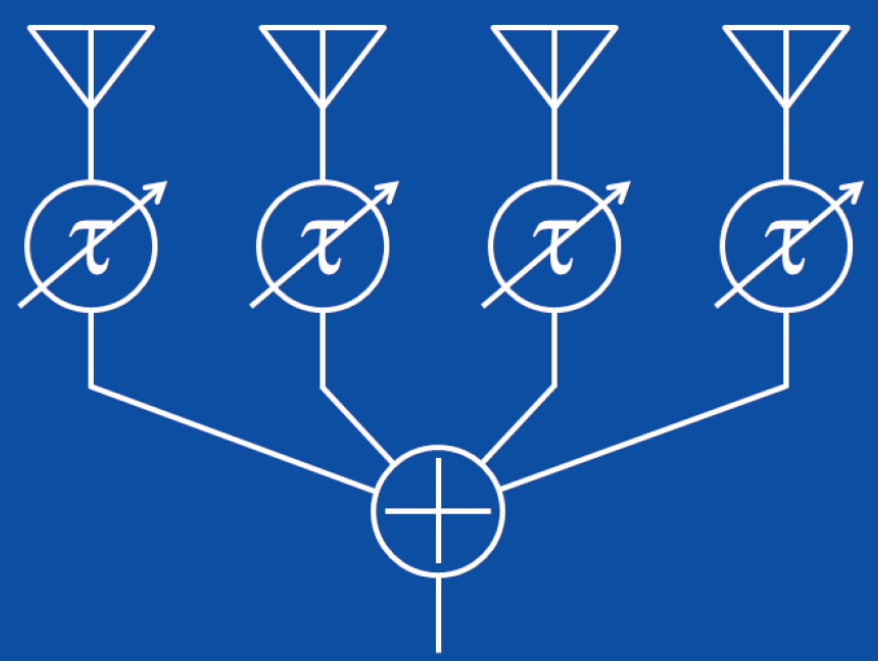

Seyed Kasra Garakoui 


\section{WIDEBAND RF BEAMFORMING: ARCHITECTURES, TIME-DELAYS AND CMOS IMPLEMENTATIONS}

Seyed Kasra Garakoui 


\section{Composition of the Graduation Committee:}

$\begin{array}{lll}\text { Prof.dr. } & \text { P.M.G. Apers } & \text { Universiteit Twente } \\ \text { Prof.dr.ir. } & \text { F.E. van Vliet } & \text { Universiteit Twente } \\ \text { Prof.dr.ir. } & \text { B. Nauta } & \text { Universiteit Twente } \\ \text { Dr.ing. } & \text { E.A.M. Klumperink } & \text { Universiteit Twente } \\ \text { Prof.dr.ir. } & \text { G.J.M. Smit } & \text { Universiteit Twente } \\ \text { Prof.ir. } & \text { A.J.M. van Tuijl } & \text { Universiteit Twente } \\ \text { Dr. } & \text { M. Matters-Kammerer } & \begin{array}{l}\text { Technische Universiteit } \\ \end{array} \\ \text { Prof.ir. } & \text { P. Hoogeboom } & \text { Eindhoven } \\ & & \text { Technische Universiteit Delft }\end{array}$

This research was financially supported by the Dutch Technology Foundation STW (07620)

CTIT Ph.D. Thesis Series No. 15-362

Centre for Telematics and Information Technology P.O. Box 217, 7500 AE, Enschede, The Netherlands

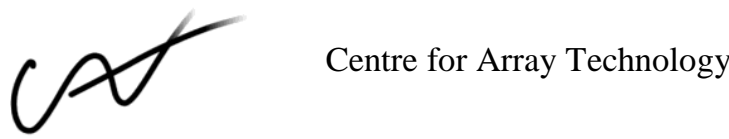

ISBN: 978-90-365-3901-2

ISSN: $\quad$ 1381-3617 (CTIT Ph.D. thesis Series No. 15-362)

DOI: $\quad 10.3990 / 1.9789036539012$

http://dx.doi.org/10.3990/1.9789036539012

Typeset with Microsoft Word. Printed by Gildeprint Drukkerijen, The Netherlands.

Copyright $@$ 2015, Seyed Kasra Garakoui, Enschede, The Netherlands.

All rights reserved. No part of this book may be reproduced or transmitted, in any from or by any means, electric or mechanical, including photocopying, microfilming, and recording, or by any information storage or retrieval system, without the prior written permission of the author. 


\title{
WIDEBAND RF BEAMFORMING: ARCHITECTURES, TIME-DELAYS AND CMOS IMPLEMENTATIONS
}

\section{DISSERTATION}

\author{
to obtain \\ the degree of doctor at the University of Twente, \\ on the authority of the rector magnificus, \\ prof.dr. H. Brinksma, \\ on account of the decision of the graduation committee, \\ to be publicly defended \\ on Friday the 26th of June 2015 at 16:45
}

by

Seyed Kasra Garakoui

born on the 21th of September 1974

in Rasht, Iran 
This dissertation has been approved by:

Promotor: prof.dr.ir. F.E. van Vliet

Co-promotor: prof.dr.ir. B. Nauta

Assistant-promotor: dr.ing. E.A.M. Klumperink 


\section{Contents}

Chapter 1 Introduction 1

1.1 Introduction 1

1.2 Phased array antenna systems __ 4

1.3 The implementation technology__ 6

1.4 Problem statement _ 7

1.5 Thesis outline _ 9

References _ 10

Chapter 2 Phased array antenna concepts $\quad 14$

2.1 Introduction 14

2.2 Phased array antenna systems___ 15

2.3 Analysis of the array factor _ 21

2.3.1 Main beam direction _ 23

2.3.2 Side lobes and grating lobe __ 25

2.3.3 Operating frequency _ 27

2.3.4 3dB Beam width__ 29

2.3.5 Number of nulls 29

2.3.6 Numbers of side lobes __ 29

2.3.7 Maximum required delay per channel ___ 30

2.4 Hierarchical Beamforming __ 30

2.5 Time delays _ 35

2.5.1 Transmission line _ 36

2.5.2 LC delay lines _ 37

2.5.3 $\mathrm{g}_{\mathrm{m}}-(\mathrm{R}) \mathrm{C}$ filters_ 38

2.5.4 Digital delays _ 38

2.5.5 Comparing delay blocks _ 39

2.5.6 Phase shifters__ 40

2.6 Beam squinting __ 43

2.6.1 Bandwidth limitation _ 46

2.6.2 Degrading the spatial interference rejection___ 47

2.6.3 Increase of side lobe levels __ 47

2.7 Wideband beamforming__ 49

2.8 Conclusion 52

References_ 53 
Chapter 3 A Quality Criterion for Delay Variations versus

Frequency

3.1 Introduction 56

3.2 A new criterion $\left(\mathrm{f}_{\varphi=0}\right)$ for time delay circuits_ 58

3.3 Time delay of blocks with frequency dependent group delay 62

$3.4 \mathrm{f}_{\varphi=0}$ for an RC and LC circuit__ 64

$3.5 \mathrm{f}_{\phi=0}$ of the cascaded delay _ 66

3.6 Simulation results 68

3.7 Conclusion 70

References__ 71

\section{Chapter 4 Phased-Array Antenna Beam Squinting Related to} Frequency Dependency of Delay Circuits 72

4.1 Introduction 72

4.2 Relation between $\Delta \theta$ and $\Delta \mathrm{f}$

$4.3 \mathrm{f}_{\varphi=0}$ : A criterion for delay versus frequency variations _ 77

4.4 Beam squinting formula _ 79

4.5 Beam squinting with all-pass delay cells _ 80

4.6 Example 82

4.7 Conclusion 83

References__ 84

Chapter 5 Frequency Limitations of First-Order $\mathrm{g}_{\mathrm{m}}-\mathrm{RC}$ All-pass

Delay Circuits $\quad 85$

5.1 Introduction 85

5.2 Analysis: The classical delay circuit _ 90

5.3 Analysis: The buckwalter delay circuit __ 93

5.4 Verification and design examples _ 96

5.5 Summary and conclusion _ 102

References__ 103

Chapter 6 Compact Cascadable $\mathrm{g}_{\mathrm{m}}$-C All-Pass True Time Delay Cell with Reduced Delay Variation over Frequency $\quad 105$

6.1 Introduction _ 105

$6.21^{\text {st }}$ order all-pass delay cell 109

6.3 The non-idealities of the delay cell ___ 111

6.4 Delay cell enhancements ___ 116

6.5 Beamforming System Design __ 125

6.64 channel wideband beamforming IC _ 126 
6.7 Chip Implementation and Measurements 129

6.8 Conclusions 138

References 139

Chapter 7 Summary and Conclusion 143

7.1 The work and the results 143

7.2 Original contribution 147

7.3 Recommendations for future work 147

List of Publications 



\section{Acknowledgments}

I would like to express my deep and sincere gratitude to my promotor professor Frank E. van Vliet, co-promotor professor Bram Nauta and my supervisor dr. Eric A.M Klumperink for guiding me during the project. Electronic circuit design and phased array antenna systems were only a part of the things that I have learned from them. I am proud to be their student and be gifted from their flames of wisdom. I would like to thank my colleagues at the university of Twente: Mr. Gerard Wienk, Mr. Henk de Vries and also especially thank to Dr. Michiel C.M. Soer, Mr. Paul Gaeradts and Mr. Mark Ruiter for their help and support during chip design, measurement and writing the thesis. Also thank to Dr. Daniel Schinkel for his always nice technical discussions. Also, I would like to thank Mrs. Gerdien Lammers for her help and encouragements. I like to thank my colleagues at Teledyne Dalsa Axiom IC company, especially the CEO, Mr. Gerard Hoogendijk for his help and support during the time that I am working there. My deep gratitude goes to my dear wife Celia Eslamieh Shomal. She has always stood by me during all the challenging times of the PhD period. Her love is a flame in my heart. At the end I would like to especially thank my mother Asieh Disahar. I will never forget her warm and smiley answer that she gave to me when I was 5 and asked her to solve my first mathematical assignment:" Think more! You can do it"

Seyed Kasra Garakoui,

Enschede,

The Netherlands

september, 2014 



\section{Samenvatting}

Een phased array antenne is een type antenne waarvan het stralingspatroon elektronisch te configureren is. Door het aantal stralingsbundels en hun richting te veranderen, is flexibele aanpassing mogelijk voor verschillende toepassingen en kan de kwaliteit van communicatiekanalen en van detectie geoptimaliseerd worden. Phased array antennes worden veel gebruikt voor radars om het aantal te detecteren doelen te vergroten en de scansnelheid enorm te verhogen, waarbij de mechanische beweging van antennes niet meer nodig is.

Circuits die vertragingen produceren vormen een essentieel onderdeel van een phased array antennesysteem. Hun vertragingsbereik, ruis, niet-lineariteit, bandbreedte, grootte, prijs en stroomverbruik zijn erg belangrijk voor phased array antennesystemen. Er bestaan verschillende methoden om in geïntegreerde circuits tijdvertragingen te realiseren, zoals met behulp van transmissielijnen, LC-vertragingslijnen, actieve filters, digitale vertragingscircuits (met digitale filters en logica) en fasedraaiers.

Dit proefschrift behandelt vertragingscircuits die geschikt zijn voor een compacte implementatie in standaard CMOS-processen, voor toepassingen van enkele honderden $\mathrm{MHz}$ tot enkele $\mathrm{GHz}$. Dit frequentiebereik is uitdagend omdat digitale signaalbewerking hiervoor in het algemeen te langzaam is en omdat het gebruik van spoelen en andere microgolf-componenten ongewenst is vanwege hun grote chipoppervlak.

Hiertoe is de kwantitatieve invloed van de frequentieafhankelijkheid van vertragingscircuits op de prestaties van een phased array antennesysteem bestudeerd. Het kental $f_{\varphi=0}$ is geïntroduceerd om vertragingsverandering ten gevolg van frequentieverandering te kwantificeren in vertragingscircuits. Er zijn 
vergelijkingen afgeleid die gebruik maken van de $f_{\varphi=0}$ van vertragingscircuits om verandering in de richting van stralingsbundels van een phased-array antenne te kwantificeren.

Een 1e orde all-pass filter kan binnen een beperkt frequentiegebied worden gebruikt als een benadering van een vertragingscircuit. Verschillende circuittopologieën van bestaande $\mathrm{g}_{\mathrm{m}}$-(R)C 1e orde all-pass filters worden behandeld en er wordt een vergelijkingsmethode geïntroduceerd om de filters onderling te vergelijken. Circuitsimulaties van $160 \mathrm{~nm}$ en $180 \mathrm{~nm}$ technologieën hebben laten zien dat deze circuits niet direct te cascaderen zijn en grote parasitaire capaciteiten hebben die hun bandbreedte beperken, waardoor de circuits ongeschikt zijn voor gebruik tot $3 \mathrm{GHz}$. Dit heeft geleid tot het idee voor een nieuwe topologie voor $1 \mathrm{e}$ orde all-pass filters.

Deze nieuwe topologie heeft aanzienlijk minder last van parasitaire effecten vergeleken met andere $1 \mathrm{e}$ orde all-pass topologieën waardoor de bandbreedte tot $5 x$ groter is vergeleken met eerdergenoemde 1e orde $\mathrm{g}_{\mathrm{m}}-(\mathrm{R}) \mathrm{C}$ filters. Verschillende technieken leidden tot een grotere bandbreedte, een lineaire fase, een nauwkeurigere afstelbaarheid met behulp van geschakelde capaciteiten en een te variëren versterkingsfactor.

Om de functionaliteit van het vertragingscircuit aan te tonen is het vertragingscircuit ontworpen en gebruikt in een implementatie van een 4-elements phased-array antennecircuit in $160 \mathrm{~nm}$ CMOS technologie. Metingen hiervan laten een bandbreedte zien van $1 \mathrm{GHz}$ tot $2.5 \mathrm{GHz}$. Hiermee is aangetoond dat $1 \mathrm{e}$ orde $\mathrm{g}_{\mathrm{m}}-\mathrm{C}$ filters geschikt zijn voor de realisatie van een breedbandig phased-array antennesysteem. 


\section{Abstract}

A phased array antenna is a kind of antenna which is electronically reconfigurable to realize different antenna beam patterns. Changing the number of antenna beams and their direction allows for flexible adaptation to different use scenarios and optimization of the quality of the communication links and remote sensing performance. Phased array antenna systems are extensively used in radars while the number of the detected targets and the speed of scanning the space dramatically increase via phased array antenna systems while also removing mechanically moving antennas.

Delay producing blocks are an essential part of phased array antenna systems. Their delay-range, noise, nonlinearity, bandwidth, size, cost and power consumption have a dominant effect on the phased array antenna systems. There are different physical methods for the realization of on-chip time delay blocks like transmission lines, LC delay lines, active filters, digital delay blocks (digital filters and gates) and phase shifters.

This thesis targets CMOS implementation of delay cells suitable for compact IC implementations in standard CMOS processes, in the hundreds of $\mathrm{MHz}$ to low $\mathrm{GHz}$ range. This frequency range is challenging as digital signal processing is usually not fast enough to cover this, while we like to avoid the use of inductors and other microwave components that tend to take large chip area.

For this purpose, the influence of the frequency dependency of the delay on the system performance of the phased array antenna system has been quantitatively studied. A criterion $\mathrm{f}_{\varphi=0}$ has been introduced to quantify the delay vs. frequency variations in delay blocks. 
Mathematical formulas have been found that use $f_{\varphi=0}$ of the delay blocks to quantify the beam direction variations in the phased array.

A 1st order all pass filter can approximately act as a delay block up to limited frequency bands. The circuit topologies of the existing $\mathrm{g}_{\mathrm{m}}-(\mathrm{R}) \mathrm{C}$ 1st order all-pass filters have been studied and a comparison method has been introduced to compare and benchmark them. The simulations on $160 \mathrm{~nm}$ and $180 \mathrm{~nm}$ technologies revealed that these circuits are not directly cascade-able and have large parasitic capacitors which limit their bandwidth and are therefore unsuitable for working up to $3 \mathrm{GHz}$. This has led us to the idea of a new topology for the 1st order all-pass filters.

The new topology has much less parasitic components compared to other 1st order all-pass topologies, therefore it has a bandwidth up to $5 \mathrm{x}$ more compared to the aforementioned 1st order $\mathrm{g}_{\mathrm{m}}-(\mathrm{R}) \mathrm{C}$ filters. Improvement techniques boosted the filter performance, for example, bandwidth extension, phase linearization, providing fine tune-ability via switched capacitors and also gain tuning.

In order to demonstrate the functionality of the delay block, it has been designed and used in implementation of a 4 antenna element phased array antenna chip at $160 \mathrm{~nm}$ technology and measurements results showed its bandwidth is wide enough to cover frequencies from $1 \mathrm{GHz}$ to $2.5 \mathrm{GHz}$. Measurement results proved that the 1 st order $\mathrm{g}_{\mathrm{m}}-\mathrm{C}$ filters are suitable for the realization of the wideband phased array antenna system. 


\section{Chapter 1 Introduction}

\subsection{Introduction}

Antennas are one of the essential parts of wireless communication systems. They act as transducers to convert guided waves to radiated waves or vice versa in transmitters and receivers [1], [2]. Their property directly affects the performance of wireless communication systems. For example, the spatial power density of the transmitted waves is directly defined by the beam pattern and the efficiency of the antennas [2]. Also, in receivers, the spatial selectivity for receiving waves, the spatial interference rejection and consequently the signal to interference ratio of the received signals strongly depend on the beam pattern and the efficiency of the antennas [1]. Besides, the bandwidth of the communication link directly depends on the bandwidth of the antennas both in transmitter and receiver side.

Many important specifications of an antenna are defined by the beam pattern of that antenna, for example, the direction, gain and the width of the main beam, the depths of nulls and the level of the side lobes [1]. The beam pattern of the antenna is largely defined by the physical shape of the antenna which conventionally is solid and unchangeable after being manufactured. In other words, the beam patterns of conventional antennas are fixed and unchangeable. On the other hand, in many wireless communication systems some degree of reconfigure-ability in the beam pattern of the antenna is essential. For this purpose a technique under the name of "beamforming" has been developed to provide re-configurability to the beam patterns of the antennas [1], [3]. By beamforming the spatial direction of the beams, the spatial direction of the nulls, the null depths and the side lobe levels can be electronically synthesized and reshaped by the operator of the antennas (these characteristics will be explained in the next 
chapter) [1], [4]. Beamforming can be implemented both in antennas at the receiver and the transmitter side. Beamforming has a wide range of applications, for example in spatial division multiple access techniques (SDMA) [5], [6], Smart antenna systems [7], [8], radars and remote sensing, satellite receivers and radio telescopes [9], [10].

Beamforming is exploited for spatial division multiple access systems (SDMA). The idea is to point the transmitted energy mainly in the desired direction to intensify the radiation density of electromagnetic waves from the transmitter toward the receiver and at the same time intensify the spatial selectivity of the receiver to reject spatial interference in the same frequency band. For example, SDMA is used in implementation of smart antenna systems (Fig.1.1). In smart antenna systems different transmitters and receivers operate simultaneously and beamforming facilitates the communication between each transmitter /receiver pair just by directing their antenna beams toward each other. This technique permits the simultaneous existence of several concurrent communication links beside each other, at the same frequency band without interfering each other. The SDMA technique dramatically increases the communication data rate without the need to increase the band-width of the system. 


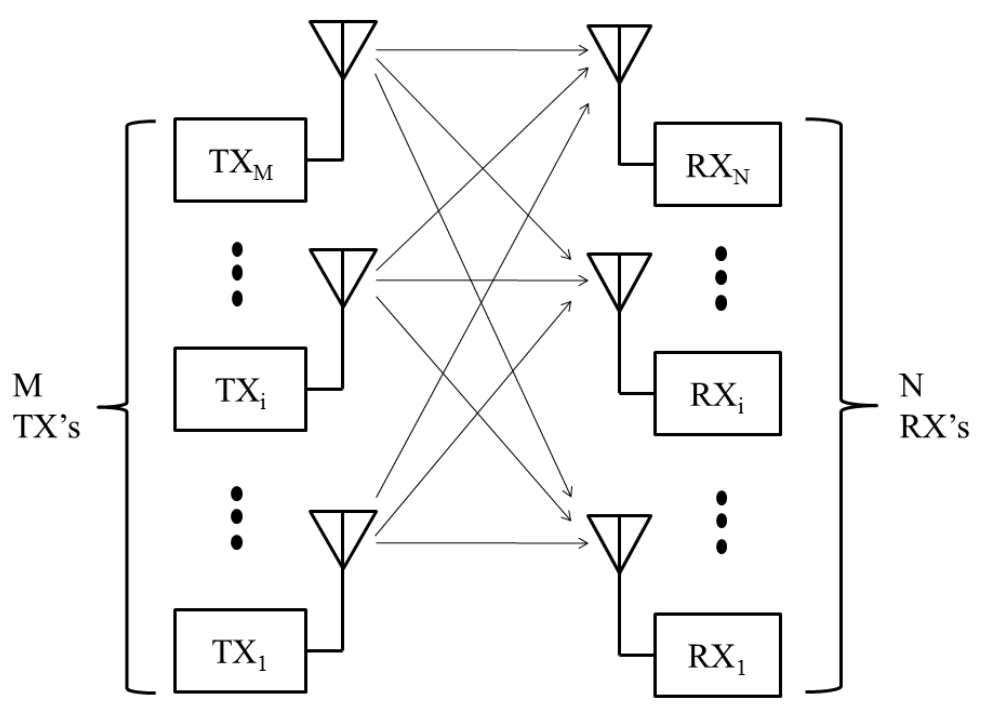

Fig. 1.1. An application example of SDMA using smart antenna systems with $\mathrm{M}$ transmitters and $\mathrm{N}$ receivers

Beamforming can dramatically relax the required dynamic range in receivers [5]. With this technique the received interferences from different spatial directions can be attenuated. It is done by setting nulls in the beam pattern of the antenna at the directions of the interferences (nulls are explained in next chapter). While the positions of interferences are dynamic, after sensing the directions of the interferences, beamforming facilitates the repositioning of the spatial direction of the nulls to guarantee that the spatial interferences are attenuated sufficiently. The attenuation of the interferences can relax the dynamic range requirements of the system. Otherwise strong interferences can desensitize the receivers. Especially, beamforming is useful in radio systems in which ADC's are used to digitize the received signals. The attenuation of large interferences relaxes the need to use high resolution (high dynamic range) ADC's. Relaxing the dynamic range requirement of the ADC directly reduces its costs and its power consumption [11], [12]. 
Another important application of beamforming is in radars. In radars the spatial direction of the main beam has to be continuously repositioned to scan the space [9]. Conventionally, this has been done via mechanical motors to rotate the total radar antenna structure, which is a slow, bulky, expensive solution. Via beamforming the beam can scan the space without the need to mechanically steer the antenna. Also, in contrast to the fixed beam antennas, via beamforming several beams can be made simultaneously to detect and lock on different targets. Beamforming in radars are resilient to jammers that dynamically change position. After detecting a strong jamming interference, beamforming can allocate nulls in the beam pattern in the direction of the interference with the purpose to attenuate the interference. If the jamming interferences change their location, beamforming radars again need to detect their direction and re-locate nulls toward them [1].

Beamforming allows for point to point communications without need for bulky mechanical motors. For example in satellite receivers conventionally mechanical motors have been used to steer the antennas toward spatial positions of different satellites which can be angularly quite near to each other (in the range of few degrees). Beamforming points the beam toward different satellites much faster without the need to rotate the antennas.

Space observation applications are another interesting application of beamforming systems. Nowadays, the radio telescope receivers that are made via beamforming systems can focus on extraterrestrial objects billions of light years away from us. Via beamforming very sharp beam widths are implementable to focus on faint objects [13].

\subsection{Phased array antenna systems}

Beamforming antennas are implementable via phased array antenna (PAA) systems [10]. A PAA system acts as a single antenna with an electronically reconfigurable beam pattern. The beam pattern of an antenna is the normalized plot of its far field radiated energy density as a function of the spatial direction [1], [10]. The PAA's are designed as receivers or transmitters of the electromagnetic waves. Their structure contains a set of individual antennas at different 
spatial positions and the signal of every individual antenna is processed via adjustable time delay blocks and gain stages [10]. Fig.1.2 shows the structure of a typical PAA receiver system. In PAA transmitter systems the combiner is replaced with a splitter and the placement direction of the amplifiers also changes. The total system acts as a spatial FIR filter on the radiated or received beam pattern. In other words, the beam pattern is the result of the summation of the time delayed, amplitude weighted replicas of the radiated or received waves. Via this method, different shapes of the beam patterns can be synthesized. The main beam of the beam pattern can be steered toward different spatial directions. The PAA system can provide more than one main beam simultaneously which is called multi-beam beamforming and has a wide range of applications in radars to detect multiple targets simultaneously [9]. It can allocate nulls in the beampattern at different spatial directions to attenuate the interferences. Besides, the side lobe levels (will be explained in the next chapter) of the beam pattern can be adjusted and limited to desired levels. Interestingly the re-configuration of the beam pattern can be done extremely fast because of the fast tune-ability of the time delays and the gain stages.

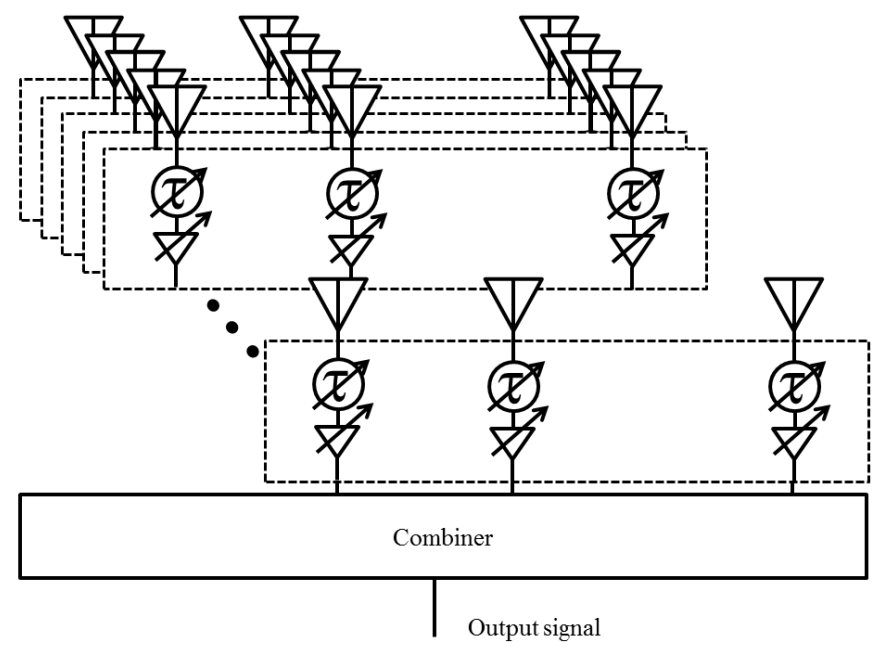

Fig.1.2. The structure of a PAA receiver system 
The beam pattern of a phased array antenna system is shaped via multiplication of two factors that are independent of each other: the element factor and the array factor [14]. The element factor is the beam pattern of the individual antenna elements of the phased array. The array factor is a function that depends on the spatial positions of the antenna elements and the amount of delay and amplification of each antenna element. Design of the element factor is a task for antenna designers and therefore is beyond the scope of this thesis. In the rest of the thesis the array factor is a main subject.

Time delay blocks are one of the essential parts of PAA systems because the spatial direction of the main beam is adjusted by the amount of delay in the time delay blocks [10], [14]. The required range of delay in time delay blocks is defined by the distance between the antenna elements, number of the antenna elements and the maximum angular steering of the beam. The tuning resolution of the delay blocks uniquely determines the angular steering resolution of the beam. Besides, the bandwidth, power consumption, noise, nonlinearity, delay variation per bandwidth, cost, size, reliability and reproducibility of the time delay blocks have dominant effects on the functionality, cost, size and reliability of the PAAs. Various methods exist to realize delay, for example, optical ring resonators [15], transmission lines [16], LC delay lines [17], active filters [18] and digital delay blocks [19]. Also "phase shifter blocks" [10] can approximate time delay blocks in narrow frequency bands.

\subsection{The implementation technology}

Beside bandwidth, cost and size, other main requirements in realization of phased array systems are reliability and reproducibility. PAAs require high degrees of matching between the sub-blocks of the phased array system. Poor matching between sub-blocks cause the requested beam directions, null depths and null directions in the beam patterns to be not achievable [10]. Otherwise, the delay and gain settings of individual antenna elements require complicated processes of calibration [20]. Therefore, the chosen implementation technologies must provide enough matching between sub-circuits of the phased array. 
CMOS IC technologies seem to be a good candidate. The digital and analog CMOS technology components and circuits perform the signal processing and data processing tasks in digital, mixed mode and communication systems [18], [21], [22]. The strong motivation from the digital electronic world for shrinking the size and increasing the unity current gain bandwidth $\left(\mathrm{f}_{\mathrm{t}}\right)$ results in very high speed CMOS transistors with a $\mathrm{f}_{\mathrm{t}}$ above $100 \mathrm{GHz}$. The high volume mass production allows for a low price. The model of the CMOS transistors provided by fabrication companies have been tested and measured in many ICs for several years which make the models quite reliable [22]. Therefore in $\mathrm{RF}$ communication systems it is attractive from a cost and size point of view to implement and integrate the RF circuits in CMOS technology on the same die beside the digital circuits [21]. Also, thanks to the high degree of matching between CMOS transistors, the sub-blocks of the phased array antenna systems can be implemented with a high degree of matching which finally simplifies the phased array calibration process. Of course not every part of the phased array antenna system can be on-chip implementable. For example antennas at low $\mathrm{GHz}$ frequencies have too large sizes to be implemented on-chip and only the sub-circuits are implemented on-chip.

\subsection{Problem statement}

The main goal of this thesis is to implement analog/RF beamforming systems in CMOS technology especially for wideband communication applications. The phased array antenna systems can be implemented via time delays or phased shifters. Phase shifters can imitate the operation of true time delays in limited frequency bands [10]. However, using phase shifters causes a phenomenon called beam squinting [10]. Beam squinting makes the beam direction frequency dependent and consequently causes bandwidth limitations in the beamforming system. Therefore for wideband beamforming systems, a straightforward method is to implement them via time delay circuits [10]. However, CMOS circuit designers have been encouraged to implement beamforming systems via phase shifters because high quality phase shifters are easily feasible in CMOS technology via switching mixers [23] , [24] which consequently cause beam squinting. 
Untill now, some ICs for wideband phased array antenna systems have been reported of which the delay circuits have been realized via LC (inductor-capacitor) delay lines or transmission lines [17] and [25]. Both of these techniques are area consuming. Especially for relative low frequency phased array systems (below $3 \mathrm{GHz}$ ) the required size of inductors or the lengths of the transmission lines make these techniques impractical for on-chip implementations.

Despite the mentioned benefits, CMOS technologies show some challenging characteristics in the realization of RF and phased array antenna systems. The intrinsic gain $\left(g_{m} r_{o}\right)$ of CMOS transistors degrades with shrinking [18]. Also the rail voltage (supply voltage) decreases by the downscaling of the CMOS feature size. Low supply voltages put a limitation on the number of the cascoded transistors fitting within the supply voltage. This has a direct influence on the quality of sub-circuits. For example, it limits the gains of the voltage and current amplifiers.

Using active filters [18], [21] is another method for the realization of on-chip time delay circuits, for example switched capacitor filters, opamp based filters and also $\mathrm{g}_{\mathrm{m}}-(\mathrm{R}) \mathrm{C}$ filters. The switching rate of the switched capacitor filters must at least be twice (Nyquist rate) the RF signal bandwidth they process for oversampling [19]. Also the opamps must have sufficiently high gain bandwidths to process the RF frequency bands. Therefore switched capacitor and opamp based techniques can be practical for the realization of time delay circuits operable at a few hundreds of megahertz but not for a few GHz. On the other hand, the $g_{m}-(R) C$ filters seem to be potentially good candidates for realization of time delay circuits at a few $\mathrm{GHz}$ frequencies. They benefit from the high unity gain frequencies $\left(\mathrm{f}_{\mathrm{t}}\right)$ of the modern CMOS technologies. $g_{m}-(R) C$ filter topologies can be quite wideband, certainly if there are no internal poles in them [26].

There are some reported first order all-pass $g_{m}-(R) C$ filter circuit topologies that approximately act as delay cells [27], [28] and [29]. Analysis and simulations of these circuit topologies at $160 \mathrm{~nm}$ and $180 \mathrm{~nm}$ CMOS technologies shows that these circuit topologies suffer from limited bandwidths up to around $1 \mathrm{GHz}$ [30]. The bandwidth limitation is because of the intrinsic parasitic capacitors in the circuit topologies. For higher bandwidths up to few $\mathrm{GHz}$ a new circuit 
topology is proposed. A suitable filter topology with small parasitic elements improves the bandwidths up to several GHz. Of course noise, nonlinearity and PSRR are very important considerations during the design of the filter topology.

This thesis describes the implementation of a wideband phased array antenna receiver system operating at low $\mathrm{GHz}$ frequencies (under $3 \mathrm{GHz}$ ) via $\mathrm{g}_{\mathrm{m}}-\mathrm{C}$ filters. For this purpose first a criterion $\left(\mathrm{f}_{\varphi=0}\right)$ is introduced to quantify the delay vs. frequency variations of the delay cells. Then, a mathematical expression is derived which uses $\mathrm{f}_{\varphi=0}$ of the individual delay cells to quantify the effect of the delay variations of the delay cells on the beam pattern of the phased arrays. Also a method is introduced to benchmark and compare the useful frequency range of the existing $\mathrm{g}_{\mathrm{m}}-\mathrm{C}$ and $\mathrm{g}_{\mathrm{m}}-\mathrm{RC}$ delay cells. Because the result of the comparisons between in literature reported $\mathrm{g}_{\mathrm{m}}-\mathrm{C}$ and $\mathrm{g}_{\mathrm{m}}-\mathrm{RC}$ delay cell topologies show that these topologies don't have enough bandwidth, therefore a new $\mathrm{g}_{\mathrm{m}} \mathrm{C}$ delay cell topology based on $1^{\text {st }}$ order all-pass filters is designed to provide enough bandwidth. The last part of this work describes the implementation of a wideband phased array antenna system via the designed $\mathrm{g}_{\mathrm{m}}-\mathrm{C}$ delay cell.

\subsection{Thesis outline}

Chapter 2 covers the operational mechanism and system level design of phased array antenna systems. Besides, the functionalities of the sub-blocks of the phased array are explained. Also in this chapter, the effects of non-idealities of sub-blocks on the total operation of the phased array are analyzed. Chapter 3 covers the criterion $\left(f_{\varphi=0}\right)$ to quantify the delay versus frequency variations of time delay cells. $f_{\varphi=0}$ quantifies how much a physical delay cell behaves similar to an ideal delay cell in a limited frequency band. Chapter 4 derives the mathematical expression which relates the bandwidth of the phased array antenna system to the $f_{\varphi=0}$ of its constituent delay cells. This expression will be used in later chapters to design a suitable delay cell to meet phased array specification requirements. In Chapter $5 \mathrm{a}$ method is proposed that uses $\mathrm{f}_{\varphi=0}$ to benchmark and compare different $\mathrm{g}_{\mathrm{m}}-\mathrm{RC}$ and $\mathrm{g}_{\mathrm{m}}-\mathrm{C}$ delay cells and find their useful bandwidth. Chapter 6 is about designing a $\mathrm{g}_{\mathrm{m}}-\mathrm{C}$ time delay cell that can work up to several $\mathrm{GHz}$ and about comparing that cell with other existing $\mathrm{g}_{\mathrm{m}}-\mathrm{RC}$ 
and $\mathrm{g}_{\mathrm{m}}-\mathrm{C}$ delay cells based on the method introduced in Chapter 5 . In addition, the design, implementation and measurement results of a phased array chip with the designed $g_{m}-C$ filter are reported. The final chapter, Chapter 7, contains a summary and conclusions.

\section{References}

[1] C. A. Balanis, in Antenna Theory: Analysis and Design, WileyInterscience; 3 edition , 2005.

[2] L. Besser and R. Gilmore, "Practical RF Circuit Design for Modern Wireless Systems, Volume I : Passive Circuits and Systems," Artech Print on Demand, 2003.

[3] M. A. Richards, in Fundamentals of Radar Signal Processing, Second Edition, McGraw-Hill , 2014, January.

[4] N. Fourikis, "Advanced Array Systems, Applications and RF Technologies," Academic Press, 2000.

[5] D. Tse and P. Viswanath, "Fundamentals of wireless communication," Cambridge University Press, 2005, July,.

[6] L.-K. Chiu, K.-Y. Lin and T.-H. Chang, "Robust Hybrid Beamforming with Phased Antenna Arrays for Downlink SDMA in Indoor $60 \mathrm{GHz}$ Channels," Wireless Communications, IEEE Transactions on, pp. 4542 - 4557, 5 August 2013.

[7] S. Bellofiore, C. A. Balanis, J. Foutz, A. S. Spanias, "Smart-Antenna Systems for Mobile Communication Networks. Part 1. Overview and Antenna Design," Antennas and Propagation Magazine, IEEE, pp. 145 - 154, June 2002.

[8] S. Bellofiore, C. A. Balanis, J. Foutz, A. S. Spanias, "Smart-Antenna Systems for Mobile Communication Networks. Part 2. Beamforming and Network Throughput," Antennas and 
Propagation Magazine, IEEE, pp. 106 - 114, August 2002.

[9] W. L. Melvin and J. A. Scheer, "Principles of Modern Radar," SciTech Publishing, 2012, September.

[10] R. J. Mailloux, "Phased Arrays in Radar and Communication Systems," in Phased Array Antenna Handbook, 2nd ed., Norwood, Artech house, 2005, ch.1, sec. 3, pp. 44-60.

[11] R. J. van de Plassche, "CMOS Integrated Analog-to-Digital and Digital-to-Analog Converters," Springer; 2nd edition, 2013, May.

[12] M. J. M. Pelgrom, in Analog-to-Digital Conversion, Springer; 2nd ed. , 2013.

[13] A. Sclocco, A. Varbanescu, J. Mol and R. van Nieuwpoort, "Radio Astronomy Beamforming on Many-Core Architectures," Parallel \& Distributed Processing Symposium (IPDPS), pp. 1105 - 1116, 2125 May 2012.

[14] H. J. Visser, Array and Phased Array Antenna Basics, Wiley, 2006.

[15] L. Zhuang, A. Meijerink, C. Roeloffzen, D. Marpaung and W. van Etten, "Novel ring resonator-based optical beamformer for broadband phased array receive antennas," IEEE Lasers and Electro-Optics Society, 2008. LEOS 2008. 21st Annual Meeting of the, pp. 20-21, 9-13 November 2008.

[16] G. Gonzalez, "Microwave Transistor Amplifiers: Analysis and Design," Prentice Hall; 2 edition, 1996, August..

[17] T. Chu, J. Roderick and H. Hashemi, "An Integrated UltraWideband Timed Array Receiver in $0.13 \mu \mathrm{m}$ CMOS Using a PathSharing True Time Delay Architecture," IEEE J.Solid-State Circuits, vol.42, no. 12, pp. 2834- 2850, Dec. 2007. 
[18] P. E. Allen and D. R. Holberg, "CMOS Analog Circuit Design," Oxford Series in Electrical and Computer Engineering, 2011, August.

[19] A. V. Oppenheim, R. W. Schafer and J. Buck, "Filter Design Techniques," in Discrete-Time Signal Processing, 2nd ed., New Jersey, Pretince-Hall, 1999.

[20] R. Sorace, "Phased array calibration," Antennas and Propagation, IEEE Transactions on (Volume:49, Issue: 4 ), pp. 517 - 525, 7 Augus 2012.

[21] B. Razavi, Design of Analog CMOS Integrated Circuits, New York: McGraw-Hill, 2001.

[22] Y. Tsividis and C. McAndrew, Operation and Modeling of the MOS Transistor, Oxford University Press; 3 edition, 2010, September.

[23] M. Soer, E. Klumperink, B. Nauta and F. van Vliet, "A 1.5-to$5.0 \mathrm{GHz}$ Input-Matched $+2 \mathrm{dBm}$ P1dB All-Passive SwitchedCapacitor Beamforming Receiver Front-End in 65nm CMOS," IEEE International Solid-State Circuits Conference Digest of Technical Papers, pp. 174-176, 19-23 Feb. 2012.

[24] S. Gueorguiev, S. Lindfors and T. Larsen, "A 5.2 GHz CMOS I/Q Modulator With Integrated Phase Shifter for Beamforming," Solid-State Circuits, IEEE Journal of (Volume:42, Issue: 9 ), pp. 1953 - 1962, September 2007.

[25] F. E. van Vliet, M. van Wanum, A. W. Roodnat and M. Alfredson, "Fully-integrated wideband TTD core chip with serial control," Gallium Arsenide applications symp., Munich, pp. 89-92, 2003.

[26] S. Pavan and Y. Tsividis, "High Frequency Continuous Time Filters in Digital CMOS," Springer, 2000, January. 
[27] P. Horowitz and w. Hill, "Unity-gainphase splitter," in The Art of Electronics, 2nd ed., New York, Cambridge University Press, 1999.

[28] K. Bult and H. Wallinga, "A CMOS analog continuous-time delay line with adaptive delay-time control," IEEE J. Solid-State Circuits, vol. 23, no. 3, pp. 759- 766, June 1988.

[29] J. Buckwalter and A. Hajimiri, "An active analog delay and the delay reference loop," IEEE RFIC Symp., 2004, pp. 17-20.

[30] S. K. Garakoui, E. A. M. Klumperink, B. Nauta and F. E. van Vliet, "Frequency Limitations of First-Order gm - RC All-Pass Delay Circuits," IEEE T. on Circuits and Systems II, vol. 60, no. 9, pp. 572576, Aug. 2013. 


\section{Chapter 2 \\ Phased array antenna concepts}

\subsection{Introduction}

A phased array antenna (PAA) system acts as a single antenna which can synthesize various beam patterns [1]. It is used both in transmitters and receivers. The basic structure of a PAA system consists of several antenna elements at different spatial positions. Each antenna element is connected to a time delay block or its equivalent to provide a controllable amount of delay to the antenna signals. Fig. 2.1 shows two basic PAA systems for the receivers and transmitters.

In this chapter first the basic structure, operational mechanism and terminology of the PAA are explained. Next, the hierarchical beamforming system is shown which gives the designers many degrees of freedom in designing the PAA systems. Following, the implementation limitations in the design of wideband PAA systems are studied, for example, the beam squinting phenomenon. Finally, we study different methods of physical implementation of the time delay cells with modern CMOS technologies ( $f_{t}$ up to $100 \mathrm{GHz}$ ) to decide which method is suitable for making delays up to $3 \mathrm{GHz}$ bandwidth. 


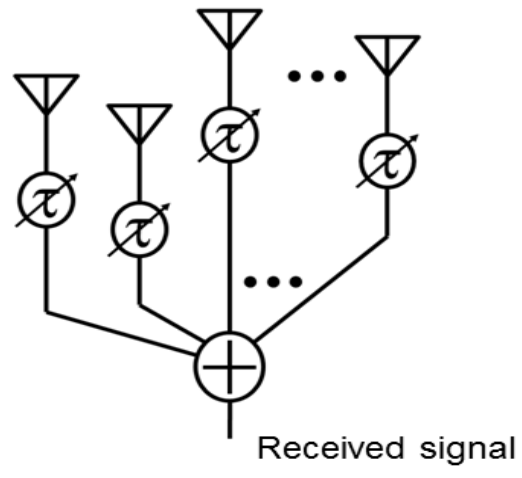

(a)

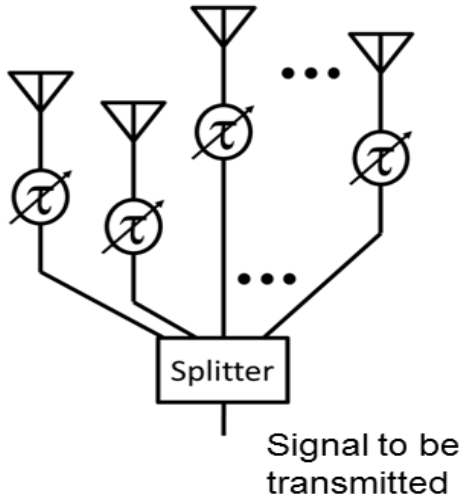

(b)

Fig. 2.1. Typical phased arrays (a) Receiver (b) Transmitter

\subsection{Phased array antenna systems}

Antennas with fixed physical shapes have fixed beam patterns. In contrast, it is possible to synthesize reconfigurable beam patterns via arrays of antennas (antenna elements). Antenna elements can be any antenna type, for example dipole, horn, helical or patch antennas. The antenna elements are positioned at different spatial points. Each antenna element is connected to a delay block. In phased array antenna receivers the signals of the antennas add together via a summation block and in phased array antenna transmitters the signals split to the antenna elements. Any circuit between each antenna element and the splitter or summation block will be called a part of the antenna channel. Because the main aim of this work is to make receivers, therefore the rest of the PAA systems in this thesis are PAA receivers and consequently contain only a summation block. The spatial arrangement of the antenna elements can have any form. It can be a line array, plane array or a three-dimensional array to provide different beam patterns. The basic and fundamental structure of which other arrangements of antenna elements can be constructed is a "linear equidistant phased array antenna" which is briefly called the "linear phased array" [1]. The linear phased array receiver is considered in the rest of the thesis. Fig. 2.2 shows a linear phased array receiver. It consists of ' $\mathrm{N}$ ' antenna elements in a line. The 
distance d between adjacent antenna elements are equal to each other. The electromagnetic waves are received by antenna elements from different spatial directions. The spatial directions of the waves are quantified with respect to the boresight which is the line perpendicular to the antenna elements line.

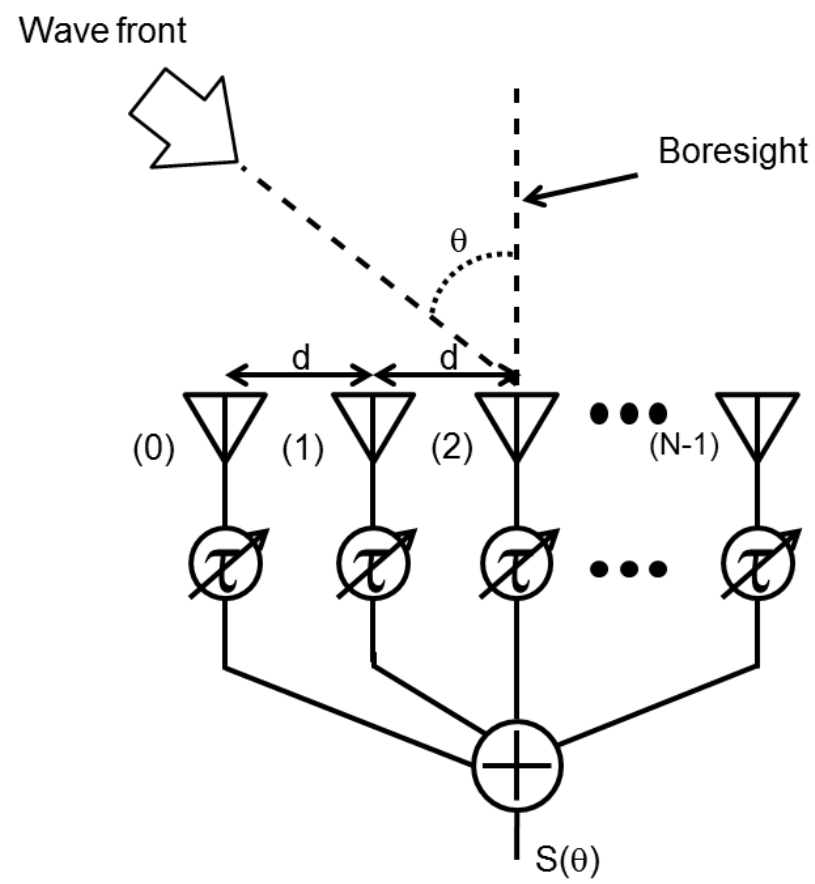

Fig. 2.2. A N-element linear equidistant phased array antenna receiver

Fig. 2.3 shows the operational mechanism of a linear phased array antenna receiver. To analyze the operational mechanism of the array independent to the beam pattern of the antenna elements, the antenna elements are considered isotropic. The result is a spatial radiation density pattern of the total array with isotropic antenna elements. 


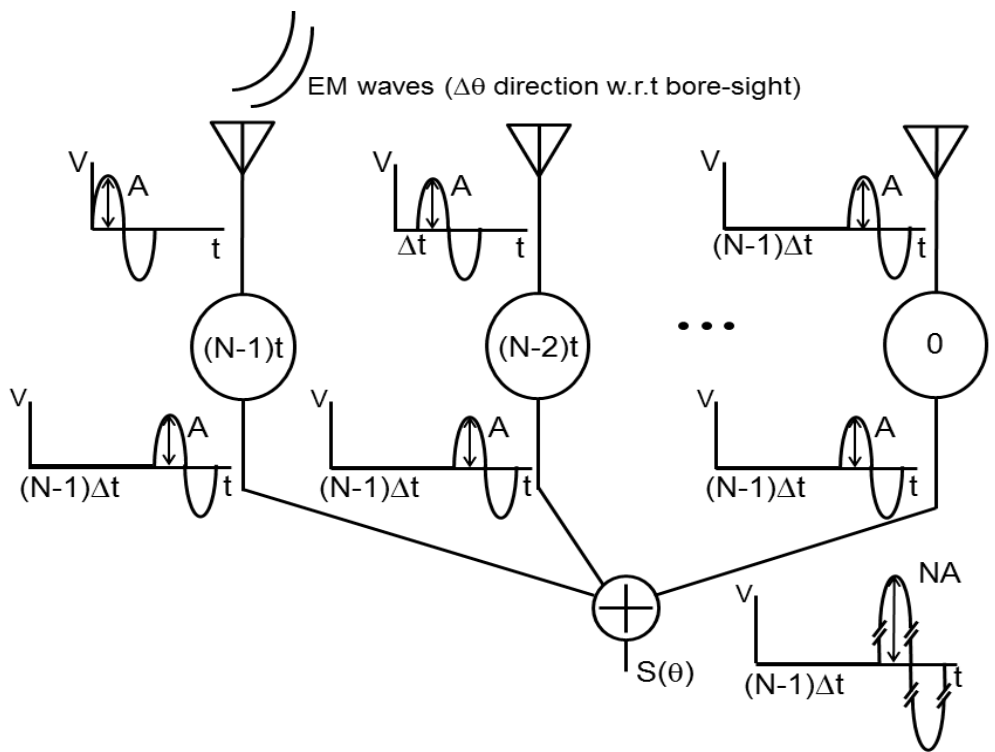

Fig. 2.3. Operational mechanism of a linear n-element equidistant phased array receiver

Waves come to the antenna elements from different spatial directions. Because of the relative spatial positions of the antenna elements their received signals have relative delays which depend on the direction of the waves and positions of the antenna elements. Time delay blocks compensate these relative delays and make the received signals of a particular direction (for example $\theta_{0}$ ) coherent. Therefore, after summing the signals together (in the summation block) the delayed version of the received signals from the direction $\theta_{0}$ add to each other coherently. In other words, after summation, the amplitude of the signals received from $\theta_{0}$ direction are ' $N$ ' (number of elements) times amplified in amplitude. The signals received from other directions after being delayed by time delay blocks are not aligned and partially or totally cancel each other after being added. In other words the received signals from the $\theta_{0}$ direction are amplified and at the same time the received signals from other directions attenuate. By retuning the values of the time delays to other values the constructive interference of the received signals occurs for other directions (for example $\theta_{1}$ ). 
The signal to noise power ratio of the received signal from the main beam direction is $\mathrm{N}$ times more than the signal to noise power ratio of each individual antenna channel from that direction. The improvement of the signal to the noise ratio is because the signals of all channels add coherently but the noises add together incoherently.

Fig. 2.4 shows two 3D plots that contain two perspectives of the spatial radiation density of a linear phased array in Cartesian coordinate system. Each consists of 4 isotropic antenna elements with equal amounts of delay at all antenna channels. The linear array is aligned along the z-axis with its center on the origin of the coordinate system. The operating frequency is $1 \mathrm{GHz}$ and the distances between antenna elements are chosen equal to half the wavelength $(\lambda \approx 30 \mathrm{~cm})$. The plot is normalized to 1 .

Fig. 2.5.a shows the top view of the spatial radiation density plot orthogonal to $x y$-plane $\left(\theta=90^{\circ}\right.$ plane in spherical coordinates $)$ and Fig. 2.5.b is the view orthogonal to $x z$-plane $(\varphi=0$ plane in spherical coordinates). 


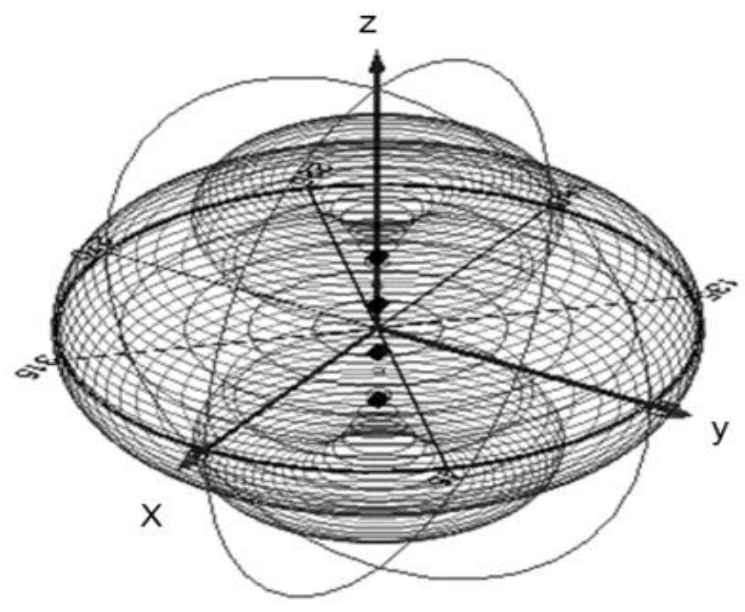

(a)

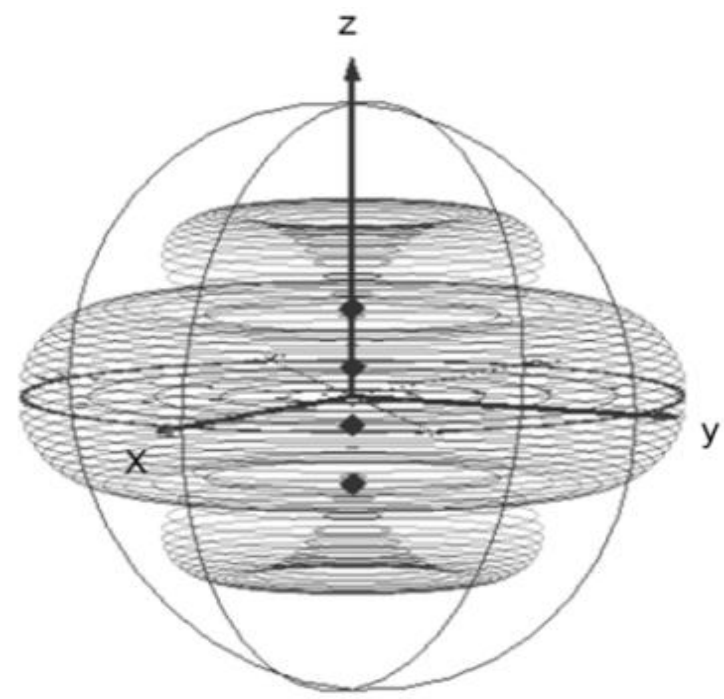

(b)

Fig. 2.4. The normalized 3D plot of the spatial radiation density of a linear phased array $(\mathrm{f}=1 \mathrm{GHz}, \mathrm{d}=0.5 \lambda)$ seen from two bird view perspectives 


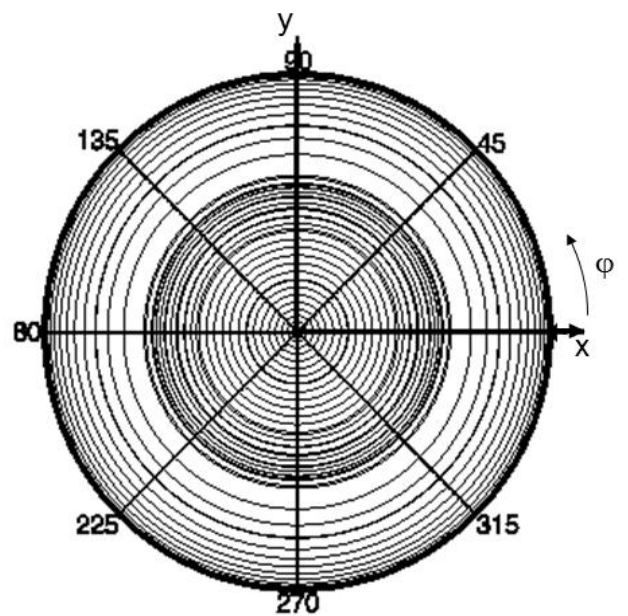

(a)

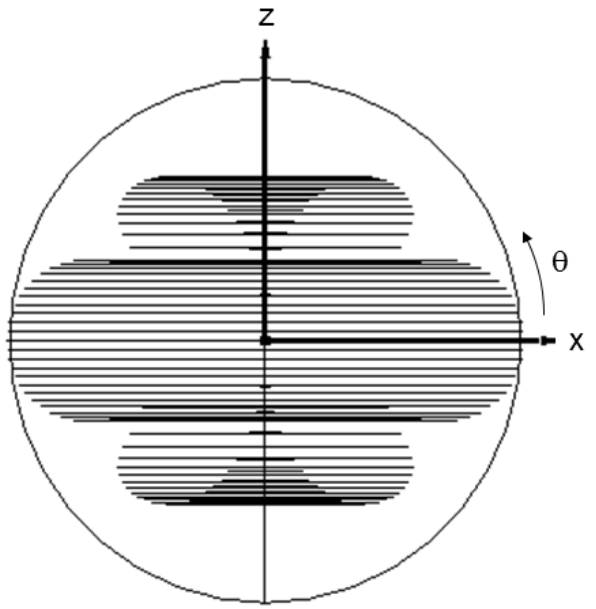

(b)

Fig. 2.5. The spatial radiation density of a linear phased array $(\mathrm{f}=1 \mathrm{GHz}, \mathrm{d}=0.5 \lambda)$ at two perspectives $(\mathrm{a})$ xy plane $(\mathrm{b}) \mathrm{xz}$ plane

As figure Fig. 2.5.a shows, in a spherical coordinate system the radiation power density plot is independent to $\varphi$. Therefore it is much easier to show that only in 2D Cartesian xz-plane or 2D spherical $(r, \theta)$ - 
plane. In the rest of this thesis only $2 \mathrm{D}(\mathrm{r}, \theta)$-planes are used to show the radiation power density plot. Because the power can vary several orders of magnitude by variation of $\theta$, the r-axis is shown in decibel. The radiation density plot of the linear phased array is symmetrical around the z-axis and therefore it is sufficient to show the pattern only for $\theta \in[-90,+90]$. Fig. 2.6 shows the radiation power density pattern of a 4 element linear phased array antenna $(d=0.5 \lambda)$ in $(r, \theta)$ plane. For the sake of visual simplicity $\theta$ has been mapped to a straight line.

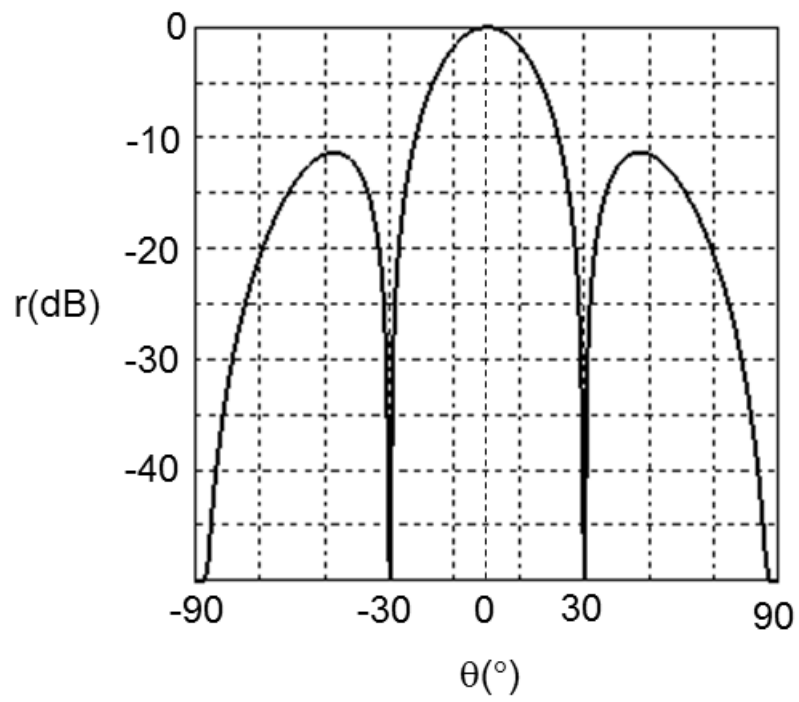

Fig. 2.6. The radiation power density pattern of a 4 element linear phased array antenna $(\mathrm{d}=0.5 \lambda)$

\subsection{Analysis of the array factor}

For a $\mathrm{N}$ elements linear phased array operating at frequency $\mathrm{f}_{0}$, to direct the beam toward $\theta_{0}$, the amounts of delay of the delay blocks in different channels $(\mathrm{k}=0, \ldots, \mathrm{N}-1)$ must be [1], [2], [3]: 
$\tau_{k}=(N-1-k) \frac{d}{c} \sin \theta_{0} \quad ; k=0, \ldots, N-1$

$\mathrm{k}$ : Antenna element index

$\mathrm{d}$ : inter elements distance

c: speed of the electromagnetic wave in free space

To plot the beam pattern of the phased array antenna system at operating frequency $f_{0}$, the array antenna system is exposed to sinusoidal waves of frequency $\mathrm{f}_{0}$ from all spatial directions. The received signal $(\mathrm{S}(\theta))$ is plotted vs. spatial arrival directions of the waves [1], [3]. The normalized power of the received signal is the beam pattern of the total antenna array [1]. $S(\theta)$ is expressed in eqn. 2.2. $S(\theta)$ consists of the multiplication of two parts: $S_{e}(\theta)$ and $S_{a}(\theta)$. The first part, $S_{e}(\theta)$, is called the element factor which indicates the contribution of the antenna elements in the total received signal. $S_{a}(\theta)$ is called the array factor which describes the spatial positions of the antenna elements and the amount of delay added to each antenna element (eqn. 2.3). The element factor and the array factor contribute to the beam pattern independent of each other. The array factor of the linear phased array can be simply depicted in $(r, \theta)$ (in spherical coordinates) plane because it is independent to $\varphi$.

$S(\theta)=S_{e}(\theta)\left|\sum_{k=0}^{N-1} \exp \left[j 2 \pi k \frac{d}{\lambda_{0}}\left(\sin \theta-\sin \theta_{0}\right)\right]\right|$

$S_{a}(\theta)=\left|\sum_{k=0}^{N-1} \exp \left[j 2 \pi k \frac{d}{\lambda_{0}}\left(\sin \theta-\sin \theta_{0}\right)\right]\right|$

A mathematical manipulation of the array factor and the use of element numbers $(\mathrm{N})$ simplifies eqn. 2.3 to eqn. 2.4 which is called normalized array factor $\left(\mathrm{S}_{\mathrm{a}, \mathrm{N}}(\theta)\right)$. It describes actually the beam pattern of an antenna array with isotropic elements. 
$S_{a, N}(\theta)=\frac{1}{N}\left|\frac{1-\exp \left[j 2 \pi N \frac{d}{\lambda_{0}}\left(\sin \theta-\sin \theta_{0}\right)\right]}{1-\exp \left[j 2 \pi \frac{d}{\lambda_{0}}\left(\sin \theta-\sin \theta_{0}\right)\right]}\right|$

Further mathematical analysis of the normalized array factor (eqn. 2.4) clarifies the most important characteristics and the specifications of the phased array, for example the beam direction, the 3-dB beam width, the number of nulls, the direction of the nulls, the information about the number of side lobes or existence of grating lobes and the required maximum and minimum amount of delays for the delay blocks. The array factor at each operating wave-length $\left(\lambda_{0}\right)$ is designed by choosing proper values of $\mathrm{N}$ and $\mathrm{d}$ and the reconfiguration of the array factor is done by changing the values of the delays or the amplifications at each antenna channel. Changing the amplitude at each antenna channel is done for tapering and that is not considered here [1].

\subsubsection{Main beam direction}

The main beam direction $\left(\theta_{0}\right)$ indicates the direction at which the normalized array factor has its maximum value which is equal to 1 ( 0 $\mathrm{dB}$ ) [1], [3]. As can be seen in eqn. 2.4, at $\theta=\theta_{0}$ both the numerator and denominator are zero and using L'Hopital's rule at $\theta=\theta_{0}$ [4] results in $\mathrm{S}_{\mathrm{a}, \mathrm{N}}\left(\theta_{0}\right)=1$. As an example, Fig. 2.7 shows the normalized array factor of a 4 element phased array antenna system with inter-element distances equal to $d=0.5 \lambda_{0}$. The main beam is directed toward $\theta_{0}=0$, $+30^{\circ}$ and $+60^{\circ}$ w.r.t. boresight. 
(a)

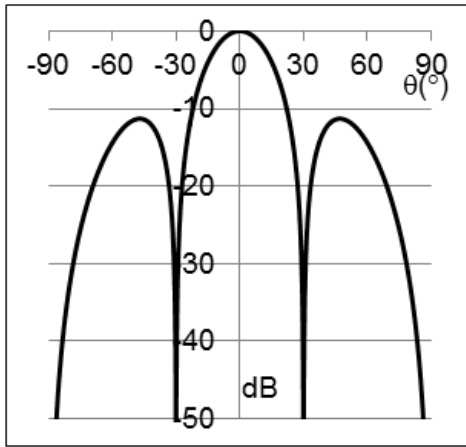

(b)

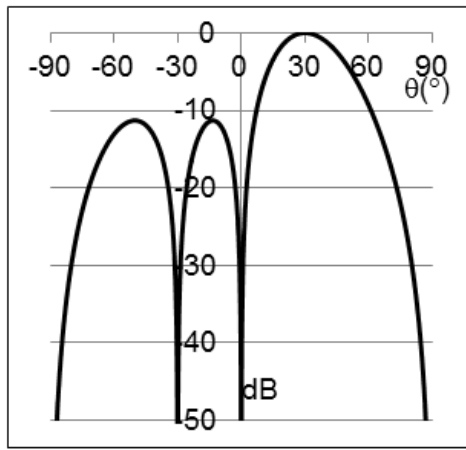

(c)

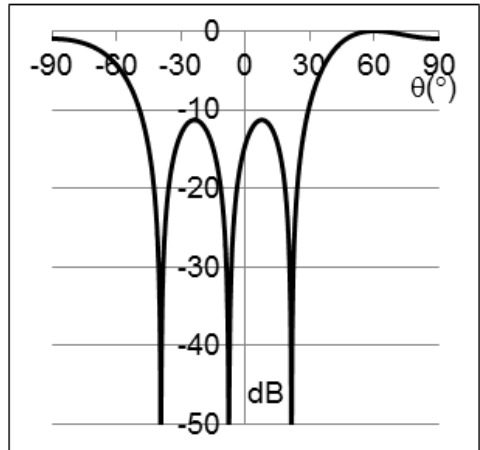

Fig. 2.7. Normalized array factor of a 4 antenna element linear phased array with $d=0.5 \lambda_{0}$, (a) $\theta_{0}=0$, (b) $\theta_{0}=+30$, (c) $\theta_{0}=+60$ 


\subsubsection{Side lobes and grating lobe}

Side lobes are local maxima of the array factor (excluding the main beam). The number of side lobes highly depends on the inter-element distances (d) and the operating wavelength. If the amplitude of a side lobe is equal to the amplitude of the main beam that side lobe is called a grating lobe. Grating lobes appear only when the denominator of eqn. 2.4 (normalized array factor) has more than one root in the spatial range $\left[-90^{\circ},+90^{\circ}\right]$. It is easily provable that for the condition of $d / \lambda_{0}>0.5$ grating lobes appear. Fig. 2.8 shows the array factor of a 4 antenna elements linear phased array with three values of $d / \lambda_{0}: 0.25$, 0.5 and 1 . For value of the $d / \lambda_{0}=1$ two grating lobes appear at $\theta= \pm 90^{\circ}$. 
(a)

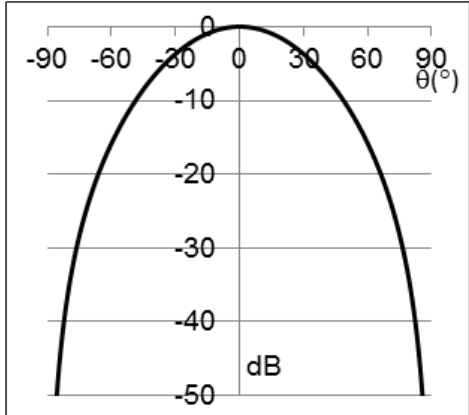

(b)

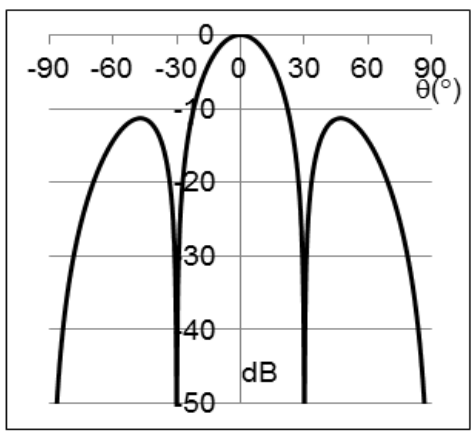

(c)

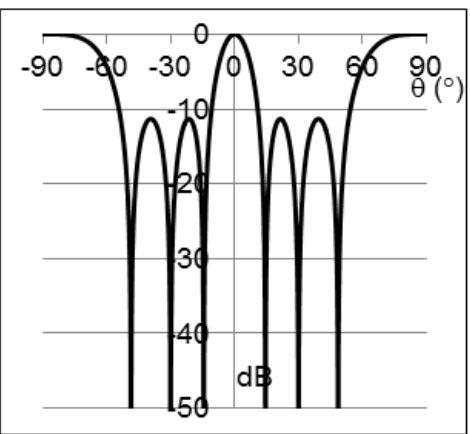

Fig. 2.8. Normalized array factor of a 4 antenna element linear phased array at $\theta_{0}=0$ for (a) $d=0.25 \lambda_{0}$, (b) $d=0.5 \lambda_{0}$, and (c) $d=\lambda_{0}$ 


\subsubsection{Operating frequency}

The operating frequency is the frequency of the waves exposed to the phased array system. By changing the operating frequency the wavelength $(\lambda)$ will change and so does the value of $d / \lambda$. This phenomenon does not affect the beam direction, however it has direct impact on the beam width, the angular position of nulls, the sidelobes and the existence and position of the grating lobes. Fig. 2.9 shows the effect of the frequency change on the array factor of a 4 antenna element array with relative inter-element distance of $\mathrm{d} / \lambda_{0}=0.5$ (the value of $\lambda_{0}$ is the wave length at frequency $\mathrm{f}_{0}$ ). The array factor is simulated for operating frequencies of $\mathrm{f}_{0} / 2$ (Fig. 2.9.b) and $2 \mathrm{f}_{0}$ (Fig. 2.9.c). For $f=f_{0} / 2$, compared to $f=f_{0}$, the value of $d / \lambda$ decreases and therefore the beam width will increase. For $f=2 f_{0}$, compared to $f=f_{0}$, the value of $d / \lambda$ increases which results in a narrow beam width at the cost of the emergence of grating lobes. 
(a)

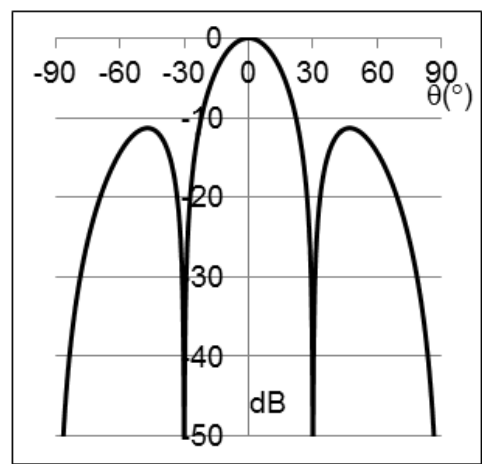

(b)

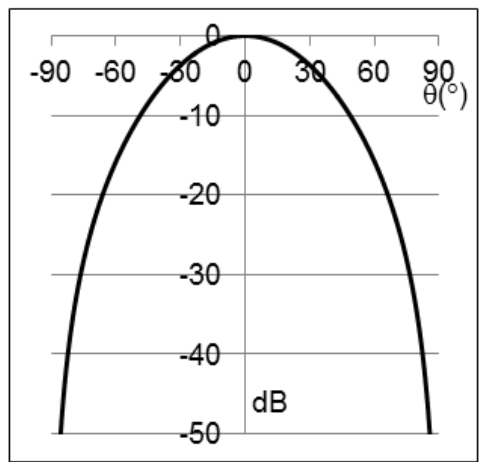

(c)

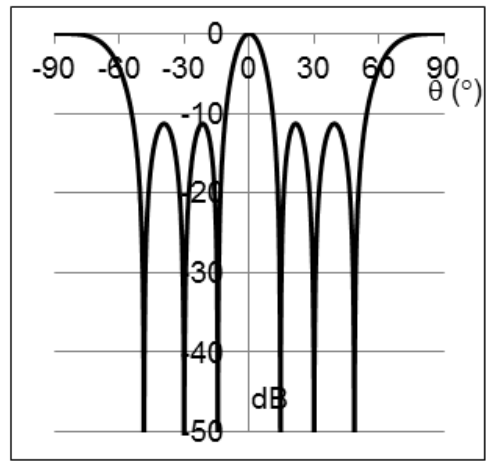

Fig. 2.9. Normalized array factor of a 4 antenna element linear phased array with $d / \lambda_{0}=0.5$ at (a) $f=f_{0},(b) f=f_{0} / 2$, (c) $f=2 f_{0}$ 


\subsubsection{3dB Beam width}

The $3 \mathrm{~dB}$ beam width is the angular range for which the normalized amplitude of the array factor is more than 0.707 (or -3dB) [1]. Eqn. 2.5 is an approximation of the $3 \mathrm{~dB}$ beam width of a $\mathrm{N}$ element linear array while the beam direction is toward $\theta_{0}$ [1]. Its reverse relation to $d / \lambda_{0}$ reveals that more relative inter-element distance results in a narrower beam width.

$3 d b$ Beam width $=\frac{0.88}{N \frac{d}{\lambda_{0}} \cos \theta_{0}}($ radian $)$

Although values of $d / \lambda_{0}$ larger than unity result in narrow beam widths, also grating lobes will appear. Therefore, the optimum value of the $d / \lambda_{0}$ is 0.5 which results in the narrowest possible beam pattern free of grating lobes [1]. In the rest of this thesis the value of $d / \lambda_{0}$ is considered equal to 0.5 .

\subsubsection{Number of nulls}

Nulls appear in the angular directions at which only the numerator of the equation 2.4 is zero (and the denominator is not zero). In equation 2.6 the integer values of $\mathrm{K}$ that satisfy the equation define the number of nulls in the steering range of $\theta \in\left[-90^{\circ}, 90^{\circ}\right]$ provided that the beam direction is $\theta_{0}$.

$$
-1-\sin \theta_{0}<\frac{K}{N} \frac{1}{\frac{d}{\lambda_{0}}}<1-\sin \theta_{0}
$$

\subsubsection{Numbers of side lobes}

The number of side lobes of the phased array is equal to [1]:

$$
\# \text { sidelobes }=(\# \text { nulls })-(\# \text { grating lobes })-1 \pm 1
$$

The term \pm 1 is decided based on the plot of the array factor. If in the plot just before end-fire angles $\left( \pm 90^{\circ}\right)$ there is a null, then -1 stays 
in the formula and if just before end-fire angles there is a side-lobe, then +1 stays in the formula.

\subsubsection{Maximum required delay per channel}

The maximum delay per channel is defined by three factors: the number of antenna elements $(\mathrm{N})$, the distance between antenna elements (d) and the maximum angle of beam direction $\left(\theta_{\max }\right)$. Their relation is shown in the following equation [3].

$$
\tau_{\max }=(N-1) \frac{d \cdot \sin \left(\theta_{\max }\right)}{C}
$$

(c is speed of EM waves in space)

\subsection{Hierarchical Beamforming}

As eqn. 2.8 showed, for arrays with a large number of elements the required maximum delays for the time delay blocks are large values. Because of the physical limitations (this will be explained later) making high frequency delay blocks with large amounts of delays is quite challenging. Therefore a method is used to relax the delay requirements for each antenna channel by the hierarchical combination of several phased array systems realized in different layers [2]. This method is called hierarchical beamforming. As an example, Fig. 2.10 shows a two layer hierarchical beamforming system. The first layer consists of several clusters of phased array antenna channels. The array factor of each beamforming cluster is an element factor for the next layer (second layer) of the phased array antenna system. 


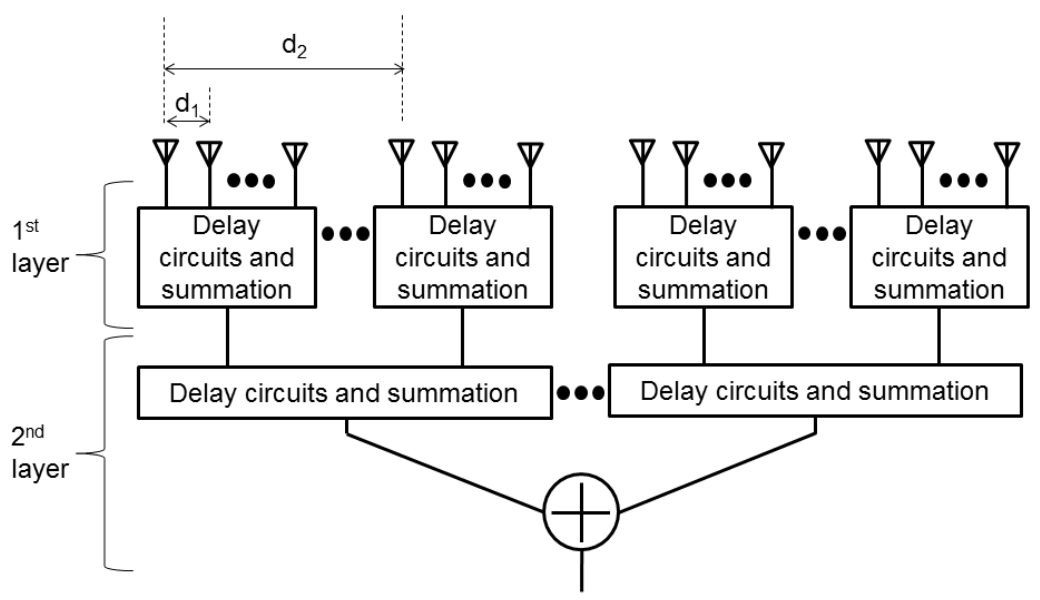

Fig. 2.10. Two layer hierarchical beamforming

As an example Fig. 2.11 shows a 16 antenna element two layer hierarchical beamforming phased array antenna system. The first layer consists of 4 sets of phased arrays, each has 4 antenna elements with relative inter-element distance of $d_{1} / \lambda_{0}=0.5$. The relative interelement distance for the second layer is $\mathrm{d}_{2} / \lambda_{0}=2$, because the distance between each two adjacent clusters of antennas $\left(d_{2}\right)$ is 4 times larger than $\mathrm{d}_{1}$. 


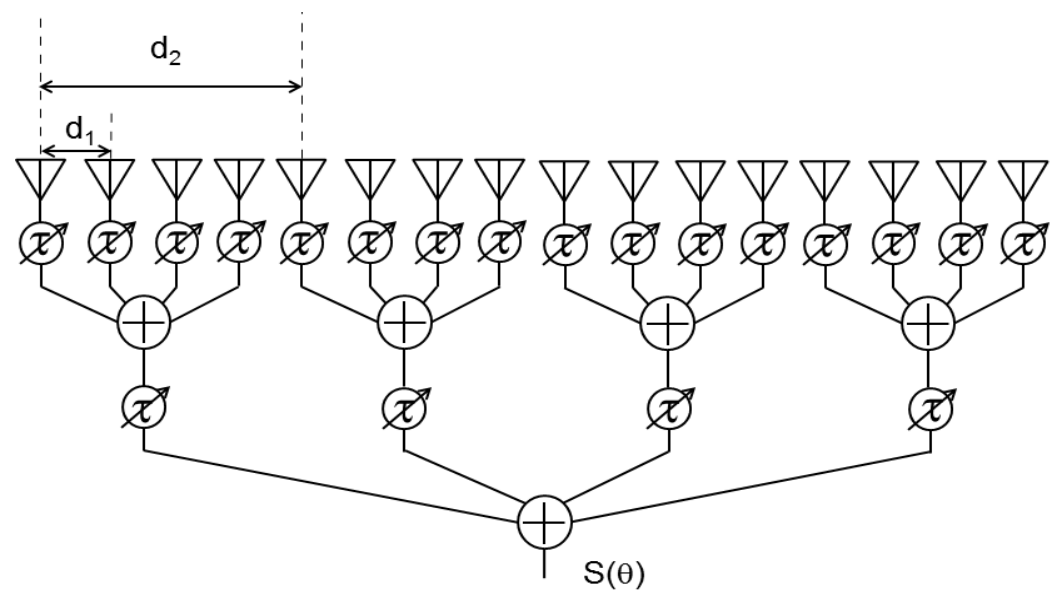

Fig. 2.11. A two-layer hierarchical beamforming

Fig. 2.12 shows the array factors of the first layer, second layer and the total phased array. The total array factor is the product of the array factors of the first layer and the second layer (layers are shown in Fig. 2.11). Because the length of the second layer is 4 times larger than the first layer, its beam width is much narrower but at the expense of the appearance of the grating lobes. As the total array factor shows, the grating lobes of the second layer are cancelled out by the nulls of the first layer while the beam width still remains narrow. Therefore, the normalized array factor of the total array is similar to the normalized array factor of a flat (not hierarchical) 16-element phased array. 
(a)

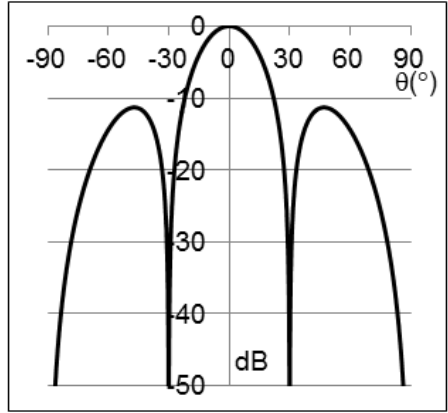

(b)

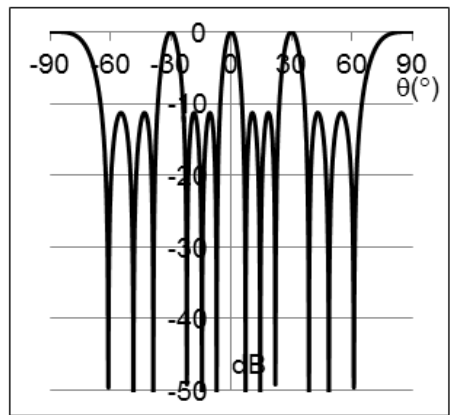

(c)

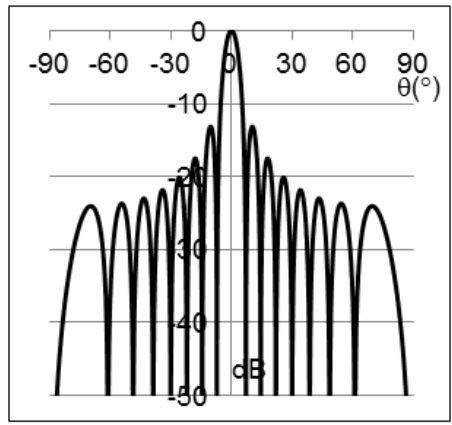

Fig. 2.12. The array factor of the (a) first layer, (b) second layer, (c)Total array 
In systems with hierarchical beamforming, the array factor of each layer acts as a spatial filter for the spatial interferences. This relaxes the high dynamic range requirements for the next layers. As an example Fig. 2.13 shows a 2-layer hierarchical beamforming system under exposure of two signals, a wanted signal that is weak and a strong interference. The first layer of beamforming allocates a null on the direction of the interference signal. Therefore the second layer receives the interference highly attenuated. This technique is quite helpful especially when the second layer is implemented via digital delay blocks (digital beamforming). It relaxes the wide dynamic range requirement for the ADCs [5], [6].

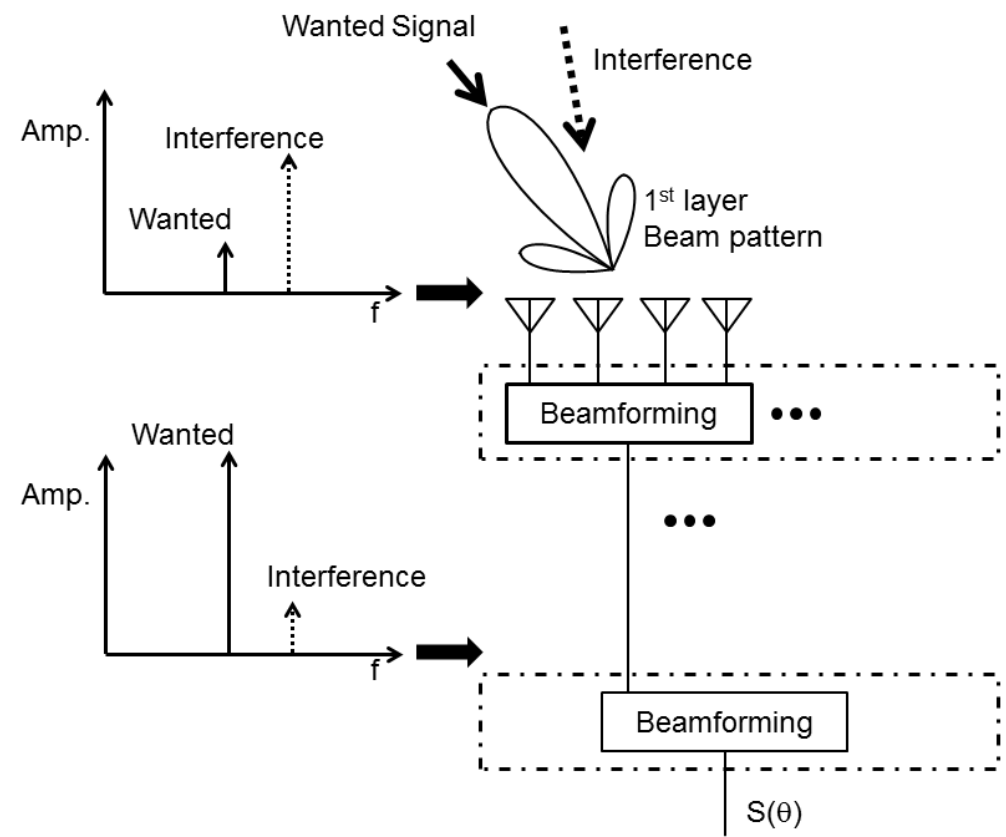

Fig. 2.13. The interference attenuation mechanism in hierarchical beamforming 


\subsection{Time delays}

A time delay block is a linear system or material which causes a time shift between its input and output signal. Ideally the amount of the delay is independent of the frequency of the signal, therefore, it gives an equal amount of delay to an input signal of any shape(Fig. 2.14)

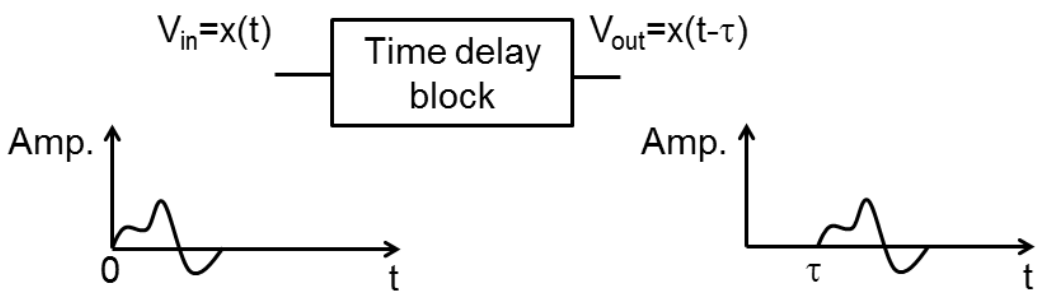

Fig. 2.14: Time delay block concept

The impulse response of the time delay block with delay amount of $\tau$ is: $h_{\tau}(t)=\delta(t-\tau)$. Therefore its transfer function in the Laplace domain is:

$H_{\tau}(j \omega)=\int_{0}^{\infty} \delta(t-\tau) e^{-j \omega t} d t=e^{-j \omega \tau}$

The gain and phase of the transfer function $\left(\left|H_{\tau}(j 2 \pi f)\right|\right.$ and $\angle H_{\tau}(j 2 \pi f)$ ) are shown in Fig. 2.15. This figure shows that the gain of the block is constant (unity) with respect to frequency and its phase is a linear function of frequency. The value of the delay is proportional to the slope of the phase transfer function (eqn. 2.10). 


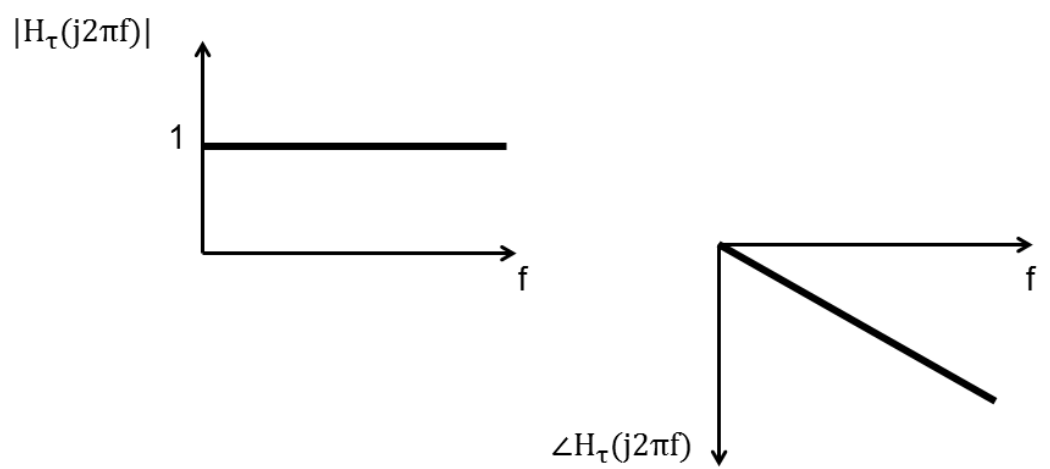

Fig. 2.15. The gain and phase transfer function of the time delay block

$\tau=-\frac{\angle H_{\tau}\left(j 2 \pi f_{0}\right)}{2 \pi f_{0}}$

In phased array systems the amount of delay in delay blocks must be tunable to provide beam direction adjustability. A tunable delay block still has a unity gain transfer function, but its phased transfer function shows a tunable slope.

Time delay blocks are implemented in either two ways: (a) Decreasing the propagation speed of the waves; (b) Increasing the physical length of the propagation path. Pursuing these physical properties, there are several methods reported for making delay blocks, for example, optical ring resonators [7], transmission lines [8], LC delay lines [9], active filters like $g_{m}-(R) C$ filters [10] and digital delay blocks [11]. Also "phase shifter blocks" [1] can approximate time delay blocks in narrow frequency bands. Some of these methods like optical ring resonators are beyond the scope of CMOS technology and therefore are not explained here.

\subsubsection{Transmission line}

A transmission line is a metal strip on the top of an isolated metal ground (Fig. 2.16). For a loss-less transmission line the speed of EM waves is equal to $v \approx \frac{1}{\sqrt{L C}}$, where $\mathrm{L}$ and $\mathrm{C}$ are elemental inductance and 
capacitance of the transmission line [12] (inductance and capacitance per unit length). In CMOS technology $v$ is approximately $2 / 3$ of the speed of light. The amount of delay of the transmission line directly depends on the length of the transmission line which is equal to $t_{x}=\mathrm{x} \sqrt{L C}$. The parameter $\mathrm{x}$ is the length of the transmission line. Therefore a weak point of transmission lines in CMOS technology is the need for a lot of area to make significant amounts of delay. A strong point is that the produced delay in this method is continuous and therefore implementing any value of delay is possible by tapping the signal at different lengths of the transmission line. The transmission lines theoretically don't burn power and also don't produce noise but in reality they cause signal loss and therefore a decreased signal to noise ratio.

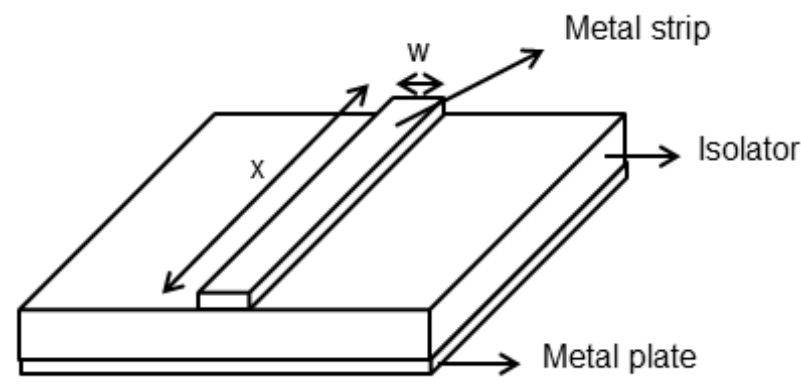

Fig. 2.16. Transmission line

\subsubsection{LC delay lines}

Fig. 2.17 shows an LC delay line. If the termination resistor is equal to $Z_{L}=\sqrt{L / C}$ then the amount of delay per $L C$ stage is equal to $\tau \approx \sqrt{L C}$ [12]. Large amounts of delay are achievable by large values of $L$ and $C$ which are quite area consuming [13]. Besides, the points where signals can be tapped are limited to the number of inductors; therefore, for having high delay resolutions many LC stages must be cascaded. The ideal LC delay line is passive, doesn't burn power and theoretically doesn't produce noise. But in reality the limited amount 
of Q (quality factors) for inductors implemented in CMOS technologies results in signal attenuation and therefore signal to noise degradation.

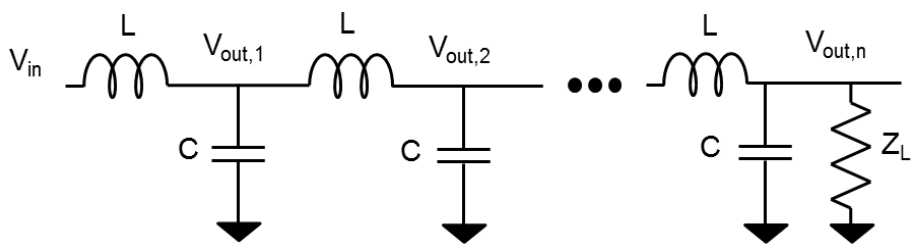

Fig. 2.17: LC delay line

\subsection{3 $\mathrm{g}_{\mathrm{m}}-(\mathrm{R}) \mathrm{C}$ filters}

$\mathrm{g}_{\mathrm{m}}-(\mathrm{R}) \mathrm{C}$ filters implemented in various topologies and with various degrees of complexity have been used to implement delay blocks [10], [14]. Because the structure of these filters is based on active elements, the trade-off between noise, frequency, delay and power consumption plays an important role in the design. The main advantage of these delay circuits is their high delay to area ratio. Compactness makes them suitable for low-RF frequency applications. Up to now no beamforming system implemented via time delays using $\mathrm{g}_{\mathrm{m}}-(\mathrm{R}) \mathrm{C}$ filters has been reported.

\subsubsection{Digital delays}

Digital delays are used in digital beamforming systems (Fig. 2.18) [15]. In digital beamforming the signals of each antenna channel are digitized via an Analog-to-Digital converter (ADC) and the beamforming function is realized via digitally implemented delays and a summation function. For example a well-known method for making digital delays is using IIR (infinite impulse response) filters [11]. The benefit of digital beamforming is the vast range of delays that this method can provide. By adjusting the coefficients of the digital filter small amounts of delay can be produced, which at the low side only are limited by jitter of the clock signals [6], [5]. On the other hand large amounts of delay are easily implementable with digital memories. In digital beamforming the main bottleneck is the ADC. The ADC must sample the input signal more than the Nyquist rate which is at least twice the maximum frequency of the input signal [11]. Also the 
precision of the ADC must satisfy the dynamic range requirements of the input signals. For processing RF signals the required sampling rate is dramatically high for the existing ADCs [5]. Precise ADCs with a high sample rate are highly power hungry and costly. Therefore the implementation of digital beamforming is limited to low and IF frequencies. In order to use digital delays for RF phased array antenna systems, first the RF signals must be down converted to IF. Besides, it may be necessary to relax the dynamic range requirements of the ADC via hierarchical beamforming at a layer before the digital beamforming. The mentioned solutions are promising but increase the complexity of the system and the required calibrations.

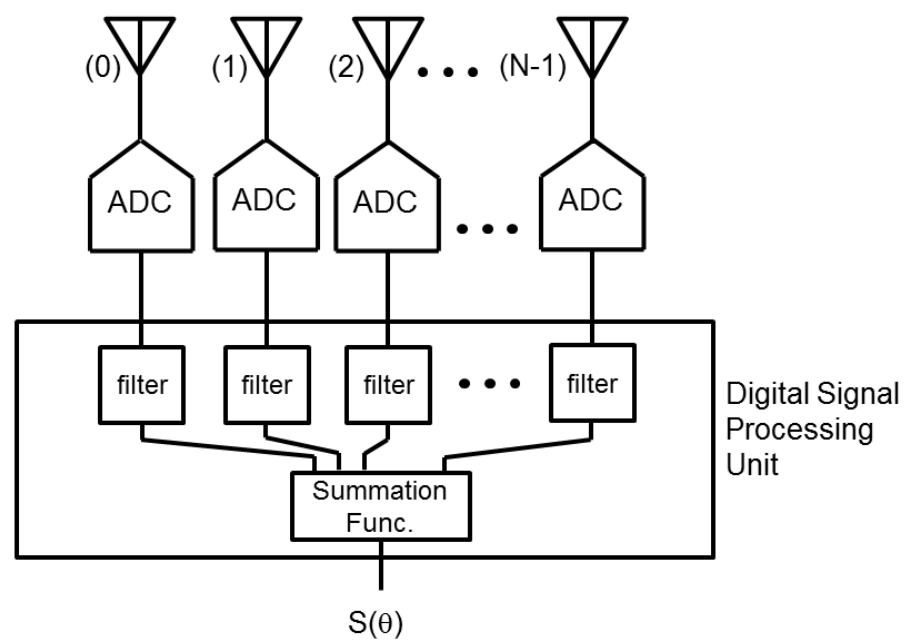

Fig. 2.18. Digital beamforming concept

\subsubsection{Comparing delay blocks}

Table 2.1 shows the comparison of the aforementioned delay implementation methods. We can see that the $\mathrm{g}_{\mathrm{m}}-(\mathrm{R}) \mathrm{C}$ delay circuits potentially offer a low cost, small size method for the implementation of an on-chip beamforming system, but their specifications highly depends on two factors: 1. Circuit topology 2. Implementation technology. Both factors will be analyzed in the following chapters. 


\begin{tabular}{|c|c|c|c|c|c|c|}
\hline & $\begin{array}{c}\text { Large } \\
\text { delay } \\
\text { S }\end{array}$ & Delay resolution & $\begin{array}{c}\text { For [1,3] } \\
\mathrm{GHz}\end{array}$ & $\begin{array}{c}\text { For } \\
>3 \mathrm{GH} \\
\mathrm{Z}\end{array}$ & $\begin{array}{c}\text { Are } \\
\mathrm{a}\end{array}$ & $\begin{array}{c}\text { Powe } \\
\text { rCons } \\
\mathrm{u} .\end{array}$ \\
\hline T-line & -- & ++ & -- & + & -- & ++ \\
\hline $\begin{array}{c}\text { LC } \\
\text { delay } \\
\text { line }\end{array}$ & -- & - & -- & + & -- & ++ \\
\hline $\begin{array}{c}\text { gm- } \\
\text { (R)C } \\
\text { filters }\end{array}$ & + & + & ++ & +- & ++ & +- \\
\hline $\begin{array}{c}\text { Digita } \\
\text { l } \\
\text { filters }\end{array}$ & ++ & ++ & -- & -- & ++ & -- \\
\hline
\end{tabular}

Table 2.1. A qualitative comparison of the different delay methods

\subsubsection{Phase shifters}

As aforementioned, besides the delay implementation methods, there is another alternative method for implementing beamforming, which is using phase shifters instead of time delays. Fig. 2.19 shows an ideal phase shifter block [1]. The phase shifter changes the phase of the input signal independent to frequency. Phase shifters can approximate the operation of the time delay blocks in limited bandwidths. Implementation of the phase shifters is quite compatible with CMOS technology because phase shifters can be simply realized via switching mixers and CMOS transistors are quite suitable for the switching function. However, next to shifting phase, mixers perform frequency shifting (up-conversion or down-conversion) on the input signal spectrum. The frequency shifting can also be used in RF to IF conversions in beamforming systems. Fig. 2.20 shows a phase shifter realized via a mixer. If the minus shift of the phase shift $(-\varphi)$ is applied to the local oscillator (LO) frequency, then the phase shift of the downconverted signal is equal to $\varphi$. 


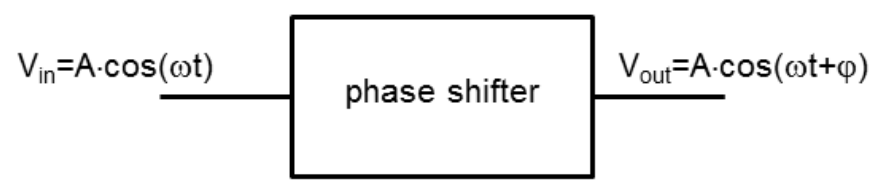

Fig. 2.19. An ideal phase shifter (block level view)

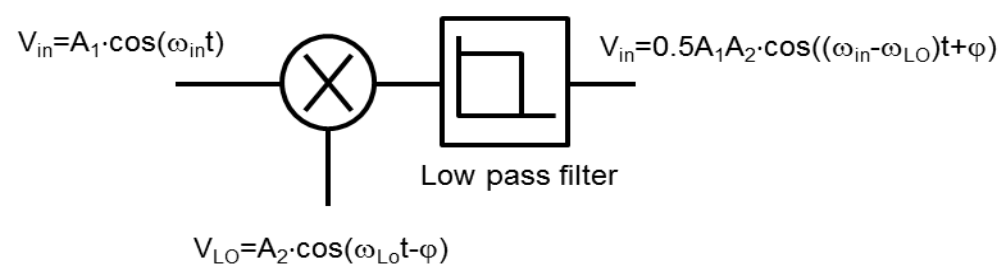

Fig. 2.20. Implementation of a phase shifter via a mixer and a filter

Fig. 2.21 shows the transfer function of an ideal phase shifter compared to an ideal time delay. At $\mathrm{f}_{0}$ the delay of both a phase shift and a time delay are the same and equal to: $\tau\left(f_{0}\right)=-\varphi_{0} /\left(2 \pi f_{0}\right)$. The amount of the delay in a limited band $[-\Delta f / 2, \Delta f / 2]$ around $f_{0}$ is approximated in eqn. 2.11. The delay produced by phase shifter is frequency dependent and therefore has a delay error with respect to the delay of a time delay block. 

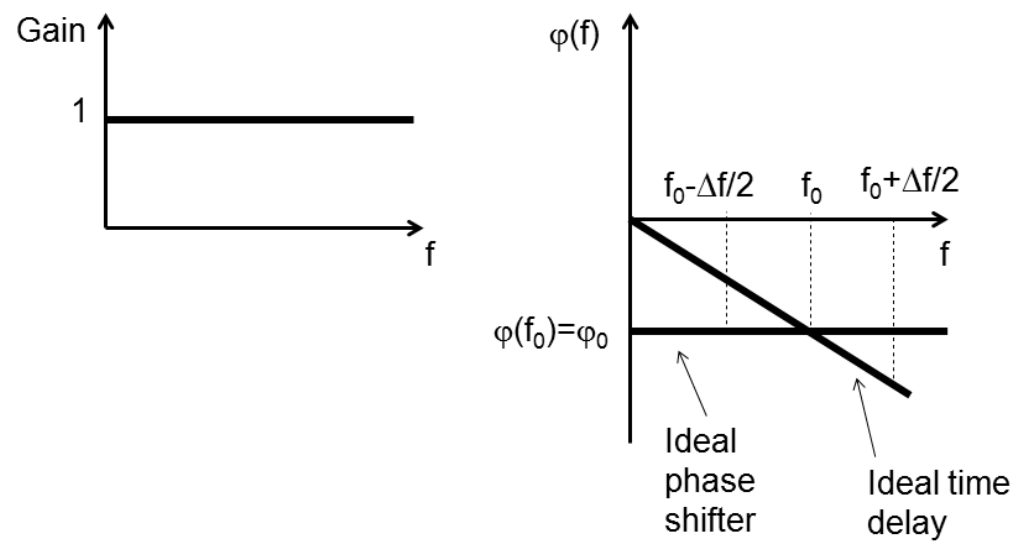

Fig. 2.21. Gain and phase transfer function of the phase shifter compared to time delay.

$\tau\left(f_{0} \pm \frac{\Delta f}{2}\right)=-\frac{\varphi\left(f_{0}\right)}{2 \pi\left(f_{0} \pm \frac{\Delta f}{2}\right)} \approx \tau\left(f_{0}\right)\left(1 \mp \frac{\Delta f}{2 f_{0}}\right)$

Instead of time delay blocks, phase shifters can be used in beamforming systems (Fig. 2.22). 


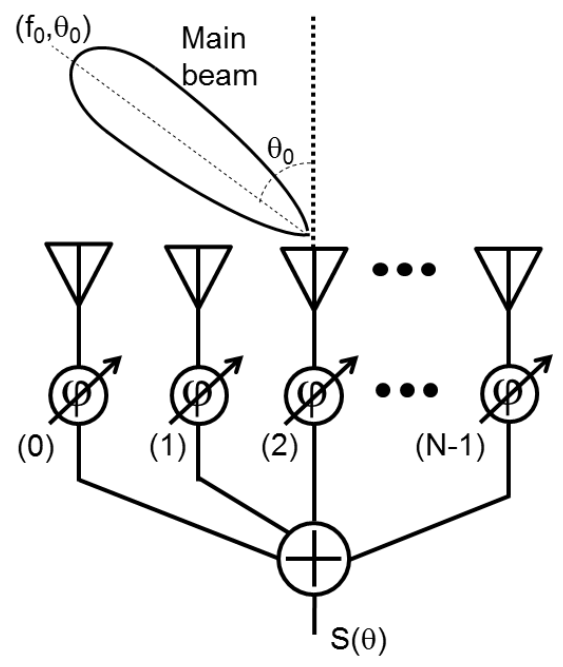

Fig. 2.22. phase shifter based phased array antenna system

The values of the phase shifters of the different antenna channels to direct the main beam toward $\theta_{0}$ are ( $f_{0}$ : operating frequency):

$$
\varphi_{k}=-2 \pi f_{0}(N-1-k) \frac{d}{c} \sin \theta_{0} \quad ; k=0, \ldots, N-1
$$

The implementation of a phased array antenna system via phase shifters can cause an unwanted phenomenon that is called beam squinting. It is the subject of the next section.

\subsection{Beam squinting}

When a phased array antenna system is implemented using phase shifters instead of time delays, the frequency-dependency in the delay produced by phase shifters causes the direction of the array beam to be frequency dependent (Fig. 2.23). This phenomenon is called beam squinting [1]. 


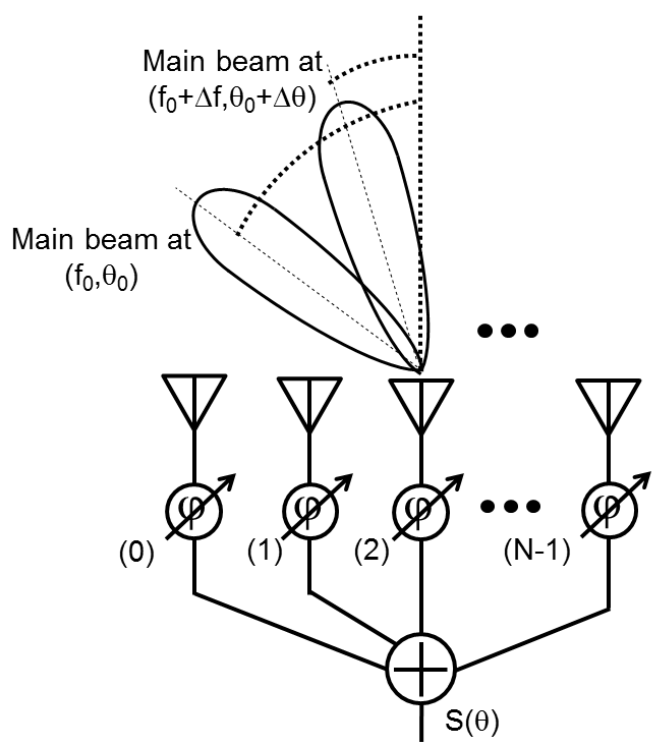

Fig. 2.23. Beam squinting in phase shifter based phased array systems

As an example, Fig. 2.24 shows beam squinting in the beam pattern of a 4 antenna element phased array antenna system implemented via phase shifters. In Fig. 2.24.a the operating frequency is at $f_{0}$, the interelement distances are equal to $0.5 \lambda_{0}$ and the phase shifters are tuned to direct the main beam toward $\theta=60^{\circ}$. Fig. 2.24.b and Fig. 2.24.c shows the occurrence of the shift in beam direction by changing the frequency to $\mathrm{f}=0.7 \mathrm{f}_{0}$ and $\mathrm{f}=1.3 \mathrm{f}_{0}$. Also for $\mathrm{f}=1.3 \mathrm{f}_{0}$ a grating lobe appears in the beam pattern. 
(a)

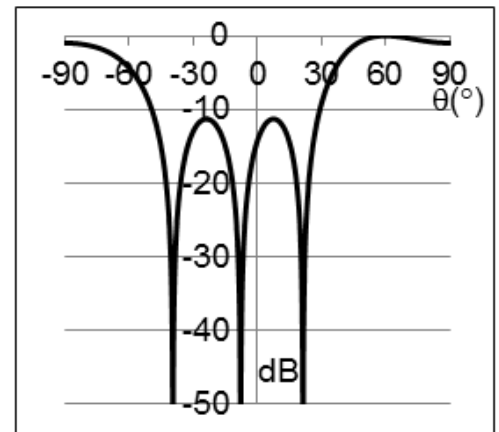

(b)

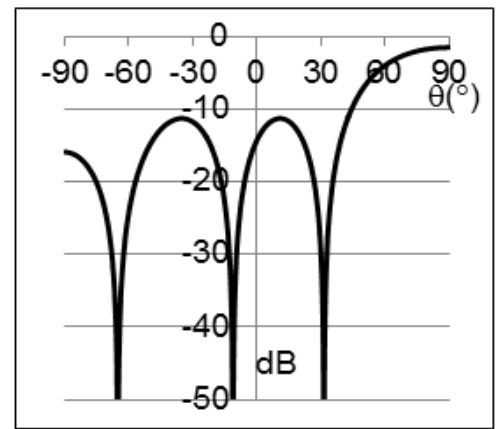

(c)

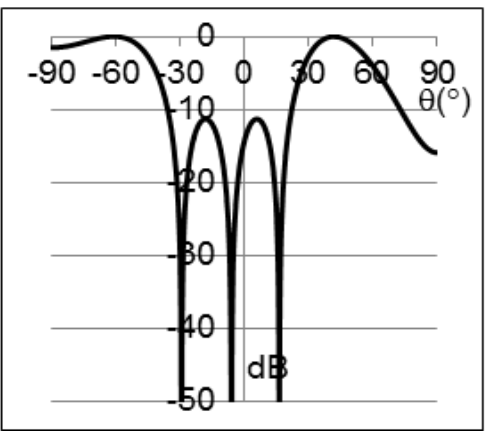

Fig. 2.24. The beam squinting phenomena in a 4 element phased array implemented via phased shifters $(a) f=f_{0},(b) f=0.7 f_{0},(c) f=1.3 f_{0}$ 
The amount of the beam squinting is estimated via eqn. 2.13 [1]

$\Delta \theta \approx-\tan \theta_{0} \frac{\Delta f}{f_{0}}$

The amount of beam squinting depends approximately linearly on the operating frequency change. For positive values of $\theta_{0}$, the signs of $\Delta \theta$ and $\Delta \mathrm{f}$ are reversed and for negative value of $\theta_{0}$, the signs of $\Delta \theta$ and $\Delta \mathrm{f}$ are the same. Beam squinting causes several unwanted effects: 1 ) Bandwidth limitation, 2) The reception of spatial interferences and 3) The rise of side lobes in hierarchical beamforming systems.

\subsubsection{Bandwidth limitation}

Suppose a phased array system implemented by phase shifters operates at $\mathrm{f}_{0}$, its main beam direction is toward $\theta_{0}$ and the gain of the main beam (not normalized) toward $\theta_{0}$ is equal to $G_{0}$. A frequency shift equal to $\Delta \mathrm{f}$ causes a direction shift equal to $\Delta \theta$ in the main beam, therefore, the gain of the shifted main beam at $\theta_{0}$ direction drops by $\Delta G$ (Fig. 2.25). More frequency shift results in more direction shift and consequently more gain drop in the main beam direction. The drop of the gain with respect to the frequency causes bandwidth limitations in the phased array antenna system. The amount of $\Delta f$ which corresponds to a $3 \mathrm{~dB}$ gain drop with respect to $\mathrm{G}_{0}$ defines the $3 \mathrm{db}$ bandwidth of the system.

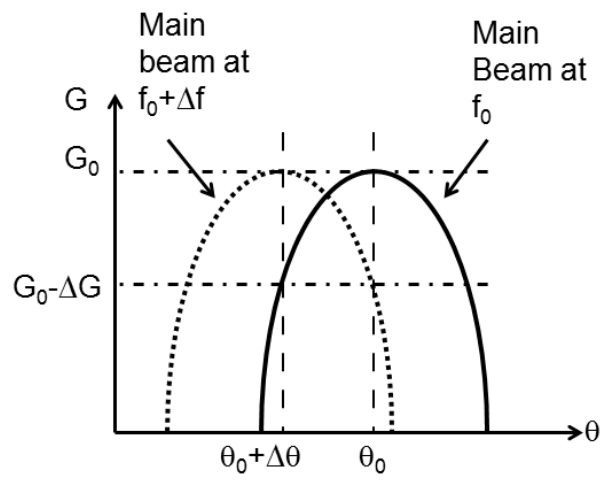

Fig. 2.25. Gain vs. frequency variations in a squinted beam 


\subsubsection{Degrading the spatial interference rejection}

Suppose the wanted signal is at frequency $\mathrm{f}_{0}$ and direction $\theta_{0}$ while the interference signal is at frequency $f_{0}+\Delta f$ and direction $\theta_{0}+\Delta \theta$ (Fig. 2.26). The wanted signal is received with a gain equal to $\mathrm{G}_{0}$. The aim of the phased array antenna system is to attenuate the interference signal by amount of $\Delta \mathrm{G}$ with respect to $\mathrm{G}_{0}$. But, because of the beam squinting, at $\mathrm{f}_{0}+\Delta \mathrm{f}$ the beam shifts toward the unwanted direction $\theta_{0}+\Delta \theta$, therefore, the spatial interference around $\left(f_{0}+\Delta f, \theta_{0}+\Delta \theta\right)$ is received with the gain $G_{0}$ which is not the aim of the phased array system and can cause degradation in the SNR of the received signal.

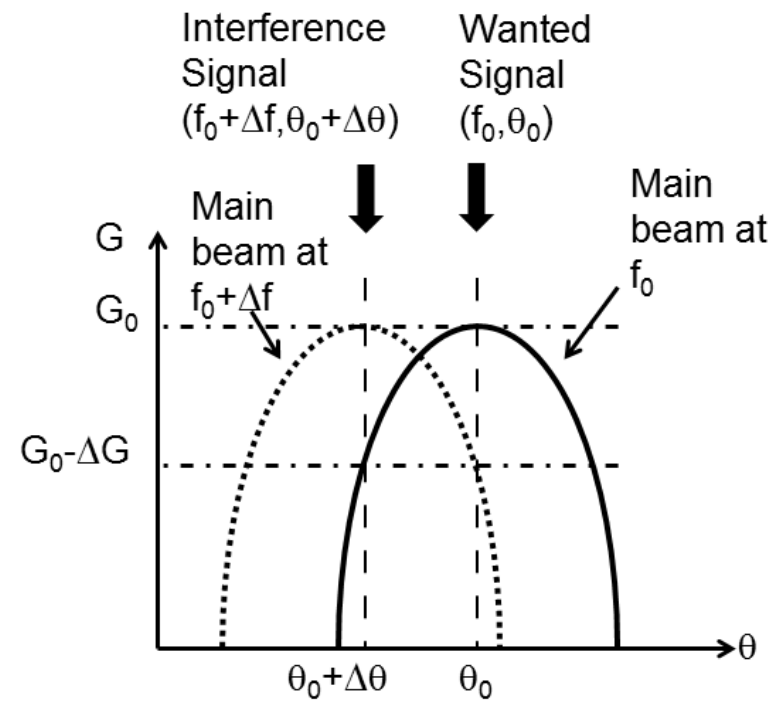

Fig. 2.26. The wanted and interference signal

\subsubsection{Increase of side lobe levels}

As Fig. 2.12 showed, in hierarchical beamforming systems with all layers implemented via time delay blocks, the grating lobes of the second layer of beamforming are cancelled by the nulls of the first layer. This keeps the side lobe levels limited. But, some kinds of 
wideband hierarchical beamforming systems are implemented with phase shifters at the first layer of hierarchy and with time delays at the next layers. The reason behind this type of design is that at RF frequencies the implementation of mixer-based phase shifters is simpler than the implementation of time delays and also mixer-based phase shifters down-convert the signals. The next layers are at IF frequencies and can be implemented via time delay blocks. The beam squinting of the first layer does not cause a significant shift on the direction of the total main beam. But, it causes unwanted side lobes in the total beam pattern, because the beam squinting causes the null positions of the first layer to not track, align and consequently cancel out the grating lobes of the second layer. This phenomenon makes large side-lobes and consequently degrades the spatial selectivity of the phased array for interferences. Fig. 2.27 shows a two layer, 16 antenna element hierarchical beamforming system with the first layer implemented with phase shifters and the second layer implemented with time delays. The inter-element distances $\left(d_{1}\right)$ are $0.5 \lambda_{0}$.

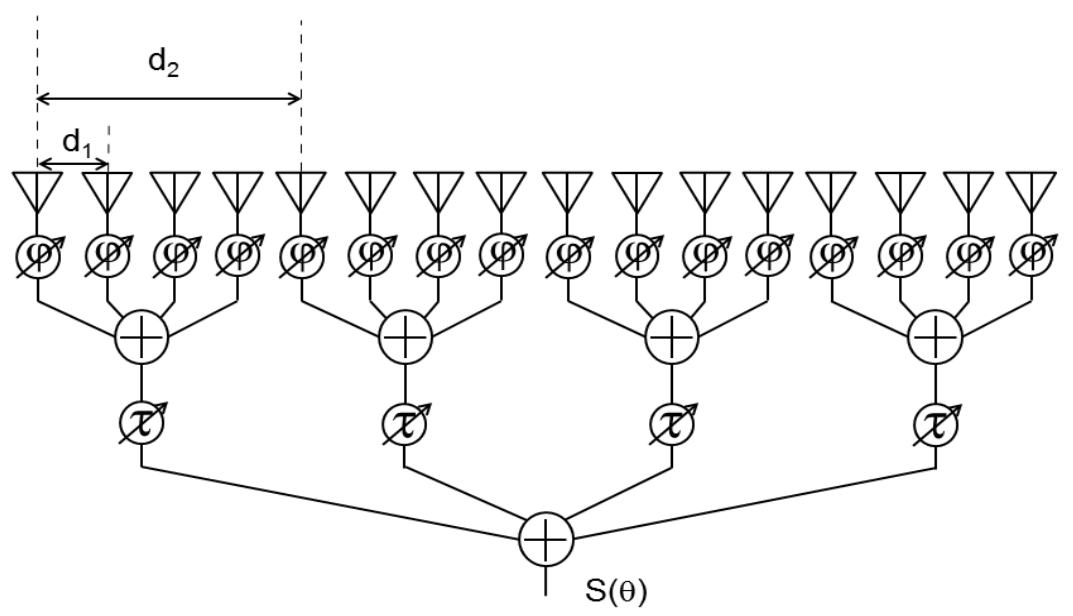

Fig. 2.27. A 16 element hierarchical array, having phase shifters in the first layer and time delays in the second layer.

Fig. 2.28.a shows the total beam pattern of the array at $f_{0}$ while the beam is toward $\theta=60^{\circ}$ and Fig. 2.28.b shows the beam pattern when frequency is $0.7 \mathrm{f}_{0}$. The side lobe levels at $0.7 \mathrm{f}_{0}$ dramatically increase. 
(a)

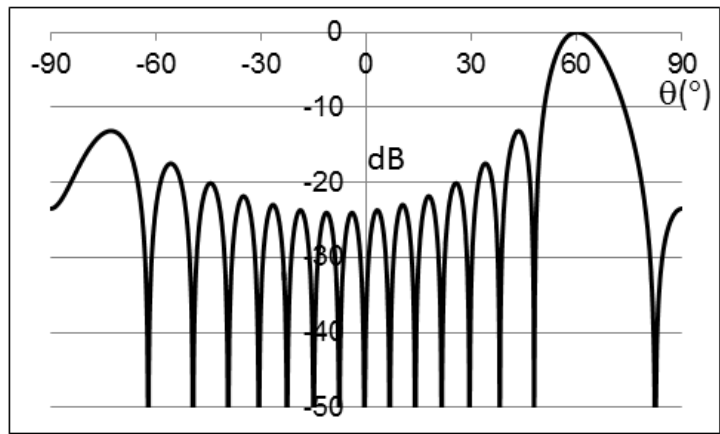

(b)

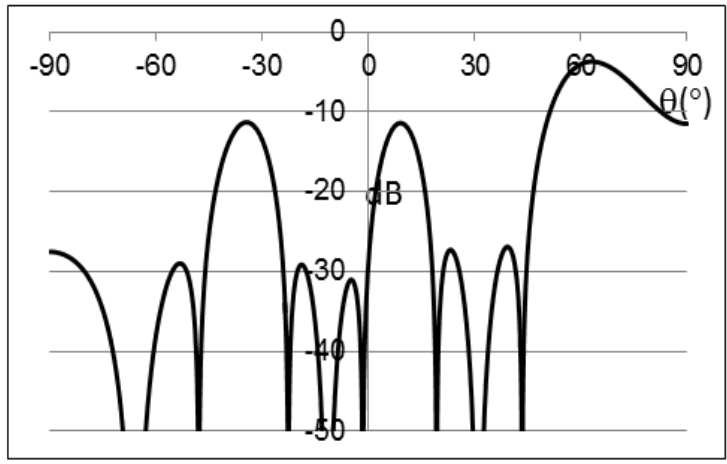

Fig. 2.28. The beam pattern of a hierarchical beamforming system $\left(1^{\text {st }}\right.$ layer phase shifters, $2^{\text {nd }}$ layer time delays) (a) $f=f_{0}$, (b) $f=0.7 f_{0}$

\subsection{Wideband beamforming}

If phase shifters are used in phased array antenna systems, the functional degradations originating from beam squinting can be avoided by keeping the operating bandwidth limited. In other words, phase shifters are a good solution for the implementation of narrow band phased array antenna systems. For the wideband phased array antenna systems, a solution to avoid beam squinting is the implementation of a system only with time delays. Using time delays guarantees that the direction of the beam remains independent to the frequency and no gain degradation in the requested spatial direction happens with respect to the frequency shift. 
A phased array antenna system implemented via time delay blocks is much less vulnerable to spatial interferences compared to the phase shifter based scenario. Fig. 2.29 shows the received wanted signal and interference for two possible scenarios, implemented via phase shifters and implementation via time delays. The spatial interference rejection of the time delay based scenario does not suffer from beam squinting. Of course by decreasing the frequency, the amount of $d / \lambda$ narrows and widens the beam width for both scenarios, which consequently degrades the spatial selectivity. 
(a)

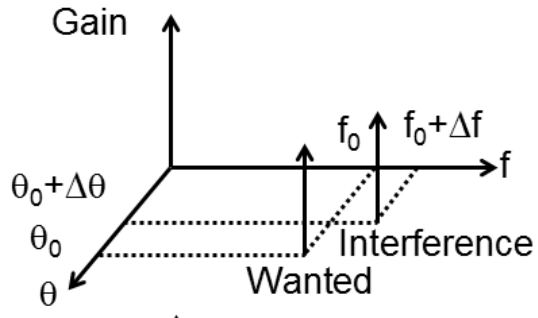

(b)

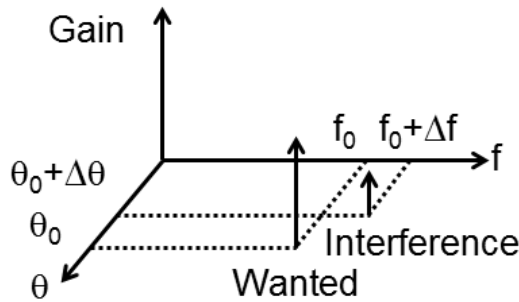

(c)

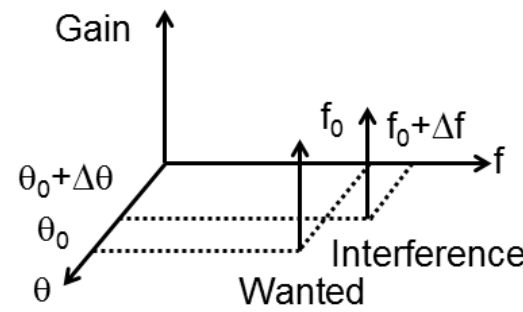

Fig. 2.29. The signal and interference relation for (a) radiated signal density (b) time delay based scenario and (c) phase shifter based scenario

In hierarchical beamforming, the implementation of all layers via time delays keeps the side lobe levels low, because, nulls of the first layer track and cancel out the grating lobes of the second layer, independent of the frequency shift. As an example, Fig. 2.30 shows the beam pattern of the 16 element, 2 layer hierarchical beamforming implemented system with only time delays (refer to Fig. 2.11). The inter-element distances are equal to $0.5 \lambda_{0}$. Fig. 2.30.a shows the beam pattern of the total array at $\mathrm{f}=\mathrm{f}_{0}$ and Fig. 2.30.b shows the beam pattern of the same array at $\mathrm{f}=0.7 \mathrm{f}_{0}$. Compared to the graphs of Fig. 2.28, Fig. 2.30 shows that by shifting the frequency no extra side lobes occur in a time delay based hierarchical phased array antenna system. 
(a)

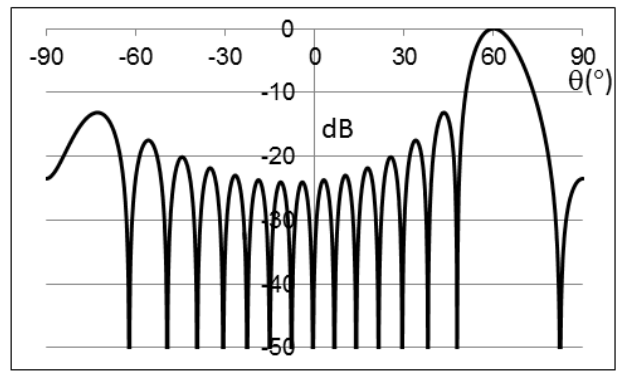

(b)

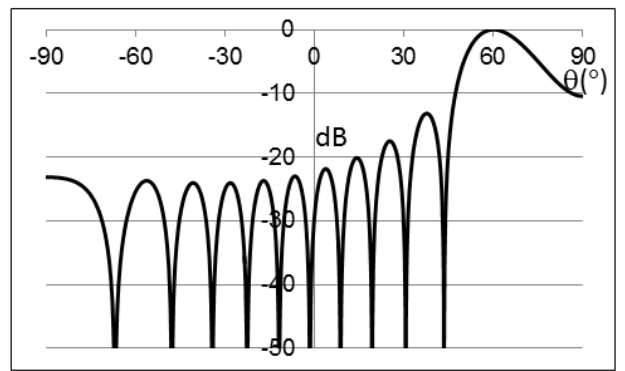

Fig. 2.30. The beam pattern of a 16 element hierarchical phased array antenna system implemented via time delays at $(a) f=f_{0}(b) f=0.7 f_{0}$

\subsection{Conclusion}

The structure of the phased array antenna system as a method to produce re-configurable beam patterns is analyzed. In addition, the operational mechanism of the linear phased array antenna system as a basic building block of all other spatially arranged phased arrays is explained. The array factor of the linear phased array was established and important characteristics of the array factor were explained and discussed, including beam direction, beam width, null directions, side lobe levels/directions and grating lobes. The design parameters of the array were explained and their effects on the array factor were shown. Furthermore, the hierarchical phased array antenna systems and their properties were shown. 
It was shown that tuning the time delay blocks in the phased array antenna system steers the beam and null directions. Different methods for physical implementation of the time delay blocks were reviewed and compared to each other. It was shown that the implementation methods are limited by the maximum and minimum achievable delay, operating frequency, noise, size and cost. Compared to other mentioned methods the $\mathrm{g}_{\mathrm{m}}-(\mathrm{R}) \mathrm{C}$ filters seem good candidates for many applications because they work at several $\mathrm{GHz}$ frequencies while having small size and cost.

The use of phase shifters as an alternative method for time delays has been explained. It was shown that phase shifters can imitate the transfer function of a time delay block in limited frequency bandwidths. However, using phase shifters causes the beam squinting phenomenon which limits the bandwidth of the phased array and makes the phased array antenna receiver more vulnerable to spatial interferences. Therefore, implementation of time delay blocks in phased array antenna systems is a solution having wide bandwidths combined with high spatial selectivity.

\section{References}

[1] R. J. Mailloux, "Phased Arrays in Radar and Communication Systems," in Phased Array Antenna Handbook, 2nd ed., Norwood, Artech house, 2005.

[2] N. Fourikis, "Advanced Array Systems, Applications and RF Technologies," Academic Press, 2000.

[3] H. J. Visser, Array and Phased Array Antenna Basics, Wiley, 2006.

[4] G. B. Arfken, H. J. Weber and F. E. Harris, in Mathematical Methods for Physicists, Sixth Edition: A Comprehensive Guide, Academic Press; 6 edition, 2005. 
[5] M. J. M. Pelgrom, in Analog-to-Digital Conversion, Springer; 2nd ed. , 2013.

[6] R. J. van de Plassche, "CMOS Integrated Analog-to-Digital and Digital-to-Analog Converters," Springer; 2nd edition, 2013, May.

[7] L. Zhuang, A. Meijerink, C. Roeloffzen, D. Marpaung and W. van Etten, "Novel ring resonator-based optical beamformer for broadband phased array receive antennas," IEEE Lasers and Electro-Optics Society, 2008. LEOS 2008. 21st Annual Meeting of the, pp. 20-21, 9-13 November 2008.

[8] G. Gonzalez, "Microwave Transistor Amplifiers: Analysis and Design," Prentice Hall; 2 edition, 1996, August.

[9] T. Chu, J. Roderick and H. Hashemi, "An Integrated UltraWideband Timed Array Receiver in $0.13 \mu \mathrm{m}$ CMOS Using a PathSharing True Time Delay Architecture," IEEE J.Solid-State Circuits, vol.42, no. 12, pp. 2834- 2850, Dec. 2007.

[10] P. E. Allen and D. R. Holberg, "CMOS Analog Circuit Design," Oxford Series in Electrical and Computer Engineering, 2011, August.

[11] A. V. Oppenheim, R. W. Schafer and J. Buck, "Filter Design Techniques," in Discrete-Time Signal Processing, 2nd ed., New Jersey, Pretince-Hall, 1999.

[12] F. Ellinger, "Passive Devices and Networks," in Radio Frequency Integrated Circuits and Technologies, Berlin, Germany, Springer, 2007.

[13] T. H. Lee, "noise," in The Design of CMOS Radio-Frequency Integrated Circuits, 2nd ed., Cambridge University Press, 2003.

[14] S. Pavan and Y. Tsividis, "High Frequency Continuous Time 
Filters in Digital CMOS," Springer, 2000, January.

[15] J. Litva, "Digital Beamforming in Wireless Communications," Artech House, 1996, August. 


\section{Chapter 3 \\ A Quality Criterion for Delay Variations versus Frequency ${ }^{1}$}

\subsection{Introduction}

Time delay circuits have wide applications in different systems, such as beamforming systems, delay locked loops, filters and equalizers. In some of these applications it is important to maintain constant delay over a significant frequency band, e.g. for wideband beamforming systems ("phased array antenna systems") [1]. This is because delay variations result in changes of the beam pattern, such as in beam direction and side lobe levels [1]. As practical delay circuits show some delay variation over frequency, characterizing this variation over the relevant band is important. The quality of time delay circuits is often evaluated in terms of group delay. In this chapter it is shown that the group delay is useful but not sufficient to characterize delay variation vs. frequency. Therefore, a criterion is proposed that, together with group delay relates the delay variation and frequency bandwidth to each other. Fig. 3.1 shows the phase vs. frequency

\footnotetext{
${ }^{1}$ Except for section 3.5 which has been extended, this chapter is a copy of the following paper:

S. K. Garakoui, E. A. M. Klumperink, B. Nauta and F. E. van Vliet, "Time delay circuits: A quality criterion for delay variations versus frequency," IEEE ISCAS Proc., 2010, pp. 4281-4284.
} 
characteristic of an ideal phase shifter and an ideal time delay block with time delay equal to $\tau_{\mathrm{d}}$ (In this chapter the frequency and phase axes are linear).

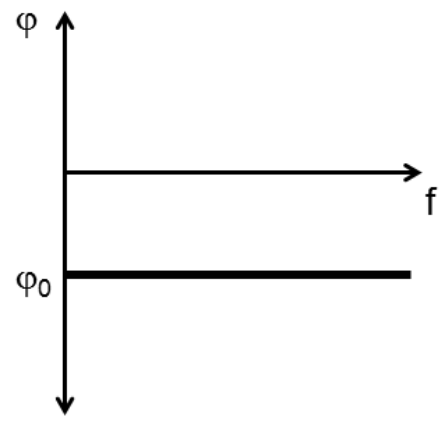

(a)

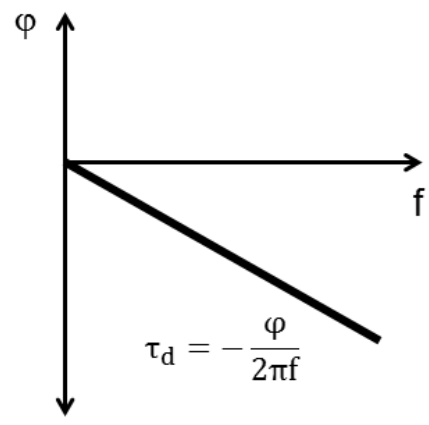

(b)

Fig. 3.1. Phase vs. frequency for (a) an ideal phase shifter and (b) an ideal time delay element

Both an ideal phase shifter and ideal delay block should have unity gain, but they have different group delays versus frequency. Group delay $(\tau \mathrm{g})$ is defined as follows [2]:

$$
\tau_{g}=-\frac{1}{2 \pi} \frac{d \varphi}{d f}
$$

For an ideal phase shifter, the group delay vs. frequency is constant and equal to zero. For an ideal time delay block it is also constant but non-zero and with a group delay equal to the amount of time delay (see Fig. 3.1). For a phase shifter block the conditions of zero group delay and unity amplitude gain vs. frequency defines the necessary and sufficient conditions to define the block as an ideal phase shifter. However, the condition of constant group delay and unity amplitude gain vs. frequency does not necessarily correspond to constant time delay. Fig. 3.2.a shows two phase vs. frequency plots which have identical and constant group delay for the frequency range $\left[f_{0}-\Delta f\right.$, 
$\left.\mathrm{f}_{0}+\Delta \mathrm{f}\right]$. However, only line 1 crosses through the point $(\mathrm{f}=0, \varphi=0)$ which defines the characteristic of an ideal time delay block.

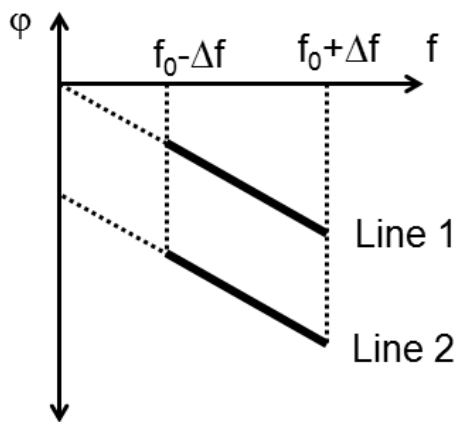

(a)

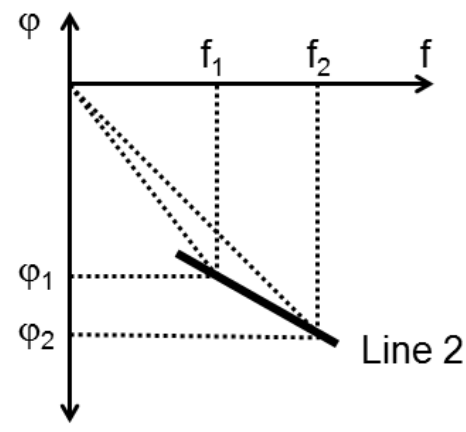

(b)

Fig. 3.2. (a) two phase lines with equal group delay where only Line 1 defines an ideal time delay (b) Different points on Line 2 corresponding to different time delays

For Line 2 the time delay varies with frequency. Fig 3.2.b illustrates this for two example frequencies $f_{1}$ and $f_{2}$, where the time delays are equal to $\tau_{1}=-\varphi_{1} / 2 \pi f_{1}$ and $\tau_{2}=-\varphi_{2} / 2 \pi f_{2}$ respectively. So the group delay is the same, but the time delay is not, which shows that knowledge about group delay is not sufficient to quantify the amount of time delay versus frequency [3],[4]. In section 3.2 a new criterion $f_{\varphi=0}$ is proposed that relates the amount of time delay variation to the frequency range. In section 3.3 the amount of time delay variation for circuits with a frequency dependent group delay is analyzed. Section 3.4 gives examples, and derives $f_{\varphi=0}$ equations for two commonly used time delay circuits: an RC and an LC delay circuit. In section $3.5 \mathrm{f}_{\varphi=0}$ is extracted for a cascade of time delay blocks. In section 3.6 the ability of $f_{\varphi=0}$ for estimation of the delay versus frequency variations is verified via simulations.

\subsection{A new criterion $\left(f_{\varphi=0}\right)$ for time delay circuits}

Fig. 3.3.a gives an example for the phase vs. frequency characteristic of a typical time delay block (e.g. an RC delay circuit). If 
in the frequency range $\left[\mathrm{f}_{0}-\Delta \mathrm{f}, \mathrm{f}_{0}+\Delta \mathrm{f}\right]$ the transfer function is approximated by a straight line, then two parameters are sufficient to fully characterize this line:

1. The slope of the phase versus frequency, i.e. $-\tau_{\mathrm{g}}$

2. The phase for at least one frequency point

This point can be any point of the line in the relevant frequency band or any point on the linearly extrapolated phase line. We propose to use the point with zero phase where the line crosses the frequency axis and we will call this frequency $\mathrm{f}_{\varphi=0}$. It will be shown later (in section 3.5) that this point has a unique property: in a cascaded line of several identical delay blocks, the $f_{\varphi=0}$ of the total line remains equal to the $\mathrm{f}_{\varphi=0}$ of the individual delay blocks. We introduce $\mathrm{f}_{\varphi=0}$ as a new criterion to approximately quantify time delay variations inside the frequency band. The criterion has some interesting characteristics:

1. $\mathrm{f}_{\varphi=0}=0$ corresponds to ideal (constant) time delay behavior inside the band

2. $\mathrm{f}_{\varphi=0}= \pm \infty$ corresponds to ideal (constant) phase shift behavior inside the band.

3. $\mathrm{f}_{\varphi=0} \neq 0$ in combination with constant group delay corresponds to approximate time delay behavior, where the delay variation versus frequency can be predicted accurately.

Fig. 3.3.b shows two transfer function examples with constant group delay inside the band $\left[\mathrm{f}_{0}-\Delta \mathrm{f}, \mathrm{f}_{0}+\Delta \mathrm{f}\right]$ but with different $\mathrm{f}_{\varphi=0} \neq 0$. 


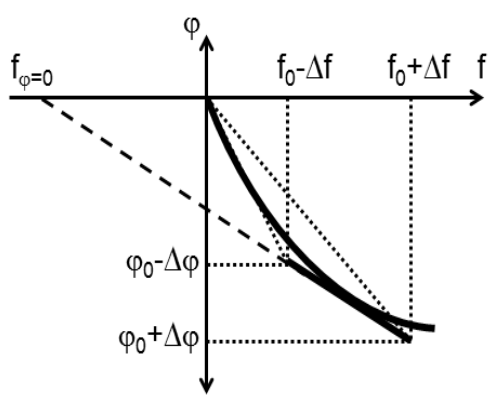

(a)

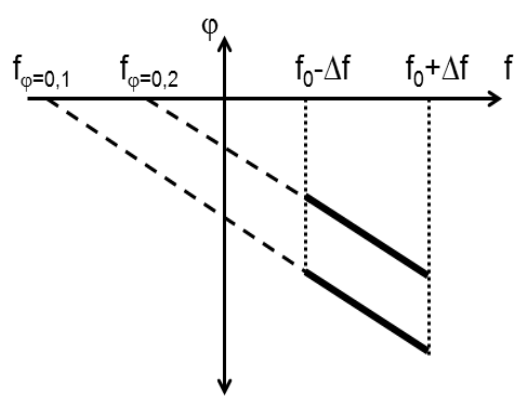

(b)

Fig. 3.3. (a) $\varphi$ vs. $f$ for a frequency range which the curve is modeled as a straight line inside it .(b) $\mathrm{f}_{\varphi=0}$ can be used as a criterion to differ $\varphi$ vs. f lines with equal group delays

Now the time delay variation for these time delay block approximations is related to the center frequency of the band $\left(\mathrm{f}_{0}\right)$, the frequency range $( \pm \Delta f)$, the group delay $\left(\tau_{\mathrm{g}}\right)$ and the proposed criterion $\left(\mathrm{f}_{\varphi=0}\right)$ which is shown in Fig. 3.4. The amount of time delay at $\mathrm{f}_{0}$ is $\varphi\left(\mathrm{f}_{0}\right) / 2 \pi \mathrm{f}_{0}$, whereas it is $-\varphi\left(\mathrm{f}_{0}+\Delta \mathrm{f}\right) / 2 \pi\left(\mathrm{f}_{0}+\Delta \mathrm{f}\right)$ at $\mathrm{f}_{0}+\Delta \mathrm{f}$, and $-\varphi\left(\mathrm{f}_{0}-\right.$ $\Delta f) / 2 \pi\left(f_{0}-\Delta f\right)$ at $f_{0}-\Delta f$. Eqn. 3.2 defines the phase versus frequency, depending on group delay $\left(\tau_{\mathrm{g}}\right)$ and $\mathrm{f}_{\varphi=0}$. Eqn. 3.3 and eqn. 3.4 show time delays at frequencies $f_{0}$ and $f_{0}+\Delta f$. The delay at $f_{0}-\Delta f$ can be found from eqn. 3.2. However, because the $\varphi$ vs. $f$ transfer function of Fig. 3.4 is odd symmetric around $f_{0}$ the absolute delay variation from $f_{0}-\Delta f$ to $f_{0}$ is equal to delay variation from $\mathrm{f}_{0}$ to $\mathrm{f}_{0}+\Delta \mathrm{f}$. Because of this reason, we solve the equations only for upper half part of the frequency. 


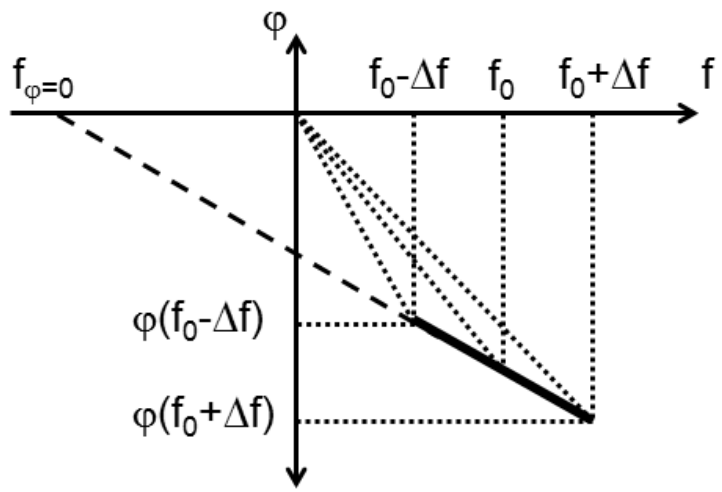

Fig. 3.4. Illustration of a phase characteristic of a delay block which has constant group delay inside $\left[\mathrm{f}_{0}-\Delta \mathrm{f}, \mathrm{f}_{0}+\Delta \mathrm{f}\right]$

$\varphi(f)=-\tau_{g}\left(f_{0}\right) \cdot 2 \pi\left(f-f_{\varphi=0}\right)$

$\tau_{d}\left(f_{0}\right)=-\frac{\varphi\left(f_{0}\right)}{2 \pi f_{0}}=\tau_{g}\left(f_{0}\right)\left(1-\frac{f_{\varphi=0}}{f_{0}}\right)$

$\tau_{d}\left(f_{0}+\Delta f\right)=-\frac{\varphi\left(f_{0}+\Delta f\right)}{2 \pi\left(f_{0}+\Delta f\right)}=\tau_{g}\left(f_{0}\right)\left(1-\frac{f_{\varphi=0}}{f_{0}+\Delta f}\right)$

The ratio of the delay variation inside the band to the absolute value of the delay at $f_{0}$ is useful to quantify the relative deviation of the delay that we can get inside the band (sometimes referred to as delay error or delay accuracy). Eqn. 3.5 shows this ratio and also an approximation for the condition $\Delta \mathrm{f} / \mathrm{f}_{0}<<1$. Because group delay is considered constant in the frequency range, eqn. 3.5 is independent of the group delay.

$$
\frac{\tau_{d}\left(f_{0}+\Delta f\right)-\tau_{d}\left(f_{0}\right)}{\tau_{d}\left(f_{0}\right)}=\frac{\frac{f_{\varphi=0}}{f_{0}}-\frac{f_{\varphi=0}}{f_{0}+\Delta f}}{1-\frac{f_{\varphi=0}}{f_{0}}} \approx \frac{\frac{f_{\varphi=0}}{f_{0}}}{1-\frac{f_{\varphi=0}}{f_{0}}} \frac{\Delta f}{f_{0}}
$$


For a block with constant phase in the frequency band, $\mathrm{f}_{\varphi=0}$ becomes infinite and with condition $\Delta \mathrm{f} / \mathrm{f}_{0} \ll 1$ eqn. 3.5 will result in eqn. 3.6:

$$
\left.\frac{\tau_{d}\left(f_{0}+\Delta f\right)-\tau_{d}\left(f_{0}\right)}{\tau_{d}\left(f_{0}\right)}\right|_{\text {phase-shifter }} \approx-\frac{\Delta f}{f_{0}}
$$

For an ideal time delay, $\mathrm{f}_{\varphi=0}$ is equal to zero and eqn. 3.5 will result in:

$$
\left.\frac{\tau_{d}\left(f_{0}+\Delta f\right)-\tau_{d}\left(f_{0}\right)}{\tau_{d}\left(f_{0}\right)}\right|_{\text {Time-delay }} \approx 0
$$

In summary, if $\mathrm{f}_{\varphi=0}$ is non-zero and the group delay is constant for a circuit, it can be used as an approximation of a time delay circuit. Eqn. 3.5 gives the relative variation of the time delay over the band. In the next section we derive the time delay variation for more realistic time delay blocks for which the group delay is frequency dependent.

\subsection{Time delay of blocks with frequency dependent group delay}

Fig. 3.5 shows $\varphi$ vs. $f$ characteristic of a typical practical delay circuit with a group delay that is frequency dependent. If we know the group delay of the characteristic at the center frequency $f_{0}$ of the band, we can again derive an estimate of the delay for $f_{0}+\Delta f$. 


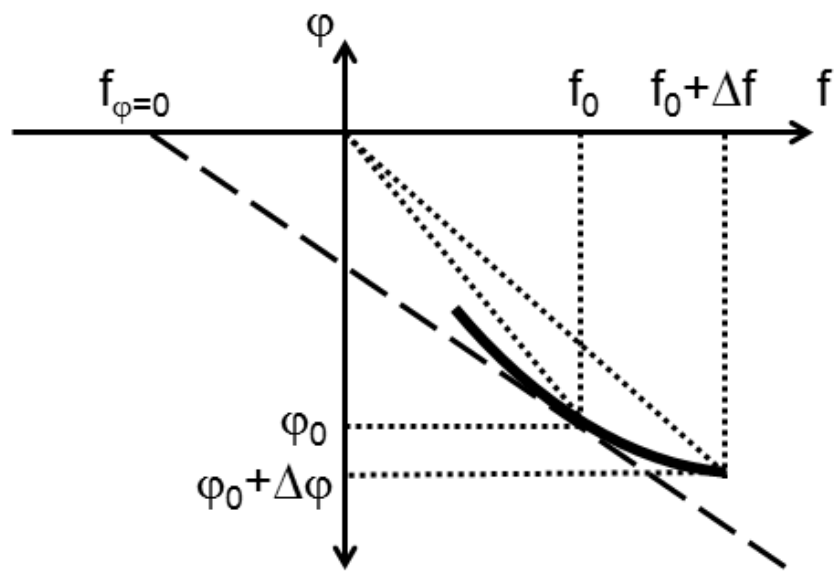

Fig. 3.5. Phase vs. frequency transfer function with a frequency dependent group delay

To this end we draw the slope-line at the point $\left(\mathrm{f}_{0}, \varphi\left(\mathrm{f}_{0}\right)\right)$. This line crosses the $f$-axis at point $f_{\varphi=0}$, and hence the following equation holds:

$\left.\frac{\partial \varphi}{\partial f}\right|_{f_{0}}=\frac{\varphi_{0}}{f_{0}-f_{\varphi=0}}$

If inside the band, the variation of the phase around $\mathrm{f}_{0}$ is not very large, we can approximate it with the first two terms of a Taylor series. In this case the calculations are identical to those in the previous section and the delay at $\mathrm{f}_{0}+\Delta \mathrm{f}$ can be estimated with eqn. 3.4, while the relative delay variation can be assessed by eqn. 3.5. If desired, of course a higher order Taylor series also can be used. The effect of the curvature in the phase characteristic on delay as shown by equations 3.9 and 3.10 : 
$\tau_{d}\left(f_{0}+\Delta f\right)=-\frac{\varphi\left(f_{0}+\Delta f\right)}{2 \pi\left(f_{0}+\Delta f\right)}$

$\approx-\frac{\varphi\left(f_{0}\right)}{2 \pi\left(f_{0}+\Delta f\right)}+\frac{\tau_{g}\left(f_{0}\right)}{\left(f_{0}+\Delta f\right)} \cdot \Delta f+\frac{1}{2} \frac{\left.\frac{\partial \tau_{g}}{\partial f}\right|_{f_{0}}}{\left(f_{0}+\Delta f\right)} .(\Delta f)^{2}$

If $\Delta \mathrm{f} / \mathrm{f}_{0}<<1$ then this equation can be approximated as:

$\tau_{d}\left(f_{0}+\Delta f\right) \approx-\frac{\varphi\left(f_{0}\right)}{2 \pi\left(f_{0}\right)}\left(1-\frac{\Delta f}{f_{0}}\right)+\tau_{d}\left(f_{0}\right) \cdot \frac{\Delta f}{f_{0}}+\left.\frac{1}{2} \cdot f_{0} \cdot \frac{\partial \tau_{d}}{\partial f}\right|_{f_{0}} \cdot\left(\frac{\Delta f}{f_{0}}\right)^{2}$

However, for finding time delay, in many cases a linear approximation of the phase vs. frequency curve is enough and there is no need to use the second order term of the eqn. 3.10. In the next section we explain how to find $f_{\varphi=0}$ at any point of the phase vs. frequency curve.

\section{4 $\mathbf{f}_{\varphi=0}$ for an RC and LC circuit}

There are different types of time delay circuits and based on their structure they show different phase-frequency transfer functions. We will now solve eqn. 3.8 for two commonly used examples of delay blocks, an RC delay block and an LC segment of an infinite LC delay line [5]. If we assume that the operating frequency of an LC delay line is much less than $(2 \pi)^{-1}(L C)^{-0.5}$, the load impedance which every segment sees at its output is real and equal to $Z_{0}=(L / C)^{0.5}$, (the "characteristic impedance"). Fig. 3.6 shows an RC delay block and one segment of an LC delay line and their phase vs. frequency transfer functions. 


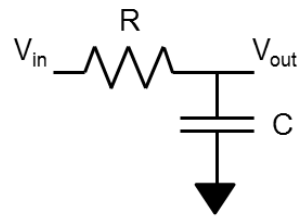

$\varphi=-\arctan (2 \pi R C f)$

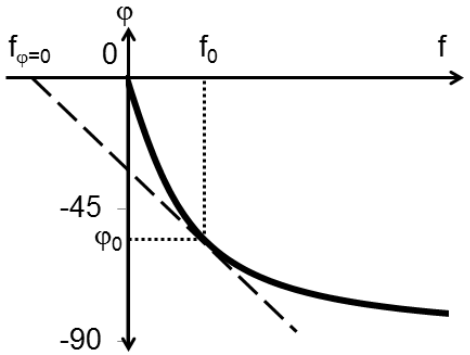

(a)
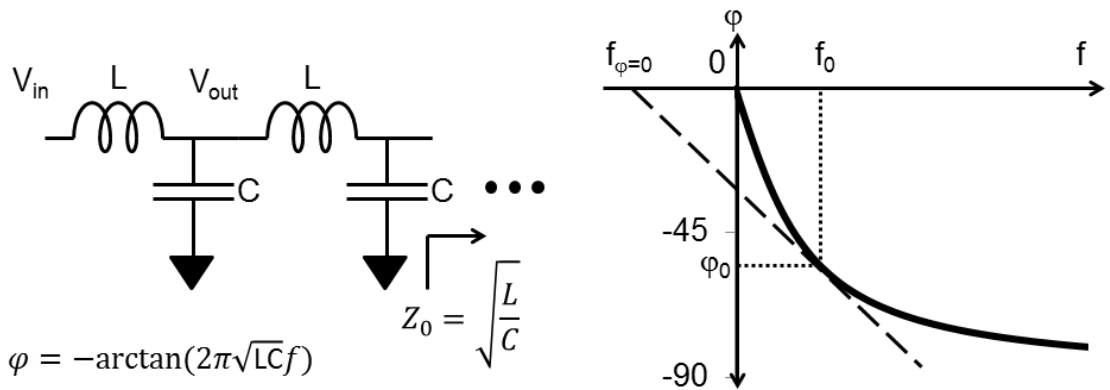

(b)

Fig. 3.6. Phase vs. frequency transfer function for (a) an RC delay block and (b) one segment of an infinite LC delay line

By substituting phase vs. frequency characteristics of each block of Fig. 3.6 into eqn. 3.8 the values of $\mathrm{f}_{\varphi=0}$ can be found. This substitution results in eqn. 3.11.a for an RC delay block and 3.11.b for an LC delay segment.

$$
\begin{gathered}
\left.\frac{\partial}{\partial f}(-\arctan (2 \pi R C f))\right|_{f_{0}}=-\frac{\arctan \left(2 \pi R C f_{0}\right)}{f_{0}-f_{\varphi=0}} \\
\left.\frac{\partial}{\partial f}(-\arctan (2 \pi \sqrt{L C} f))\right|_{f_{0}}=-\frac{\arctan \left(2 \pi \sqrt{L C} f_{0}\right)}{f_{0}-f_{\varphi=0}}
\end{gathered}
$$



is:

For an RC delay block the solution of eqn. 3.11.a in terms of the $f_{\varphi=0}$

$f_{\varphi=0}=f_{0}\left[1-\frac{1+\left(2 \pi R C f_{0}\right)^{2}}{2 \pi R C f_{0}} \cdot \arctan \left(2 \pi R C f_{0}\right)\right]$

If $\left(2 \pi R C f_{0}\right)<<1$, this equation simplifies to:

$f_{\varphi=0}=-f_{0} \cdot\left(2 \pi R C f_{0}\right)^{2}=-f_{0} \cdot\left(\frac{f_{0}}{f_{-3 d B}}\right)^{2}$

These equations show that the amount of $\mathrm{f}_{\varphi=0}$ increases for higher center frequency, leading to more delay variation according to eqn. 3.5, which fits to the expectation. Similarly, eqn. 3.13.a is the result of $\mathrm{f}_{\varphi=0}$ for an LC delay segment and for $2 \pi(L C)^{0.5} f_{0}<<1$, it reduces to eqn. 3.13.b.

$f_{\varphi=0}=f_{0}\left[1-\frac{1+\left(2 \pi \sqrt{L C} f_{0}\right)^{2}}{2 \pi \sqrt{L C} f_{0}} \cdot \arctan \left(2 \pi \sqrt{L C} f_{0}\right)\right]$

$f_{\varphi=0}=-f_{0} \cdot\left(2 \pi \sqrt{L C} f_{0}\right)^{2}$

Again, we see an increase of the $f_{\varphi=0}$ for higher center frequency.

\section{$3.5 \mathbf{f}_{\varphi=0}$ of the cascaded delay}

Fig. 3.7 shows the phase vs. frequency (phase transfer function) of two different delay blocks for the range between $f_{0}$ and $f_{0}+\Delta f$. They have different group delay and $\mathrm{f}_{\varphi=0}$. Cascading them with buffers in between which omits the loading effect between them, or cascading them without buffer but with considering the loading effect of the next stage on the previous one results in a new delay block. 

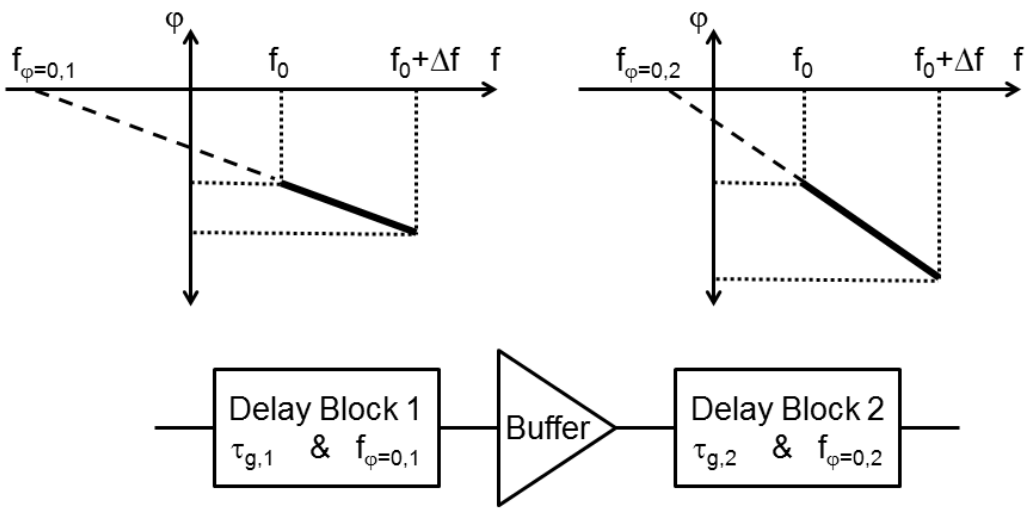

Fig. 3.7. Cascading delay blocks

The phase transfer function of the total cascade is the result of the summation of the phase transfer function of each individual block. Eqn. 3.14.a and eqn. 3.14.b are the phase transfer functions of individual blocks and eqn. 3.15 is the phase transfer function of the total cascade.

$\varphi_{1}=\tau_{g 1}\left(f-f_{\varphi=0,1}\right)$

$\varphi_{2}=\tau_{g 2}\left(f-f_{\varphi=0,2}\right)$

$\varphi=\left(\tau_{g 1}+\tau_{g 2}\right) f-\left(\tau_{g 1} f_{\varphi=0,1}+\tau_{g 2} f_{\varphi=0,2}\right)$

The total group-delay ( $\tau_{g \text {,cascadde }}$ ) and $\mathrm{f}_{\varphi=0 \text {,cascade }}$ of the cascade is extracted from the phase transfer function of the total cascade. The group delay of the cascade is equal to the sum of the group delays of individual blocks and $\mathrm{f}_{\varphi=0 \text {,cascaded }}$ is written in eqn. 3.16.

$f_{\varphi=0, \text { cascade }}=f_{\varphi=0,1} \cdot \frac{\tau_{g, 1}}{\tau_{g, 1}+\tau_{g, 2}}+f_{\varphi=0,2} \cdot \frac{\tau_{g, 2}}{\tau_{g, 1}+\tau_{g, 2}}$ 
This method can be extended to a cascade of several (n) delay blocks. The total group delay is the sum of the group delay of individual delay blocks and $\mathrm{f}_{\varphi=0, \text { cascade }}$ is written in eqn. 3.17.

$$
\begin{aligned}
f_{\varphi=0, \text { cascade }}=f_{\varphi=0,1} & \frac{\tau_{g, 1}}{\tau_{g, 1}+\tau_{g, 2}+\cdots+\tau_{g, n}} \\
& +f_{\varphi=0,2} \cdot \frac{\tau_{g, 2}}{\tau_{g, 1}+\tau_{g, 2}+\cdots+\tau_{g, n}}+\cdots \\
& +f_{\varphi=0, n} \cdot \frac{\tau_{g, n}}{\tau_{g, 1}+\tau_{g, 2}+\cdots+\tau_{g, n}}
\end{aligned}
$$

Eqn. 3.17 shows that if all delay blocks are identical, then $\mathrm{f}_{\varphi=0 \text {,cascade }}$ will be equal to that of each individual delay block. Also, for the LC delay line in Fig. 3.6.a, in which the loading effect is already taken into account, the $\mathrm{f}_{\varphi=0}$ of the whole delay line is equal to that of each LC segment.

\subsection{Simulation results}

To examine the ability of the proposed criterion $\mathrm{f}_{\varphi=0}$ to predict time delay variations over a frequency band, we continue with two examples of delay circuits: 1) an RC delay block and 2) an LC delay line. Suppose that both of them are designed for $\mathrm{f}_{0}=1 \mathrm{GHz}$ and we want to evaluate their time delay variation at $\pm 100 \mathrm{MHz}$ around $\mathrm{f}_{0}$. For both of the examples we use linear approximation of the phase vs. frequency transfer function and we will show that it results in a reasonable approximation of the circuit behavior.

Example 1: An RC time delay block (Fig. 3.6a), with $\mathrm{R}=1 \mathrm{~K} \Omega$, $\mathrm{C}=490 \mathrm{fF}$, at $\mathrm{f}_{0}=1 \mathrm{GHz}$ has a time delay equal to $\tau_{\mathrm{d}}=200 \mathrm{psec}$. The question is now; what are the time delays at $\mathrm{f} 0 \pm(100 \mathrm{MHz})$ ?

Substituting these values in eqn. 3.11.a and solving $\mathrm{f}_{\varphi=0}$ at $\mathrm{f}_{0}=1 \mathrm{GHz}$, we find $\mathrm{f}_{\varphi=0}=-3.28 \mathrm{GHz}$. The maximum time delay variation inside the frequency band is found from eqn. 3.5. In Fig. 3.8, curve (a) shows $\varphi$ vs. $\mathrm{f}$ for the simulation results of the RC delay block. Table 3.1.a shows the delays which results from calculation, simulation and the relative error of calculation results to simulation results. 
Example 2: An LC delay line (Fig. 3.6.b), with $\mathrm{L}=1 \mathrm{nH}, \mathrm{C}=253 \mathrm{fF}$, at $\mathrm{f}_{0}=1 \mathrm{GHz}$ after the 12 th cell shows a delay equal to $\tau_{\mathrm{d}}=204.2 \mathrm{psec}$ (a delay value near to example 1). Again we want to find the time delays at $\mathrm{f}_{0} \pm(100 \mathrm{MHz})$. Eqn. 3.13.b can be used as $2 \pi(\mathrm{LC})^{0.5} \mathrm{f}_{0}=0.1<<1$. We find the value of $\mathrm{f}_{\varphi=0}$ for every LC delay cell which is loaded with $\mathrm{Z}_{0}=(\mathrm{L} / \mathrm{C})^{0.5}$. For every LC cell the calculation result is $\mathrm{f}_{\varphi=0}=-10 \mathrm{MHz}$. Because the loaded LC cells are cascaded then eqn. 3.14 can be used for finding the overall $f_{\varphi=0}$ of the LC delay line which results in: $f_{\varphi=0, \text { Circuit }}=f_{\varphi=0 \text {,every cell }}=-10 \mathrm{MHz}$. In Fig. 3.8 , curve (b) shows $\varphi$ vs. $f$ for the simulation results of the LC delay circuit. Table 3.1.b shows the delays which results from calculation, simulation and the relative error between them.

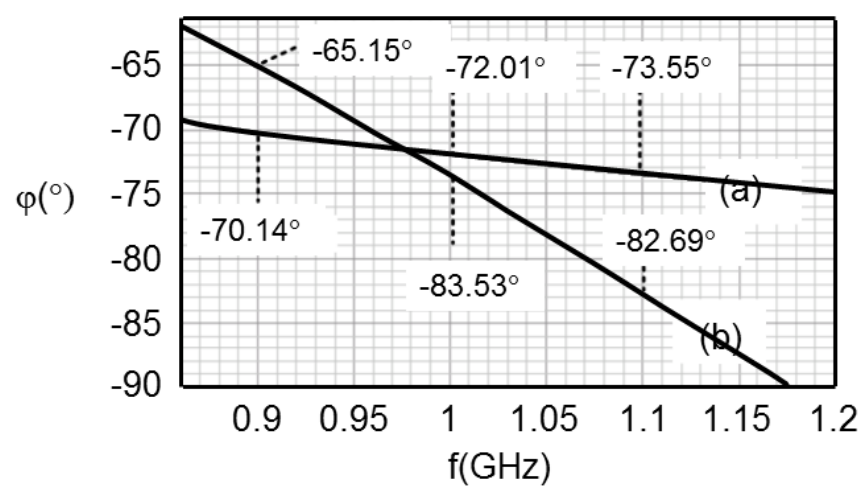

Fig. 3.8. Curves (a) and (b) shows $\varphi$ vs. f transfer function of an RC delay cell and an LC delay circuit respectively 


\begin{tabular}{|c|c|c|c|}
\hline RC delay cell & $0.9 \mathrm{GHz}$ & $1 \mathrm{GHz}$ & $1.1 \mathrm{GHz}$ \\
\hline$\tau_{\mathrm{d}}(\mathrm{ps})$ calculation & 215.4 & 200 & 184.6 \\
\hline$\tau_{\mathrm{d}}(\mathrm{ps})$ simulation & 216.4 & 200 & 185.7 \\
\hline Error $(\%)$ & 0.5 & 0 & 0.6 \\
\hline
\end{tabular}

(a)

\begin{tabular}{|c|c|c|c|}
\hline LC delay circuit & $0.9 \mathrm{GHz}$ & $1 \mathrm{GHz}$ & $1.1 \mathrm{GHz}$ \\
\hline$\tau_{\mathrm{d}}(\mathrm{ps})$ calculation & 204.4 & 204.2 & 204 \\
\hline$\tau_{\mathrm{d}}(\mathrm{ps})$ simulation & 201.1 & 204.2 & 208.8 \\
\hline Error (\%) & -1.6 & 0 & 2.3 \\
\hline
\end{tabular}

(b)

Table 3.1. comparison of the calculation and simulation results for (a)RC delay cell (b) LC delay circuit

The results show that the formula predicts the simulation with quite acceptable accuracy (a few $\%$ over $+/-10 \%$ frequency variation around the center frequency).

\subsection{Conclusion}

This chapter shows that group delay alone is not sufficient for characterizing the delay variation in delay blocks and proposes $\mathrm{f}_{\varphi=0}$ as an additional criterion. The combination of $\mathrm{f}_{\varphi=0}$ with the group delay allows for estimating the delay variations over a certain frequency band around center frequency $\mathrm{f}_{0}$. Equations for $\mathrm{f}_{\varphi=0}$ for an RC delay cell and LC delay section have been derived and compared to simulations, showing the prediction is within few percent over $10 \%$ frequency variation. 


\section{References}

[1] Robert J. Mailloux., "Phased Array Antenna Handbook, SecondEdition" (Artech House Antennas and Propagation Library,2005)

[2] Kendall L.Su., “Analog filters" (Chapman \& Hall press,1996)

[3] K. D. D., "Are group delay time and/or phase delay time useful parameters for defining low distortion transmission." Communication, IEEE Transaction on. , vol. COM-21. pp. 1446- 1448, Dec. 1973

[4] Mueller, M., "Signal Delay" Communications, IEEE Transactionon Volume 23, Issue 11, Page(s):1375 - 1378, Nov 1975

[5] Frank Ellinger.,"Radio Frequency Intergrated Cicruits and Technologies" (Springer 2007) 


\section{Chapter 4}

\section{Phased-Array Antenna Beam Squinting Related to Frequency Dependency of Delay Circuits ${ }^{1}$}

\subsection{Introduction}

Phased-array antenna systems have wide range of applications for example in radar, imaging and communication systems [1], [2], [3]. The design of phased array systems is challenging, especially when a wide band of operation is required. An important phenomenon that can limit bandwidth in phased array antenna systems is beam squinting [1], i.e. the changing of the beam direction as a function of the operating frequency, see Fig. 4.1.

\footnotetext{
${ }^{1}$ This chapter is an exact copy of the following paper:

S. K. Garakoui, E. A. M. Klumperink, B. Nauta and F. E. van Vliet, "Phased-array antenna beam squinting related to frequency dependency of delay circuits," EuRad Conf., 2011, pp. 416-419.
} 


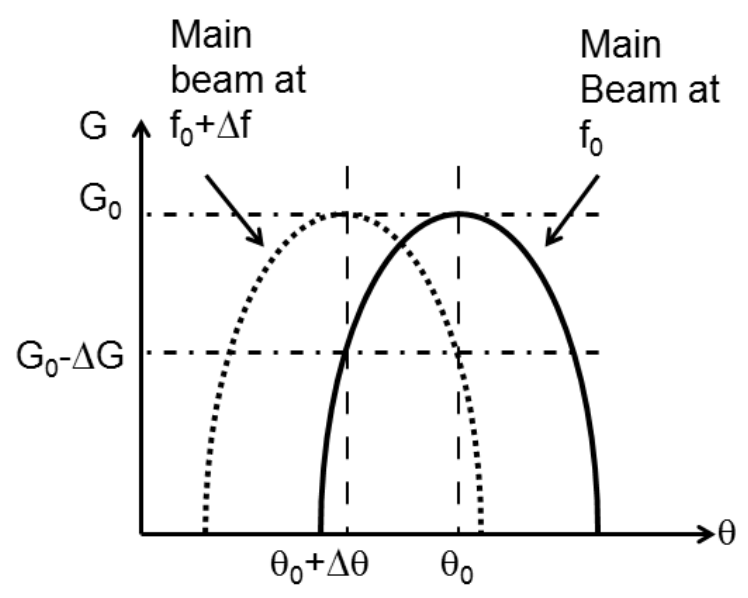

Fig. 4.1. Antenna pattern illustrated beam squint

Beam squinting, in words, means that an antenna pattern points to $\theta_{0}+\Delta \theta$ at frequency $f_{0}+\Delta f$ instead of $\theta_{0}$, which was the pointing direction at frequency $\mathrm{f}_{0}$. With Fig. 4.1 , we see that this might also be interpreted as a reduction of the gain in the direction $\theta_{0}$, limiting the usable bandwidth of the system. The goal of this chapter is to quantify beam squinting, i.e. express $\Delta \theta$ as a function of $\Delta \mathrm{f}$. To clarify the approach in our work compared to previous work, Fig. 4.2 shows the phase frequency characteristics of an ideal phase-shifter, an ideal time-delay and a practical time-delay circuit used in a frequency band centered on $\mathrm{f}_{0}$. In [1] a beam squinting formula has been derived for phased array systems realized with ideal phase-shifters. Also, in [4] a beam squinting formula has been derived for phased-array systems with ideal time-delays and phase-shifters used at different hierarchical levels. Here we will derive a beam squinting formula based on the tangent line in Fig. 4.2, which models practical time delay non-ideality using the criterion $\mathrm{f}_{\varphi=0}[6]$. We extract $\mathrm{f}_{\varphi=0}$ from the phase transfer function of a practical (non-ideal) delay cell to quantify variations of the delay with frequency centered on $\mathrm{f}_{0}$. 


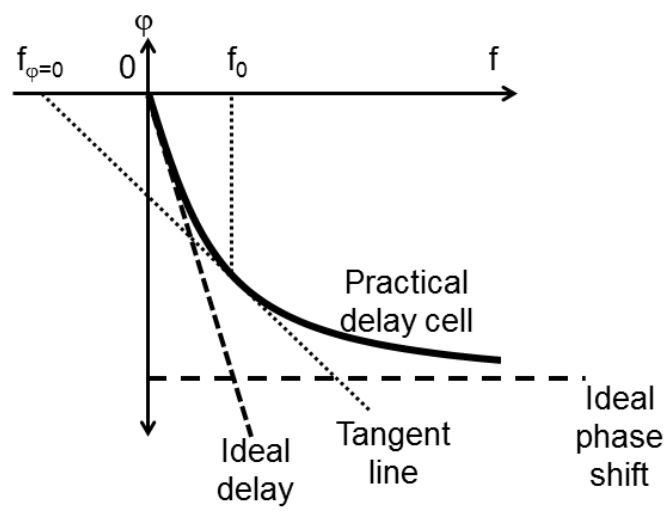

Fig. 4.2. Phase-frequency characteristic of a practical delay circuit, its tangent approximation line in comparison to an ideal delay, and an ideal phase-shifter circuit

Criterion $\mathrm{f}_{\varphi=0}$ can be used for arbitrary delay cells, for example it fits well to $g_{m}-(R) C$ and LC delay cells. The main reason for its use, however, is that it is proven to be invariant for cascaded cells[6] which allows establishing a direct relationship between phased array system specifications and the delay cell requirements.

The relation between $\Delta \theta$ and $\Delta \mathrm{f}$ is established in section 4.2. With the help of a two-term Taylor approximation, formulated in section 4.3 , we develop the beam squinting relation in the presence of timedelay nonlinearities in section 4.4. Finally the relation between system specifications and delay-element requirements is illustrated in section 4.5 after which it is applied to a typical example in section 4.6.

\subsection{Relation between $\Delta \theta$ and $\Delta f$}

Fig. 4.3 shows a typical linear phased array where $\mathrm{d}$ is the distance between any two adjacent antenna elements and $-\pi / 2<\theta<\pi / 2$ is the spatial direction of the beam with respect to bore sight. $\mathrm{D}_{0}, . ., \mathrm{D}_{\mathrm{N}-1}$ are the delay blocks after the antenna elements. 


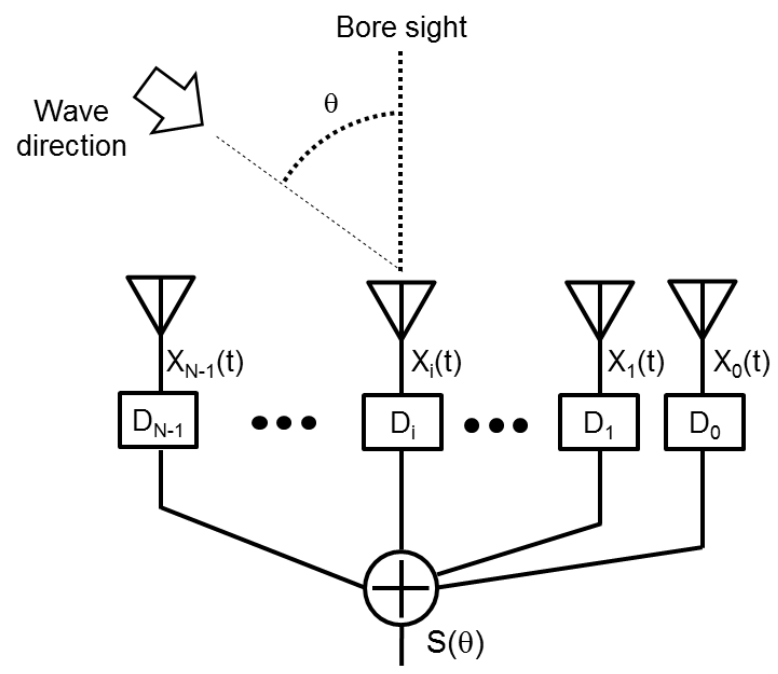

Fig. 4.3. Linear phased array antenna system with equally spaced antennas

Eqn. 4.1 defines the beam pattern $S(\theta)[7]$ :

$S(\theta)=\frac{S_{e}(\theta)}{\sum_{i=0}^{N-1} \alpha_{i}} \sum_{i=0}^{N-1} \alpha_{i} e^{j 2 \pi f\left(i \frac{d}{c} \sin \theta-i \frac{d}{c} \sin \theta_{0}\right)}$

$\mathrm{S}_{\mathrm{e}}(\theta)$ is the antenna element pattern, $\alpha_{\mathrm{i}}$ is the "amplitude tapering factor" of the $\mathrm{i}^{\text {th }}$ antenna route, $\mathrm{c}$ is the speed of light and $\theta_{0}$ the direction of the main beam. Eqn. 4.2 shows the values of delay in delay blocks to have beam direction toward $\theta_{0}$ :

$t_{D i}=i \cdot \frac{d}{c} \cdot \sin \theta_{0} ; i=0, \ldots, N-1$

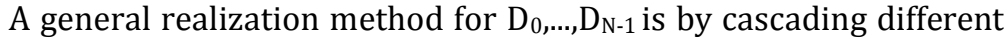
numbers of identical delay cells (Fig. 4.4). Delay cells for instance are realized by $\mathrm{g}_{\mathrm{m}}$-RC or LC circuits. 


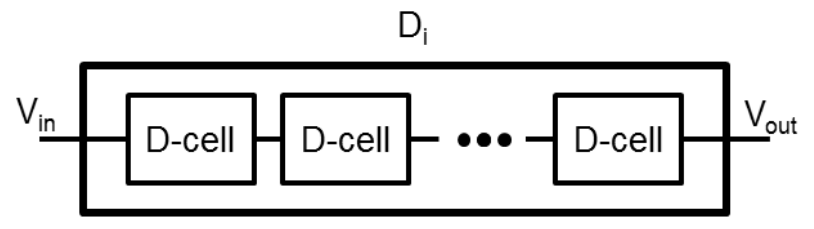

Fig. 4.4. Delay D synthesized from cascaded delay cells.

Eqn. 4.2 reveals that the delay of each delay block $\left(\mathrm{D}_{0}, \ldots, \mathrm{D}_{\mathrm{N}-1}\right)$ is an integer multiple of $t_{D 1}=(d / c) \operatorname{Sin}\left(\theta_{0}\right)$. For practical implementations, $t_{D 1}=t_{D 1}(f)$. By substituting $t_{D 1}(f)=(d / c) \operatorname{Sin}\left(\theta_{0}\right)$ in eqn. 4.1 we get:

$S(\theta)=\frac{S_{e}(\theta)}{\sum_{i=0}^{N-1} \alpha_{i}} \sum_{i=0}^{N-1} \alpha_{i} e^{j 2 \pi f i\left(\frac{d}{c} \sin \theta-t_{D 1}(f)\right)}$

The beam direction at frequency $\mathrm{f}$ is the value of $\theta$ that results in a maximum value of $S(\theta)$, which happens when all antenna contributions align up in phase, i.e. [7]:

$\frac{d}{c} \sin \theta-t_{D 1}(f)=0$

Suppose that at $\mathrm{f}_{0}$ the beam direction is toward $\theta_{0}$, then:

$\frac{d}{c} \sin \theta_{0}-t_{D 1}\left(f_{0}\right)=0$

If the operating frequency varies from $\mathrm{f}_{0}$ to $\mathrm{f}_{0}+\Delta \mathrm{f}$, then due to beam squinting the beam points towards direction $\theta_{0}+\Delta \theta$. Substituting $\mathrm{f}_{0}+\Delta \mathrm{f}$ and $\theta 0+\Delta \theta$ in eqn. 4.4 renders:

$\frac{d}{c} \sin \left(\theta_{0}+\Delta \theta\right)-t_{D 1}\left(f_{0}+\Delta f\right)=0$

The beam squinting formula $\Delta \theta=\Delta \theta(\Delta \mathrm{f})$ can be derived by solving eqn. 4.6. However, because terms at the left side of eqn. 4.6 are nonlinear functions of $\theta_{0}+\Delta \theta$ and $\mathrm{f}_{0}+\Delta \mathrm{f}$, its analytical solution can be 
complicated which is inconvenient for design purposes. Therefore, we will approximate both nonlinear terms of eqn. 4.6 by a linear 2-term Taylor series approximation.

\section{3 $f_{\varphi=0}$ : A criterion for delay versus frequency variations}

In order to linearly approximate $t_{D 1}\left(f_{0}+\Delta f\right)$, we use a recently introduced criterion $f_{\varphi=0}$ [6] to quantify delay variations over frequency. The fact that $\mathrm{f}_{\varphi=0}$ is not affected by cascading of identical cells makes it particularly attractive for designing cascaded delay circuits as in Fig. 4.4. Fig. 4.5, shows the $\mathrm{f}_{\varphi=0}$ for the phase transfer function of $t_{D 1}(f)$. At operating frequency of $f_{0}, f_{\varphi=0, D 1}$ is defined as the cross-point of the frequency axis with the tangent line $L$ to the phase characteristic at $\left(\mathrm{f}_{0}, \varphi_{\mathrm{D} 1}\left(\mathrm{f}_{0}\right)\right)$. By inspection of Fig. 4.5 we can write $\mathrm{f}_{\varphi=0, \mathrm{D} 1}$ as [6]:

$$
f_{\varphi=0, D 1}=f_{0}-\frac{\varphi_{D 1}\left(f_{0}\right)}{\left.\frac{\partial \varphi_{D 1}(f)}{\partial f}\right|_{f_{0}}}
$$

The delay of the delay block $D_{1}$ at frequency $f_{0}$ is equal to $t_{D 1}\left(f_{0}\right)=-$ $\varphi_{\mathrm{D} 1}\left(\mathrm{f}_{0}\right) /\left(2 \pi \mathrm{f}_{0}\right)$. For finding the delay at $\mathrm{f}_{0}+\Delta \mathrm{f}$, we linearly approximate the curve with its tangent (L line) (eqn. 4.8) [6].

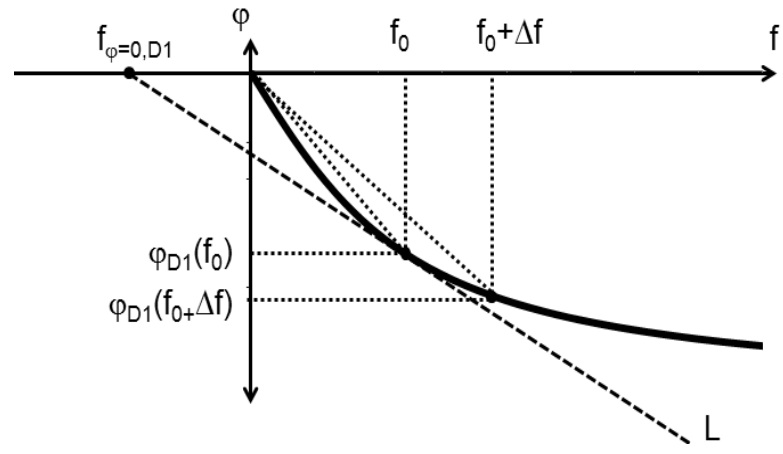

Fig. 4.5. $\mathrm{f}_{\varphi=0}$ for delay $\mathrm{D}_{1}$ that is operating at $\mathrm{f}_{0}$ 


$$
t_{D 1}\left(f_{0}+\Delta f\right) \approx t_{D 1}\left(f_{0}\right)\left(1+\frac{\frac{f_{\varphi=0, D 1}}{f_{0}}}{1-\frac{f_{\varphi=0, D 1}}{f_{0}}} \cdot \frac{\Delta f}{f_{0}}\right)
$$

As it is shown in Fig. 4.4, $\mathrm{D}_{1}$ is synthesized with cascaded identical delay cells and $\mathrm{f}_{\varphi=0, \mathrm{D}-\text { cell }}$ for each delay cell is[6]:

$$
f_{\varphi=0, D-c e l l}=f_{0}-\frac{\varphi_{D-\text { cell }}\left(f_{0}\right)}{\left.\frac{\partial \varphi_{D-c e l l}(f)}{\partial f}\right|_{f_{0}}}
$$

It can be proven that $f_{\varphi=0, D 1}=f_{\varphi=0, D-c e l l}[6]$, which is illustrated in Fig. 4.6. Substituting $\mathrm{f}_{\varphi=0, \mathrm{D} \text {-cell }}$ in eqn. 4 . 8, we find:

$$
t_{D 1}\left(f_{0}+\Delta f\right) \approx t_{D 1}\left(f_{0}\right)\left(1+\frac{\frac{f_{\varphi=0, D-\text { cell }}}{f_{0}}}{1-\frac{f_{\varphi=0, D-\text { cell }}}{f_{0}}} \cdot \frac{\Delta f}{f_{0}}\right)
$$

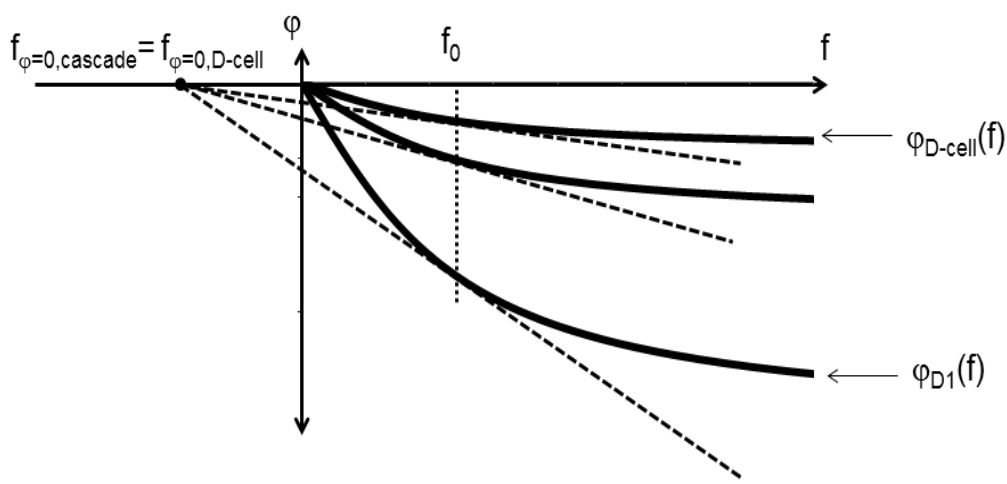

Fig. 4.6. Cascading identical delay cells does not affect $\mathrm{f}_{\varphi=0}$ 
Eqn. 4.10 shows that we can estimate delay of $t_{D 1}\left(f_{0}+\Delta f\right)$ via $t_{D 1}\left(f_{0}\right)$ and also $f_{\varphi=0}$ of its constituent delay cells (D-cells). In the next section this result is used to derive $\Delta \theta(\Delta \mathrm{f})$.

\subsection{Beam squinting formula}

We will now use the linear approximation derived in the previous section to derive the beam squint formula. Substituting eqn. 4.10 in 4.6 and some rewriting gives:

$$
\frac{d}{c}\left(\sin \theta_{0}+\Delta \theta \cdot \cos \theta_{0}\right)-t_{D 1}\left(f_{0}\right)\left(1+\frac{\frac{f_{\varphi=0, D-\text { cell }}}{f_{0}}}{1-\frac{f_{\varphi=0, D-\text { cell }}}{f_{0}}} \cdot \frac{\Delta f}{f_{0}}\right)=0
$$

Rearranging terms of eqn. 4.11 results in:

$$
\begin{aligned}
& \left(\frac{d}{c} \sin \theta_{0}-t_{D 1}\left(f_{0}\right)\right)+\left(\frac{d}{c} \cdot \Delta \theta \cdot \cos \theta_{0}-t_{D 1}\left(f_{0}\right) \frac{\frac{f_{\varphi=0, D-c e l l}}{f_{0}}}{1-\frac{f_{\varphi=0, D-c e l l}}{f_{0}}} \cdot \frac{\Delta f}{f_{0}}\right) \\
& =0
\end{aligned}
$$

The part between the first brackets is zero according to eqn. 4.5. Therefore the remaining part of eqn. 4.12 must be equal to zero too, which allows to easily solving $\Delta \theta$ as a function of $\Delta \mathrm{f}$ :

$\Delta \theta=\frac{1}{f_{0}} \frac{c}{d} \cdot \frac{t_{D 1}\left(f_{0}\right)}{\cos \theta_{0}} \frac{\frac{f_{\varphi=0, D-\text { cell }}}{f_{0}}}{1-\frac{f_{\varphi=0, D-\text { cell }}}{f_{0}}} \cdot \Delta f$ 
This can be further simplified, substituting $\mathrm{t}_{\mathrm{D} 1}\left(\mathrm{f}_{0}\right)$ from eqn. 4.5 in eqn. 4.13 . The result is eqn. 4.14 or the beam squinting formula:

$$
\Delta \theta=\frac{\tan \theta_{0}}{f_{0}} \frac{\frac{f_{\varphi=0, D-\text { cell }}}{f_{0}}}{1-\frac{f_{\varphi=0, D-\text { cell }}}{f_{0}}} \cdot \Delta f
$$

Thus we see that $f_{\varphi=0, D-c e l l} / f_{0}$ is crucial for beam squint estimation. For a phased array realized by ideal time-delay cells, $\mathrm{f}_{\varphi=0, \mathrm{D} \text {-cell }}$ is equal to zero and we find indeed zero squinting $(=0)$. For a phased array realized by ideal phase-shifters, $\mathrm{f}_{\varphi=0, \mathrm{D} \text {-cell }}$ is equal to $-\infty$ rendering the result from [1]:

$$
\Delta \theta=-\frac{\tan \theta_{0}}{f_{0}} \Delta f
$$

In the next section as an example $\mathrm{f}_{\varphi=0, \mathrm{D}-\text { cell }}$ will be derived for an allpass delay cell.

\subsection{Beam squinting with all-pass delay cells}

One possibility is to realize a time-delay cell by implementing a first order all-pass filter. The ideal transfer function of this all-pass filter is given as:

$H_{\text {all-pass }}(f)=\frac{\left(1-j \frac{f}{f_{p}}\right)}{\left(1+j \frac{f}{f_{p}}\right)}$

We use eqn. 4.9 to find a normalized graph of the $f_{\varphi=0, D-c e l l}$ versus $\mathrm{f}_{0} / \mathrm{f}_{\mathrm{p}}$. Normalization gives us a generalized curve to be used for $1^{\text {st }}$ order delay cells with any value of the pole frequency $\left(f_{p}\right)$ and the operating frequency $\left(f_{0}\right)$. The curve helps to find an $f_{p}$ for the delay cell 
to keep the beam squinting below a requested range. The phase transfer function of the $1^{\text {st }}$ order all-pass cell is:

$$
\varphi_{\text {all-pass }}(f)=-2 \operatorname{atan}\left(\frac{f}{f_{p}}\right)
$$

This leads to amount of the delay per all-pass cell at $\mathrm{f}_{0}$ :

$t_{\text {all-pass }}\left(f_{0}\right)=\frac{2 \operatorname{atan}\left(\frac{f}{f_{p}}\right)}{2 \pi f_{0}}$

Substitution of eqn. 4.17 in eqn. 4.9 and normalization for $\mathrm{f}_{0}$ results eqn. 4.19 which its graph is Fig. 4.7.

$$
\frac{f_{\varphi=0, a l l-p a s s}}{f_{0}}=1-\operatorname{atan}\left(\frac{f_{0}}{f_{p}}\right)\left(\frac{f_{0}}{f_{p}}+\frac{f_{p}}{f_{0}}\right)
$$

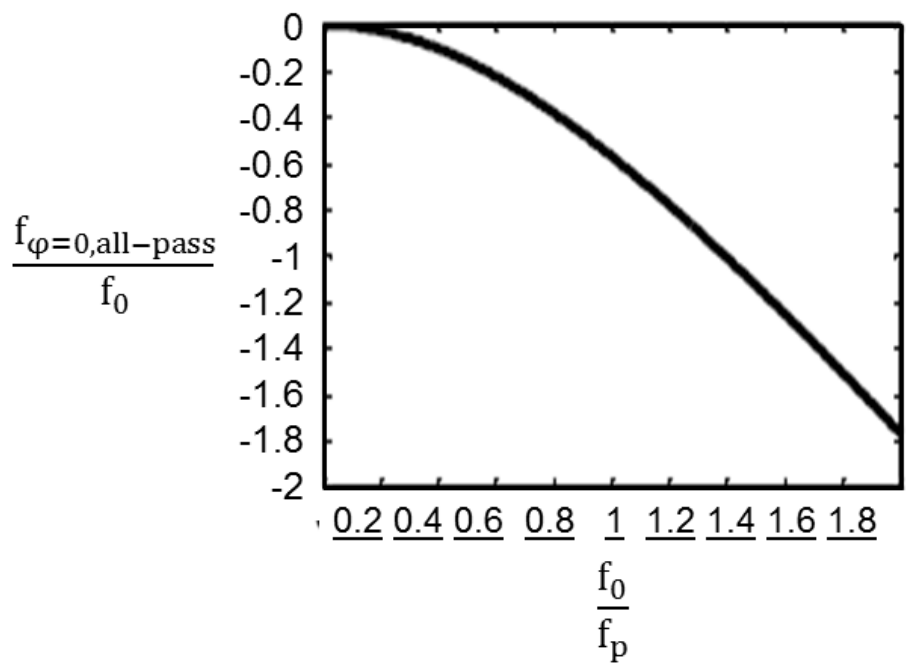

Fig. 4.7. Normalized $f_{\varphi=0}$ versus $f_{0}$ curve for all-pass cells 
This graph is used to find minimum value of $f_{p}$ of a $1^{\text {st }}$ order allpass delay cell that satisfies a certain permitted amount of the beam squinting. Suppose an amount of $\Delta \theta / \Delta \mathrm{f}$ for the phased array antenna is permitted, then via eqn. $4.14, \mathrm{f}_{\varphi=0, \mathrm{D} \text {-cell }} / \mathrm{f}_{0}$ is found and then via the graph of the Fig. 4.7, $\mathrm{f}_{0} / \mathrm{f}_{\mathrm{p}}$ and consequently the minimum value of $\mathrm{f}_{\mathrm{p}}$ can be found.

\subsection{Example}

We show how we can find the minimum required pole frequency $\left(\mathrm{f}_{\mathrm{p}}\right)$ of an all-pass delay cell starting from a beam squinting specification and verify the design by simulations. We aim to keep the beam squinting in a defined range. As an example, we assume a linear phased array antenna system with the following characteristics: $\mathrm{N}=100$ antenna elements, operating frequency $\mathrm{f}_{0}=10 \mathrm{GHz}$, element distance $d=\lambda / 2=1.5 \mathrm{~cm}$ and a maximum steering angle $\theta_{0}=60^{\circ}$. Assume furthermore that an absolute beam squinting per frequency deviation $(\Delta \theta / \Delta \mathrm{f})$ of less than $3^{\circ} / \mathrm{GHz}$ is required:

$\mathrm{f}_{\varphi=0 \text {,all-pass }} / \mathrm{f}_{0}$ is found by substituting $\Delta \theta / \Delta \mathrm{f}, \mathrm{f}_{0}$ and $\theta_{0}$ in beam squinting formula (eqn. 4.14). The result is: $\mathrm{f}_{\varphi=0 \text {,all-pass }} / \mathrm{f}_{0}>-0.43$. Substitution of $\mathrm{f}_{\varphi=0 \text {,all- }}$ pass $/ f_{0}>-0.43$ in graph of Fig. 4.7 results $f_{0} / f_{p}<0.85$. Because $f_{0}=10 \mathrm{GHz}$, then we will find $f_{p}$ of the all-pass delay cell which is $f_{p}>11.8 \mathrm{GHz}$.

Therefore, to obtain a beam squinting less than $3^{\circ} / \mathrm{GHz}$, the allpass delay cell which we use in the phased array must have a pole frequency $\left(\mathrm{f}_{\mathrm{p}}\right)$ larger than $11.8 \mathrm{GHz}$. The delay of the cell is found from eqn. 4.18 which is $t_{\text {all-pass }}=21.4$ ps. The maximum required delay for the phased array (Delay of $\mathrm{D}_{\mathrm{N}-1}$ block of Fig. 4.3 is found by substituting values of $\mathrm{i}=\mathrm{N}-1=99, \mathrm{~d}=\lambda / 2=1.5 \mathrm{~cm}, \mathrm{c}=$ light speed and $\theta_{0}=60^{\circ}$ in eqn. 4.2 . The result is: $t_{D 99}=2143$ psec. Maximum number of cascaded delay cells to synthesize $t_{D 99}$ is found from $t_{D 99} / t_{\text {all-pass }}$ which is: 100.14 . Therefore 101 cascaded all-pass delay cells are required for synthesizing $\mathrm{D}_{99}$ delay block in the phased array antenna system.

Finally, to verify the precision of our method, we simulate the phased array with delay blocks as synthesized above, to check if the beam squinting is in the requested range. Table 4.1 compares the 
simulated and required beam squinting for different frequency offsets from $\mathrm{f}_{0}=10 \mathrm{GHz}$. The error is calculated via: Error $=\left(\Delta \theta_{\text {Simulated }^{-}}\right.$ $\left.\Delta \theta_{\text {Required }}\right)$.It shows that up to $15 \mathrm{GHz}\left(50 \%\right.$ offset from $\left.\mathrm{f}_{0}\right)$, the absolute amount of the error remains less than $14 \%$ of $\Delta \theta_{\text {Required. This }}$ shows that via our method we can design delay cells to keep the beam squinting in the requested range.

\begin{tabular}{|c|c|c|c|c|c|}
\hline $\mathrm{f}_{0}=10 \mathrm{GHz}$ & $\begin{array}{c}\Delta \mathrm{f}=0.1 \\
\mathrm{GHz}\end{array}$ & $\begin{array}{c}\Delta \mathrm{f}=0.5 \\
\mathrm{GHz}\end{array}$ & $\begin{array}{c}\Delta \mathrm{f}=1 \\
\mathrm{GHz}\end{array}$ & $\begin{array}{c}\Delta \mathrm{f}=2 \\
\mathrm{GHz}\end{array}$ & $\begin{array}{c}\Delta \mathrm{f}=5 \\
\mathrm{GHz}\end{array}$ \\
\hline $\begin{array}{c}\Delta \theta^{\circ} \\
\text { Simulated }\end{array}$ & 0.34 & 1.67 & 3.23 & 6.14 & 13.4 \\
\hline $\begin{array}{c}\Delta \theta^{\circ} \\
\text { Required }\end{array}$ & 0.3 & 1.5 & 3 & 6 & 15 \\
\hline Error $^{\circ}$ & 0.04 & 0.17 & 0.23 & 0.14 & -1.6 \\
\hline
\end{tabular}

Table 4.1. Comparison between the simulated and the required beam squinting

\subsection{Conclusion}

A general formula was derived to estimate beam squinting in phased array antenna systems. This formula is particularly useful to estimate beam squinting of wide band (time-delay based) phased array antenna systems. To estimate beam squinting we first calculate the criterion $\left(f_{\varphi=0, D-c e l l}\right)$ from the phase transfer function of the delay cell. Then find the beam squinting via the beam squinting formula with $\mathrm{f}_{\varphi=0, \mathrm{D} \text {-cell }}$ as a parameter. Also the beam squinting formula can be used to estimate the amount of $f_{\varphi=0, D-c e l l}$ to keep the beam squinting in a permitted range. We designed a phased array with $1^{\text {st }}$ order all-pass delay cells via this method. The method is suitable for non-ideal timedelay elements as well as for non-ideal phase-shifters or any other element where the phase transfer can be approximated linearly. 


\section{References}

[1] R.J.Mailloux,"Phased array antenna handbook", Artech house, 2007.

[2] Nicholas Fourikis, "Advanced Array Systems, Applications and RF technology", Academic Press, 2000.

[3] Paramesh, J," A four-antenna receiver in 90-nm CMOS for beamforming and spatial diversity ", IEEE Journal of Solid-State Circuits (JSSC), Vol: 40, no: 12, pp. 2515 - 2524, Dec. 2005.

[4] Yajima, M, "Beam Pointing Error of Wideband Planar Phased Array", IEEE International Conference on Communications, pp: 41614166, 2006.

[5] Ta-Shun Chu,"An Integrated Ultra-Wideband Timed Array Receiver in $0.13 \mu \mathrm{m}$ CMOS Using a Path-Sharing True Time Delay Architecture“, Journal of Solid-State Circuits (JSSC), Vol: 42, no: 12, pp. 2834-2850, 2007.

[6] Garakoui, S., K., "Time delay circuits: A quality criterion for delay variations versus frequency," International Symposium on Circuits and Systems, pp.4281-4284, 2010.

[7] Hubegt Visser,"Array and Phased Array Antenna Basics", Wiley 2005.

[8] Frank Ellinger,"Radio Frequency integrated circuits and technologies", Springer 2007 


\section{Chapter 5}

\section{Frequency Limitations of First-Order $\mathrm{g}_{\mathrm{m}}-\mathrm{RC}$ All-pass Delay Circuits ${ }^{1}$}

\subsection{Introduction}

Analog time delay circuits have several applications, for instance compensating delay differences between signal paths, broadband beamforming [1] and equalizing the communication channel for wireline communication [2]. Ideally such delay circuits should have both a constant unity gain and a well-defined constant delay, which does not vary with frequency. However, practical delay circuits do show frequency dependent gain and delay variations. This frequency dependence affects the functionality of systems which exploit delay circuits, limiting their accuracy. For example in time-delay based phased-array antenna systems, a frequency dependent time delay causes a frequency dependency in the beam direction ("beam squint") $[3,4]$.

CMOS is often the desired technology for the implementation of mixed-signal systems. At radio frequencies, OPAMPs are impractical,

1 This chapter is an exact copy of the following paper:

S. K. Garakoui, E. A. M. Klumperink, B. Nauta and F. E. van Vliet, "Frequency Limitations of First-Order gm - RC All-Pass Delay Circuits," IEEE T. on Circuits and Systems II, vol. 60, no. 9, pp. 572-576, Aug. 2013. 
and time delays are typically implemented either by transmission lines [5], LC delay lines [1] or all-pass $g_{m}-R C$ delay circuits $[2,6,7]$. In this paper we focus on circuits which can be implemented in standard CMOS IC-technology at low area cost and low supply voltage. Transmission lines in CMOS require very long (lossy) metal lines to produce a significant amount of delay, while LC delay lines need onchip inductors. The $g_{m}-R C$ all-pass delay circuits proposed in $[2,6,7]$ can produce a given amount of delay much more compactly than inductor based delay cells. Although many trade-offs exist, for instance in achievable frequency, noise, linearity and power consumption, $\mathrm{g}_{\mathrm{m}}-\mathrm{RC}$ all-pass circuits are clearly area and hence cost effective and will be the focus of this chapter.

An ideal $1^{\text {st }}$ order all-pass filter has a pole and zero, and can be written as:

$H(s)=\frac{1-\frac{s}{2 \pi f_{P}}}{1+\frac{s}{2 \pi f_{P}}}$

where $f_{P}$ refers to the pole-frequency. Note that the pole and zeros are positioned at $+/-f_{P}$, resulting in twice the phase and delay of a single-pole system. Also, the gain is 1 and frequency-independent. Fig. 5.1 shows the phase and gain of eqn. 5.1, in comparison to an ideal time-delay cell. The time delay at an operating frequency $\mathrm{f}_{0}$ is equal to $\tau=-\varphi\left(f_{0}\right) /\left(2 \pi f_{0}\right)$. As Fig. 5.1 shows, the delay of a first order all-pass cell is frequency dependent and varies with $\mathrm{f}_{0}$. 

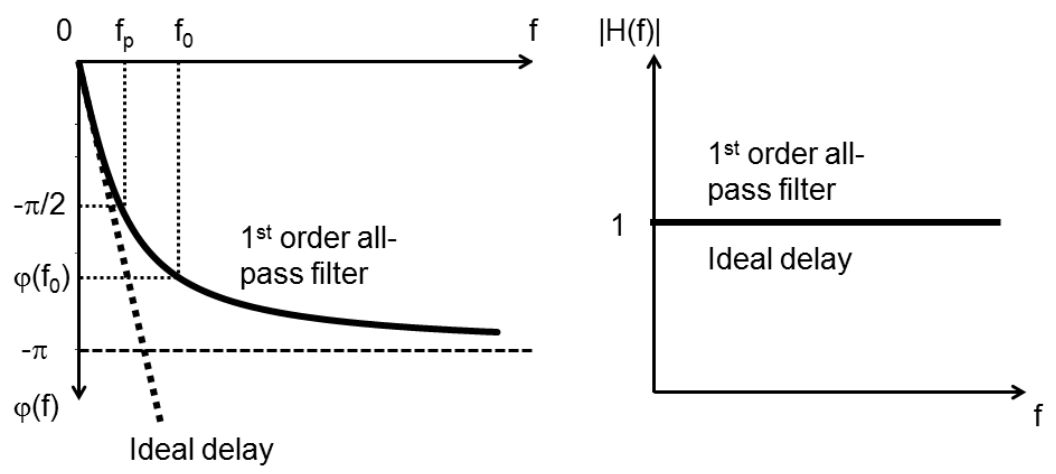

Fig. 5.1. Phase and gain of an ideal versus $1^{\text {st }}$ order delay cell

In general, delay variations [8] and gain variations limit the useful frequency range. What is acceptable depends on system requirements (see for instance [3,4]), and we will assume a maximum allowed gain and delay variation budget. This chapter provides a method to analyze the achievable frequency range of delay circuits given such a budget.

In literature we found several delay circuits, but no comparison of their relative merits, nor a design method to maximize the useful frequency range. This chapter aims at filling this gap.

As low level circuit details critically affect delay cell performance, we will analyze and compare two well-known voltage-mode all-pass circuits. One is the "classical" all-pass delay circuit described in [6], but with much older roots at least dating back to [9]. We will compare this to the "Buckwalter" cell structure proposed in [2]. The circuit of [7] is not considered further, as it is a current-mode circuit complicating comparison, and as it uses 3 stacked transistors which is less suitable for low supply voltages.

We will propose an analysis method which also holds for the practical case where a delay cell operates in a cascade of similar delay cells. The input impedance of the next cell will then load the previous one, while an extra capacitive load $\left(\mathrm{C}_{\mathrm{L}}\right)$ may also be present. The analysis partly builds on [8], where the criterion $\mathrm{f}_{\varphi=0}$ is introduced to 
Chapter 5. Frequency Limitations of First-Order gm-RC All-pass Delay Circuits

quantify variations of delay versus frequency. This figure of merit has some properties [8] that we will exploit, which are briefly summarized below.

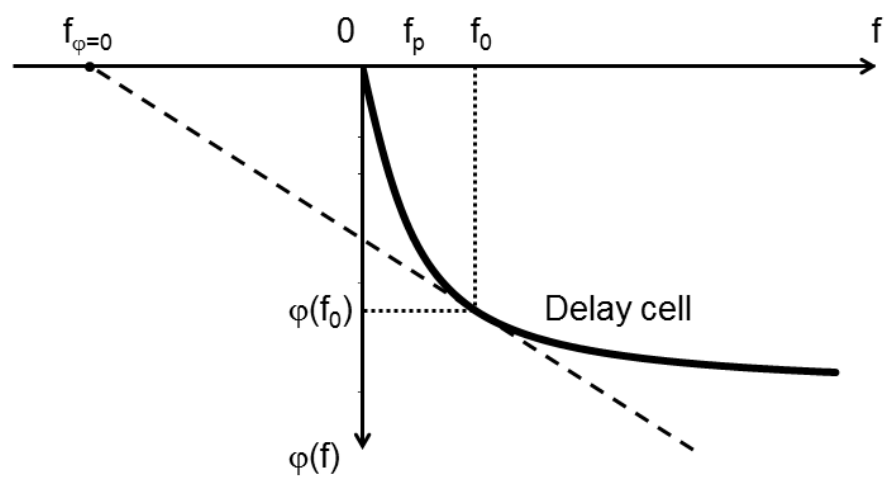

Fig. 5.2. $f_{\varphi=0}$ for a delay cell with operating frequency $f_{0}$

As shown in Fig. 5.2, $\mathrm{f}_{\varphi=0}$ is the frequency where the tangent to the phase transfer function at $f_{0}$ crosses the frequency axis $(\varphi=0)$. For an ideal time delay cell, $f_{\varphi=0} / f_{0}=0$ and for an ideal phase shifter $f_{\varphi=0} / f_{0}=-\infty$ [8]. For a practical delay cell with non-linear phase transfer function a low value of $f_{\varphi=0} / f_{0}$ is desirable. In general, $f_{\varphi=0} / f_{0}$ values can be found from the phase transfer function as [8]:

$\frac{f_{\varphi=0, \text { cell }}}{f_{0}}=1-\frac{\frac{\varphi_{\text {cell }}\left(f_{0}\right)}{f_{0}}}{\left.\frac{\partial \varphi_{\text {cell }}(f)}{\partial f}\right|_{f_{0}}}$

Now, the relative delay variation $\Delta t_{D} / t_{D}\left(f_{0}\right)$ for a frequency variation $\Delta \mathrm{f}$ around $\mathrm{f}_{0}$ is given by [8]: 
$\frac{\Delta t_{D}\left(f_{0}\right)}{t_{D}\left(f_{0}\right)} \approx \frac{\frac{f_{\varphi=0, \text { cell }}}{f_{0}}}{1-\frac{f_{\varphi=0, \text { cell }}}{f_{0}}} \frac{\Delta f}{f_{0}}$

Clearly, if $\mathrm{f}_{\varphi=0} / \mathrm{f}_{0}<<1$, then $\Delta \mathrm{t}_{\mathrm{D}} / \mathrm{t}_{\mathrm{D}}\left(\mathrm{f}_{0}\right) \approx 0$ which means the circuit approximates an ideal delay over frequency band $\Delta \mathrm{f}$.

If we apply eqn. 5.2 to the phase transfer function of the ideal $1^{\text {st }}$ order all-pass cell (eqn.5.1) we find:

$$
\frac{f_{\varphi=0, \text { cell }}}{f_{0}}=1-\frac{\operatorname{atan}\left(f_{0} / f_{P}\right)}{f_{0} / f_{P}}\left(1+\left(f_{0} / f_{P}\right)^{2}\right)
$$

Fig. 5.3 plots numerical values for eqn. 5.4 versus operating frequency $f_{0}$, normalized to pole frequency $f_{p}$. In addition, $f_{p} \tau$ is shown, which slightly varies around 0.25 for $f_{0}=f_{p}$ (phase shift $-\pi / 2$ ). For low relative delay variation at given $\Delta \mathrm{f}$ and $\mathrm{f}_{0}$, Eqn. 5.3 asks for low $\mathrm{f}_{\varphi=0} / \mathrm{f}_{0}$, i.e., large $f_{p}$. However, as $f_{p} \tau$ is around 0.25 , less delay per cell results (roughly $\propto 1 / f_{p}$ ). Fortunately, cascading cells allows for more delay at constant relative delay variation. If cells are identical, $f_{\varphi=0}$ of the cascade is equal to that of a cell [8]. Hence, analyzing $f_{p}$ of a single (loaded) cell is sufficient to characterize a cascade of delay cells with respect to delay variation. 


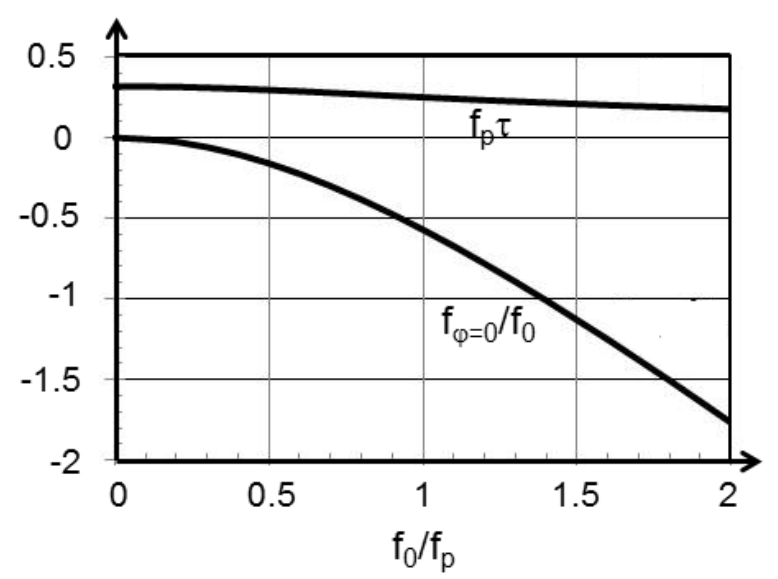

Fig. 5.3. $f_{\varphi=0} / f_{0}$ and $f_{p} \tau$ versus normalized frequency $f_{0} / f_{p}$.

In this chapter, we will show how the maximum value of $f_{p}$ is limited by the circuit topology and technology parameters, where also gain variations induced by parasitic poles will be considered. Note that previous work [8] only modeled ideal low-pass RC and LC delay cell behavior, no all-pass cells. Moreover, no gain variation effects nor parasitic loading effects were modeled, which is way too optimistic. The maximum achievable $\mathrm{f}_{\mathrm{p}}$ will now be analyzed for the "classical" delay circuit in section 5.2 and the "Buchwalter" delay circuit in Section 5.3. Section 5.4 will verify analysis by simulation and compare the relative merits of the circuits. Section 5.5 will present the conclusions.

\subsection{Analysis: The classical delay circuit}

Fig. 5.4 shows the classical all-pass delay cell [6], [9]. Instead of resistors, diode-connected MOSFETs or triode MOSFETs may also be used; however, resistors are preferred for linearity reasons, for having less parasitic capacitance than MOSFETs, and for requiring low voltage headroom. Eqn. 5.5 shows an approximate transfer function. 


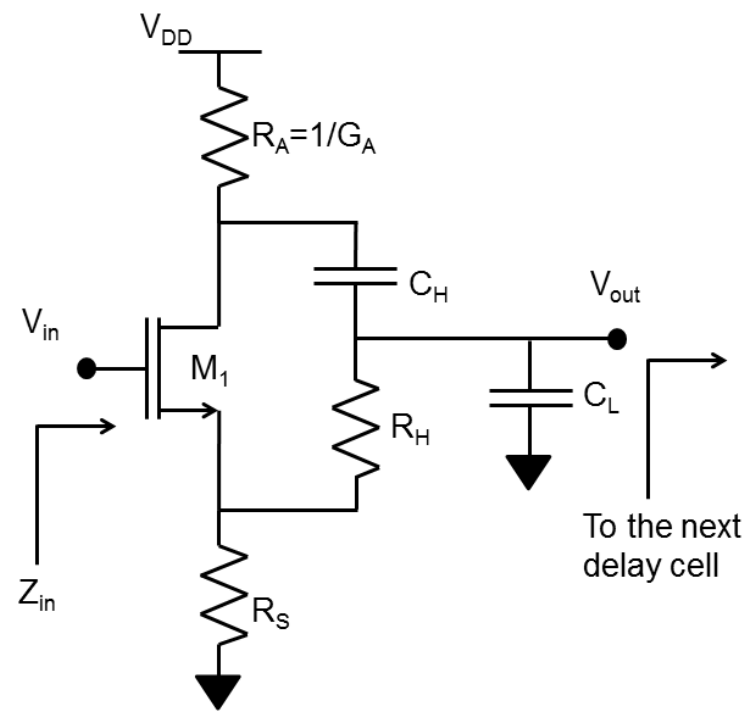

Fig. 5.4. "Classical” first-order all-pass delay cell.

$\frac{V_{\text {out }}}{V_{\text {in }}} \approx \frac{g_{m}}{g_{m}+G_{A}} \cdot \frac{1-R_{H} C_{H} s}{1+\left(R_{H}+\frac{2}{g_{m}+G_{A}}\right)\left(C_{H}+C_{\text {in }}+C_{L}\right) s}$

$\mathrm{C}_{\text {in }}$ is the input capacitance of the next stage. If each delay cell is cascaded with identical delay cells, then $\mathrm{C}_{\mathrm{in}} \approx 2 \mathrm{C}_{\mathrm{gd}}$ (due to the Miller effect on $C_{g d}$ ). Eqn. 5.5 differs from transfer function of eqn. 5.1 because its dc gain is less than one and the absolute value of the pole and zero differ from each other, causing attenuation at high frequencies (see Fig. 5.5). Still, eqn. 5.5 can approximately imitate transfer function of eqn. 5.1; hence, we can reuse eqn. 5.4 provided that two conditions are satisfied as follows:

$$
\begin{aligned}
& \text { 1) } \frac{g_{m}}{g_{m}+G_{A}} \approx 1 \text {, and } \\
& \text { 2) }\left(R_{H}+\frac{2}{g_{m}+G_{A}}\right)\left(C_{H}+C_{i n}+C_{L}\right) \approx R_{H} C_{H}
\end{aligned}
$$


Chapter 5. Frequency Limitations of First-Order gm-RC All-pass Delay Circuits

The second condition ensures proximity of the absolute value of the pole and the zero frequency, which keeps the amount of frequency-dependent gain attenuation small.

We will now assume that the design requirements are

1) dc gain $A_{v 0},\left(0<A_{v 0}<1\right)$;

2) high-frequency attenuation $\leq \Delta \mathrm{Hp}$.

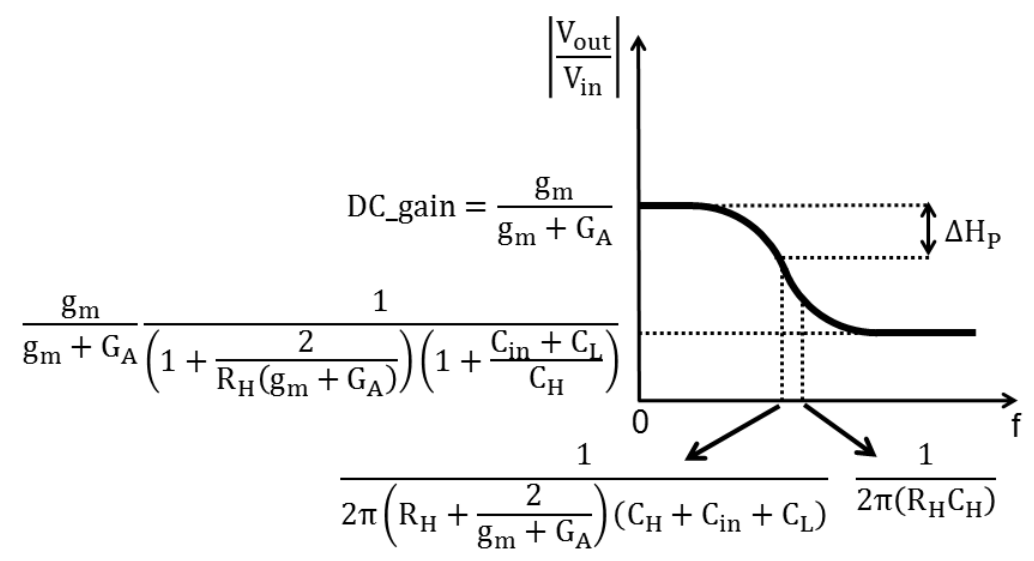

Fig. 5.5. Voltage gain of the "Classical" delay cell

By substituting these values in eqn. 5.5 we get:

$$
\begin{aligned}
& R_{A} \geq \frac{A_{V 0}}{1-A_{V 0}} \frac{1}{g_{m}} \\
& R_{H} \geq \frac{2 A_{V 0}}{g_{m}\left(\frac{1}{\sqrt[4]{2\left(1-\Delta H_{P}\right)^{2}-1}}-1\right)}
\end{aligned}
$$


Chapter 5. Frequency Limitations of First-Order gm-RC All-pass Delay Circuits

$C_{H} \geq \frac{C_{L}+2 C_{g d}}{\left(\frac{1}{\sqrt[4]{2\left(1-\Delta H_{P}\right)^{2}-1}}-1\right)}$

Based on eqn. 5.7 and eqn. 5.8, the condition on the pole and zero frequencies for the "Classical" delay circuit becomes:

$\left|f_{p, C}\right| \leq \frac{1}{2 \pi R_{H} C_{H}} \cdot \sqrt[2]{2\left(1-\Delta H_{P}\right)^{2}-1}$

$\left|f_{z, C}\right| \leq \frac{1}{2 \pi R_{H} C_{H}}$

Note that eqn. 5.9a gives the maximum possible pole frequency, i.e. the lowest delay variation (see eqn. 5.4 and Fig. 5.3).

\subsection{Analysis: The buckwalter delay circuit}

Fig. 5.6 shows the all-pass circuit proposed by Buckwalter [2] implemented using MOSFETs and resistors.

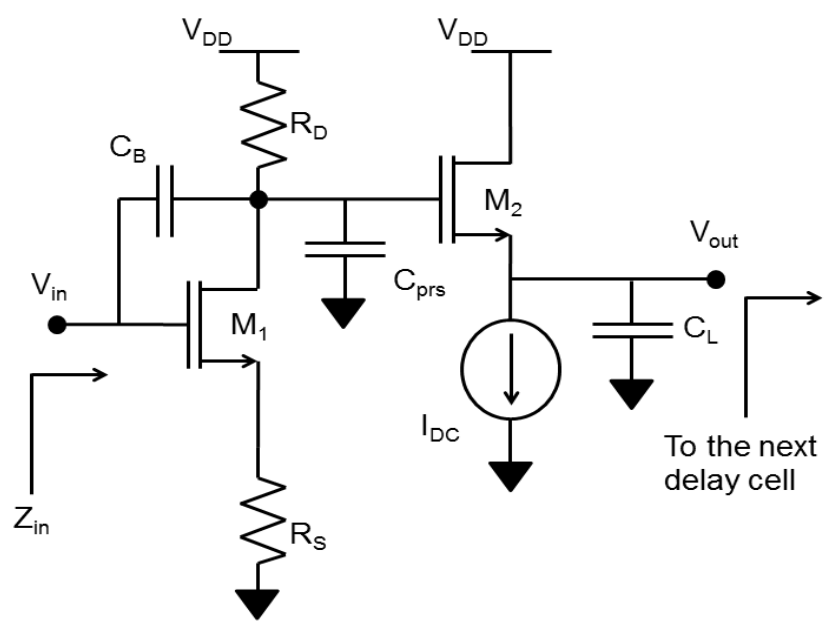

Fig. 5.6. Buckwalter circuit.

93 
As aforementioned, the circuit is cascaded with identical circuits; therefore, the circuit is loaded with impedance that is equal to its input impedance and an extra load capacitance $\mathrm{C}_{\mathrm{L}}$. The input impedance can be simply approximated by evaluating the Miller effects on capacitance values $\mathrm{C}_{\mathrm{B}}$ and $\mathrm{C}_{\mathrm{gs} 1}: \mathrm{C}_{\mathrm{in}} \approx 2 \mathrm{C}_{\mathrm{B}}+\mathrm{C}_{\mathrm{gs} 1} /\left(1+\mathrm{g}_{\mathrm{m}} \mathrm{R}_{\mathrm{S}}\right) \approx$ $2 \mathrm{C}_{\mathrm{B}}$. During the rest of the calculations, the value of $\mathrm{C}_{\mathrm{gd} 1}$ is absorbed inside $C_{B} \cdot C_{p r s}=C_{d b 1}+C_{g d 2}$. The approximate transfer function of the circuit is

$$
\begin{aligned}
& \frac{V_{\text {out }}}{V_{i}} \approx-\frac{R_{D}}{\frac{1}{g_{m 1}}+R_{S}} . \\
& \cdot \frac{1-\frac{1+g_{m 1} R_{S}}{g_{m 1}} C_{B} S}{1+R_{D} C_{B}\left(1+\frac{2}{R_{D} g_{m 2}}\right)\left(1+\frac{\frac{C_{p r s}}{C_{B}}+\frac{C_{L}}{C_{B}} \frac{1}{R_{D} g_{m 2}}}{1+\frac{2}{R_{D} g_{m 2}}}\right) s}
\end{aligned}
$$

The right side of eqn. 5.10 contains two parts. The first part is the dc gain, and the second part is approximately the all-pass transfer function. The initial phase shift at $\mathrm{dc}$ is $\pi$, which we do not consider in our calculations because in processing differential signals, the $\pi$ phase shift can be compensated with interchanging the output signals [2]. To design the delay circuit, we again aim at approximating transfer function (eqn. 5.1). For the unity dc gain, the following condition must be satisfied: $R_{D}=R_{S}+1 / g_{m}$. Fig. 5.7 shows the gain transfer function of the Buckwalter delay circuit with unity dc gain. 


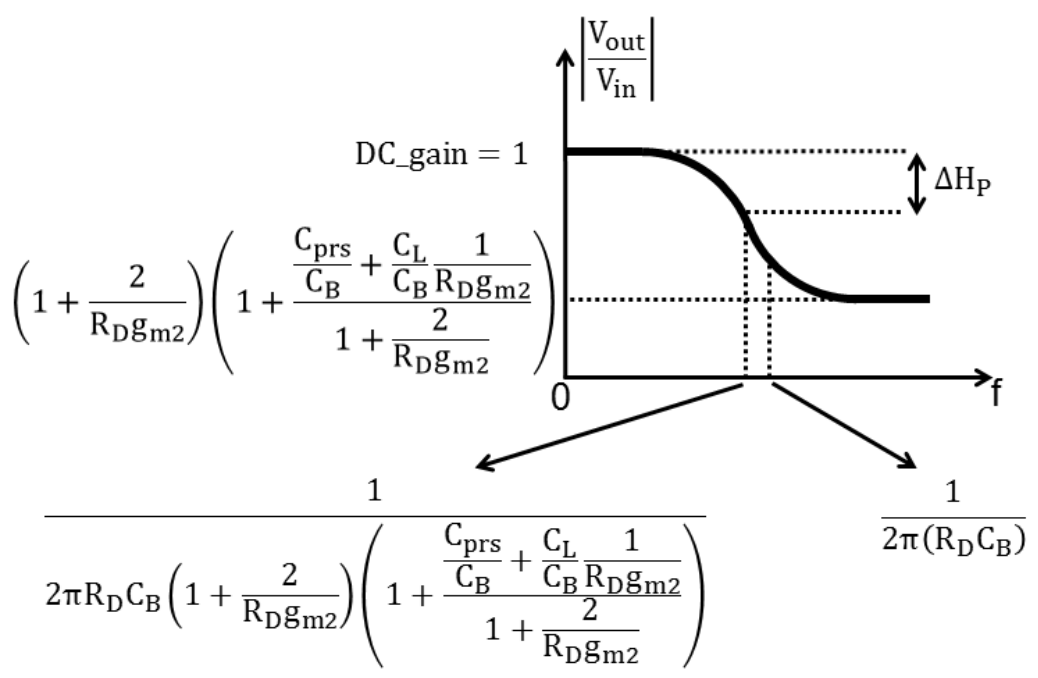

Fig. 5.7. Voltage gain of the Buckwalter delay cell.

Eqn. 5.10 can approximate the transfer function of an all pass delay cell, provided that two conditions are satisfied, i.e.,

1) $\frac{\frac{C_{p r s}}{C_{B}}+\frac{C_{L}}{C_{B} R_{D} g_{m 2}}}{1+\frac{2}{R_{D} g_{m 2}}} \ll 1$, and

2) $\frac{2}{1+R_{D} g_{m 2}} \ll 1$

These conditions cause the absolute values of pole and zero to be close to each other. Suppose there are two design requirements: 1) dc gain $=1$ and 2) at $\mathrm{f}_{0}=\mathrm{f}_{\mathrm{p}}$, the high-frequency attenuation $\leq \Delta \mathrm{H}_{\mathrm{p}}$. Via these assumptions, we can find $g_{m 2}, C_{B}$, and $f_{p}$. Thus

$g_{m 2} \geq \frac{2\left(\sqrt[4]{2\left(1-\Delta H_{P}\right)^{2}-1}\right)}{\left(\frac{1}{g_{m 1}}+R_{S}\right)\left(1-\sqrt[4]{2\left(1-\Delta H_{P}\right)^{2}-1}\right)}$ 


$$
C_{B} \geq \frac{\left(C_{p r s}+\frac{C_{L}}{R_{D} g_{m 2}}\right)}{1+\frac{2}{R_{D} g_{m 2}}} \frac{\sqrt[4]{2\left(1-\Delta H_{P}\right)^{2}-1}}{1-\sqrt[4]{2\left(1-\Delta H_{P}\right)^{2}-1}}
$$

Substituting $R_{D}=\left(1 / g_{m 1}\right)+R s$ (the unity gain condition), $g_{m 2}$, and $C_{B}$ in eqn. 5.10 results the following pole and zero conditions:

$$
\begin{aligned}
& \left|f_{p, B}\right| \leq \frac{1}{2 \pi R_{D} C_{B}} \sqrt[2]{2\left(1-\Delta H_{P}\right)^{2}-1} \\
& \left|f_{z, B}\right| \leq \frac{1}{2 \pi R_{D} C_{B}}
\end{aligned}
$$

Again, eqn. 5.13a provides an estimate of the maximum possible pole frequency of the Buckwalter delay circuit, and eqn. 5.3 and 5.4 and Fig. 5.3 relate this to delay variation.

\subsection{Verification and design examples}

To verify analysis and exemplify how the analytical equations can be used during design, we will address two design questions as follows: 1) Which of the delay cells achieves the highest $f_{p}$ for a given power budget? and 2) What is the power consumption for each delay circuit provided that they fulfill the same delay and noise figure requirements?

We will use a CMOS process UMC 180 -nm supply voltage that is equal to $1.8 \mathrm{~V}$.

A. Maximum $\mathrm{f}_{\mathrm{p}}$ for a Given Power Budget

Suppose the design requirements for a delay cell in a cascaded chain are dc gain $>-1 \mathrm{~dB}$ and $\Delta \mathrm{Hp}<1 \mathrm{~dB}$, and we allow a maximum dc current of $3.5 \mathrm{~mA}$. Assume also two loading cases: $\mathrm{C}_{\mathrm{L}}$ is 0 or $2 \mathrm{pF}$. We aim at finding the best circuit w.r.t. delay variations and, hence, compare the maximum achievable $f_{p}$. To reduce the channel length 
Chapter 5. Frequency Limitations of First-Order gm-RC All-pass Delay Circuits

modulation in transistors, their lengths are chosen to be $240 \mathrm{~nm}$. The overdrive voltage of all transistors is chosen equal for both circuits $\left(\mathrm{V}_{\mathrm{GS}, \mathrm{OV}}=75 \mathrm{mV}\right)$, so that $\mathrm{f}_{\mathrm{T}}$ of the transistors is equal. For Buckwalter's cell, the size of $\mathrm{M}_{2}$ is chosen to be $\approx 15$ times of M1 to satisfy eqn. 5.11 , even for $R_{s}=0$ (see Table 5.1).

\begin{tabular}{|c|c|c|c|}
\hline Circuit & $\mathrm{W} / \mathrm{L}[\mu \mathrm{m} / \mu \mathrm{m}]$ & $\mathrm{V}_{\mathrm{GS}, \mathrm{OV}}[\mathrm{mV}]$ & $\mathrm{f}_{\mathrm{T}}[\mathrm{GHz}]$ \\
\hline "Classical" & $420.76 / 0.24$ & 75 & 12.4 \\
\hline Buckwalter & $(\mathrm{W} / \mathrm{L})_{1}=26.29 / 0.24$ & 75 & 12.4 \\
& $(\mathrm{~W} / \mathrm{L})_{2}=394.46 / 0.24$ & & \\
\hline
\end{tabular}

Table 5.1. Transistor design parameters used

For these transistor sizes, $\mathrm{C}_{\mathrm{gd}}$ for "Classical" is $173 \mathrm{fF}$, and $\mathrm{C}_{\mathrm{prs}}$ for "Buckwalter" is around $152 \mathrm{fF}$. Based on eqn. 5.8 and eqn. 5.12 and $C_{L}=0$, we find $C_{H} \geq 2434 \mathrm{fF}$ and $C_{B} \geq 943 \mathrm{fF}$, whereas for $C_{L}=2 p F$, we find $\mathrm{C}_{\mathrm{H}} \geq 16.508 \mathrm{pF}$ and $\mathrm{C}_{\mathrm{B}} \geq 1.771 \mathrm{pF}$. (In the Buckwalter circuit, the effect of $\mathrm{C}_{\mathrm{L}}$ is reduced by the buffer stage). The values for resistors calculated based on eqn. 5.7 are $R_{A}=R_{S}=170 \Omega$ and $R_{H}=252 \Omega$, whereas for the Buckwalter circuit, we find $R_{S}=0, R_{D}=249 \Omega$ (to have unity gain condition at dc: $R_{D}=R_{S}+1 / g_{m} 1$. Table 5.2 shows calculated and simulated values for each delay cell for the two loading conditions, which match good enough for first-cut circuit design. As noise and linearity are often also important, we also added simulation results for noise and IIP3 (with $50 \Omega$ as reference) for operating frequency $\approx f_{p}$, where $\Delta \mathrm{H}_{\mathrm{p}}$ has been estimated and verified. Overall, the Buckwalter cell achieves better higher $\mathrm{f}_{\mathrm{p}}$ and is less sensitive to $\mathrm{C}_{\mathrm{L}}$. 
Chapter 5. Frequency Limitations of First-Order gm-RC All-pass Delay Circuits

\begin{tabular}{|l|c|c|c|c|}
\hline & $\begin{array}{c}\text { Classic 0 } \\
\mathrm{fF}\end{array}$ & $\begin{array}{c}\text { Classic } \\
2 \mathrm{pF}\end{array}$ & $\begin{array}{c}\text { Buckw. } \\
0 \mathrm{fF}\end{array}$ & $\begin{array}{c}\text { Buckw. } \\
2 \mathrm{pF}\end{array}$ \\
\hline $\mathrm{C}_{\mathrm{L}}[\mathrm{pF}]$ & 0 & 2 & 0 & 2 \\
\hline $\mathrm{I}_{\mathrm{DC}}[\mathrm{mA}]$ & 3.5 & 3.5 & 3.5 & 3.5 \\
\hline$\#$ NMOS & 1 & 1 & 2 & 2 \\
\hline $\mathrm{f}_{\mathrm{T}, \mathrm{NMOS}}[\mathrm{GHz}]$ & 12.4 & 12.4 & 12.4 & 12.4 \\
\hline$\Delta \mathrm{H}_{\mathrm{p}}$ calc. $[\mathrm{dB}]$ & 1 & 1 & 1 & 1 \\
\hline$\Delta \mathrm{H}_{\mathrm{p}} \operatorname{sim} .[\mathrm{dB}]$ & 1.29 & 0.84 & 1.25 & 0.97 \\
\hline $\mathrm{A}_{\mathrm{vo}}$ calc. $[\mathrm{dB}]$ & -1 & -1 & 0 & 0 \\
\hline $\mathrm{A}_{\mathrm{vo}} \operatorname{sim} .[\mathrm{dB}]$ & -1.47 & -1.47 & -0.28 & -0.28 \\
\hline $\mathrm{f}_{\mathrm{p}}$ calc. $[\mathrm{MHz}]$ & 199 & 29.3 & 520 & 277 \\
\hline $\mathrm{f}_{\mathrm{p}} \operatorname{sim} .[\mathrm{MHz}]$ & 201 & 32.2 & 546 & 288 \\
\hline$\sqrt{\overline{V_{n, i n}}}{ }^{2}\left[\frac{n V}{\sqrt{H z}}\right]$ & 2.7 & 2.7 & 2.4 & 2.4 \\
\hline $\mathrm{IIP}_{3}[\mathrm{dBm}]$ & 16.5 & 17.5 & 14.8 & 17 \\
\hline
\end{tabular}

Table 5.2. Comparison of the delay cell properties.

Figs. 5.8 and 5.9 shows the simulated phase and gain plots of the delay cells. The values of $A_{v 0}$ (the dc gain) and $\Delta H p$ (gain drop at the pole frequency) are shown in the figures, where the pole frequency is defined as the frequency where the phase has dropped $90^{\circ}$ with respect to dc. Note that, although circuit parasitics affect the transfer function, the phase characteristic roughly resembles that of a firstorder all-pass filter. As predicted, the "Classical" circuit has attenuation at low frequency, but the Buckwalter circuit can have quite near to unity dc gain. Clearly, the Buckwalter circuits achieve the best frequency range, with also less overall attenuation. 
Chapter 5. Frequency Limitations of First-Order gm-RC All-pass Delay Circuits

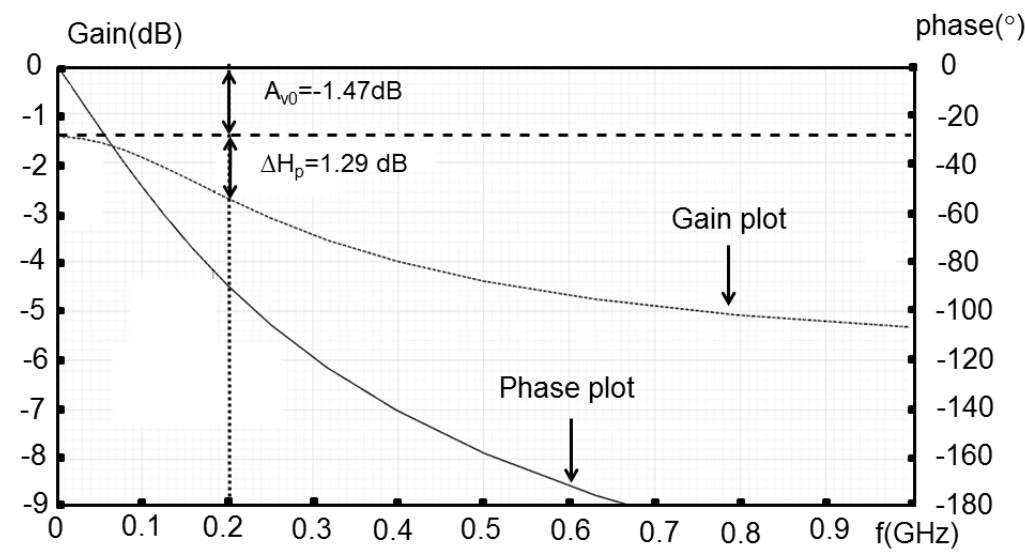

(a)

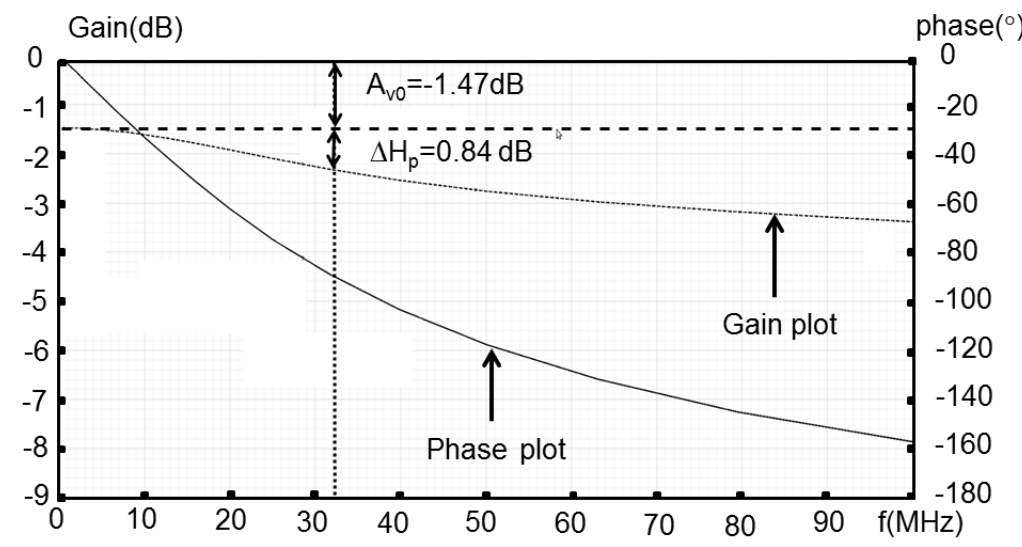

(b)

Fig. 5.8. Simulated phase and gain of "classical" delay cell for (a)

$$
\mathrm{C}_{\mathrm{L}}=0 \text { and (b) } \mathrm{C}_{\mathrm{L}}=2 \mathrm{pF} \text {. }
$$


Chapter 5. Frequency Limitations of First-Order gm-RC All-pass Delay Circuits

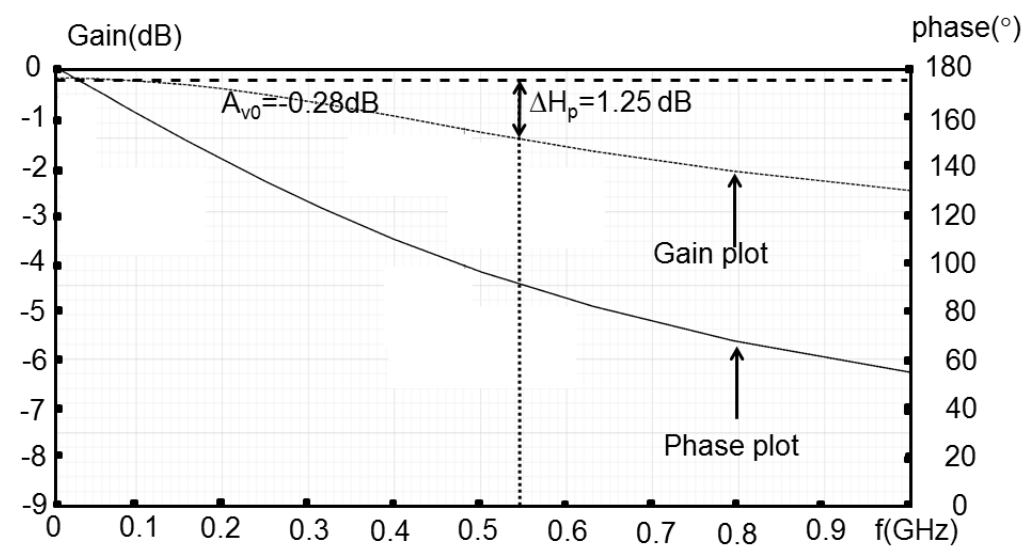

(a)

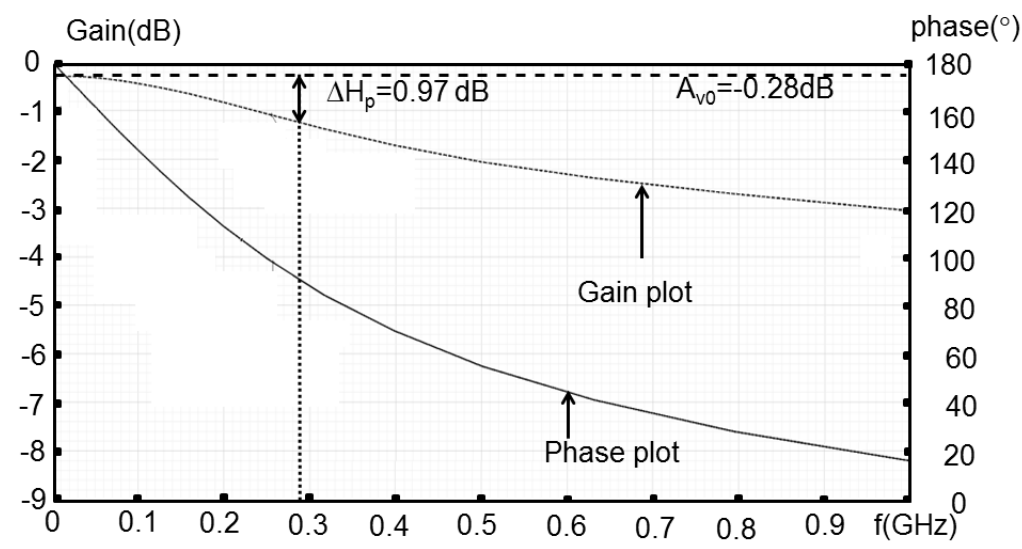

(b)

Fig. 5.9. Simulated phase and gain of "Buckwalter" delay cell for (a) $\mathrm{C}_{\mathrm{L}}=0$ and (b) $\mathrm{C}_{\mathrm{L}}=2 \mathrm{pF}$.

If we compare these results with that of a single-pole lowpass circuit, we achieve double the delay using an all-pass cell and about a 1-dB gain drop instead of $3 \mathrm{~dB}$ at the pole frequency. 


\section{B. Power Comparison for Equal Delay Cell Requirements}

Suppose now the following properties are desired: an operating frequency $\mathrm{f}_{0}=100 \mathrm{MHz}$, a relative delay variation of $5 \%$ for $\Delta \mathrm{f}= \pm 10$ $\mathrm{MHz}$ around $\mathrm{f}_{0}$, a dc gain of $>-1 \mathrm{~dB}, \Delta \mathrm{H}_{\mathrm{p}}<1 \mathrm{~dB}$, and $\mathrm{NF}=10 \mathrm{~dB}$ referred to $50 \Omega$, while we want to calculate the achievable delay per cell.

To obtain the maximum delay per cell, we choose the minimum $\mathrm{f}_{\mathrm{p}}$ that just satisfies eqn. 5.3 [8] for the aforementioned requirements. For design, suppose the initial sizes of the transistors are as in Table 5.2 , then from $f_{p}$, we find the value of capacitors. Using admittance scaling [10] to satisfy the noise figure requirements, we multiply all $\mathrm{W}$ and capacitor values by $\alpha$ and divide all resistor values by $\alpha$. With this method, the circuit delays do not change; however, the noise figure will change proportional to the power consumption (constant signalto-noise ratio/Power [10]). The power consumption also strongly depends on the overdrive voltage of the transistors. Substituting the delay variation of $5 \%$ and relative bandwidth of $\pm 10 \mathrm{MHz}$ into eqn. 5.3 results in $f_{\varphi=0} / f_{0}=-1$. In the graph in Fig. 5.3 , this corresponds to $\mathrm{f}_{0} / \mathrm{f}_{\mathrm{p}}=1.4$. Therefore, the minimum value of $\mathrm{f}_{\mathrm{p}}$ of the delay cells must be $100 / 1.4=71.4 \mathrm{MHz}$. This requires equal $\mathrm{f}_{\mathrm{p}}$ for both circuits, i.e., $\mathrm{R}_{\mathrm{H}} \mathrm{C}_{\mathrm{H}} \approx 1 / 2 \pi \mathrm{f}_{\mathrm{p}}=2.23 \mathrm{e}^{-9}$ and $\mathrm{R}_{\mathrm{D}} \mathrm{C}_{\mathrm{B}} \approx 1 / 2 \pi \mathrm{f}_{\mathrm{p}}=2.23 \mathrm{e}^{-9}$. To bring the noise figure to $10 \mathrm{~dB}$ for both, the values of resistors and capacitors will be $\mathrm{R}_{\mathrm{A}}=\mathrm{R}_{\mathrm{S}}=173.4 \Omega, \mathrm{R}_{\mathrm{H}}=257 \Omega, \mathrm{C}_{\mathrm{H}}=8.67 \mathrm{pF}$ and $\mathrm{R}_{\mathrm{S}}=0, \mathrm{R}_{\mathrm{D}}=321.2 \Omega, \mathrm{C}_{\mathrm{B}}=6.94$ pF. Table 5.3 shows the resulting aspect ratio and dc consumption of both circuits. The achievable delay with both circuits now is $3.025 \mathrm{~ns}$ at $\mathrm{f}_{0}=100 \mathrm{MHz}$. For the same noise figure, the Buckwalter cell consumes $20 \%$ less dc than the Classical circuit. In addition, the majority of its current is mainly consumed by the buffer transistor. For some applications, the loading capacitance may be small, and it might be possible to remove the buffer part of the Buckwalter circuit to reduce its power consumption. 
Chapter 5. Frequency Limitations of First-Order gm-RC All-pass Delay Circuits

\begin{tabular}{|c|c|c|c|c|c|}
\hline & $\begin{array}{c}\mathrm{W} / \mathrm{L} \\
{[\mu \mathrm{m} / \mu \mathrm{m}]}\end{array}$ & $\begin{array}{c}\mathrm{V}_{\text {ov }} \\
{[\mathrm{mV}]}\end{array}$ & $\begin{array}{c}\mathrm{f} \\
{[\mathrm{GHz}]}\end{array}$ & $\begin{array}{c}\mathrm{I}_{\text {bias }} \\
{[\mathrm{mA}]}\end{array}$ & $\begin{array}{c}\text { Total I } \\
{[\mathrm{mA}]}\end{array}$ \\
\hline "Classical" & $412.45 / 0.24$ & 75 & 12.4 & 3.43 & 3.43 \\
\hline "Buckwalter" & $20.37 / 0.24$ & 75 & 12.4 & 0.17 & 2.71 \\
\cline { 2 - 5 } & $305.78 / 0.24$ & 75 & 12.4 & 2.54 & \\
\hline
\end{tabular}

Table 5.3. Parameters for equal delay and 10-dB noise figure

\subsection{Summary and conclusion}

A method for analyzing the maximum useful frequency range of the first-order all-pass delay cells has been introduced. It has been used to analyze and compare two well-known $\mathrm{g}_{\mathrm{m}}-\mathrm{RC}$ delay cells, i.e., the "Classical" and the "Buckwalter" allpass cell. The analysis holds for single and cascaded identical cells. To this end, the cells have been analyzed as a self-loaded content with an arbitrary extra loading capacitance. The resulting transfer function deviates from ideal delaycell behavior in two key aspects, i.e., both the gain and delay are frequency dependent. Design boundary conditions were derived for each all-pass circuit to keep the gain variation below a specified maximum and ensure that the shape of the transfer function still resembles the simple $1^{\text {st }}$ order all-pass function (eqn. 5.1), which is characterized by one pole frequency $f_{p}$. The design boundary conditions were then expressed as constraints on the maximum achievable pole frequency $f_{p}$. Substituting $f_{p}$ in eqn. 5.4 , eqn. 5.3 can now be used to estimate the amount of delay variation as a function of the frequency variation $\Delta \mathrm{f}$ around the nominal operating frequency $\mathrm{f}_{0}$.

For any single $1^{\text {st }}$ order all-pass delay cell, or approximation thereof, a larger value of $f_{p}$ renders smaller delay variations over a given frequency range $\Delta \mathrm{f}$ around $\mathrm{f}_{0}$, but also smaller delay (see Fig. 5.3). When cascading multiple cells, each cell needs to realize less delay, and hence can have higher $\mathrm{f}_{\mathrm{p}}$, resulting in less relative delay variations (eqn. 5.3). This thus results in a larger useful frequency range (but more chip area and power consumption). The equations in this chapter model the trade-offs between delay-cell pole frequency $f_{p}$, center frequency $f_{0}$, frequency range $\Delta f$ and delay variation, while 
keeping amplitude variations within a budget and allowing for improving performance by cascading cells.

To exemplify the usefulness of the analysis, it was applied to two delay cells to compare their relative performance and estimate their delay variation versus frequency. With the help of the derived design equations, an optimization of $\mathrm{f}_{\mathrm{p}}$ was illustrated, keeping delay and power constraints fixed, comparing results with and without extra capacitance. Secondly, the analysis results were exploited to achieve equal delay with different circuits, under the condition of fixed noise performance, while comparing power dissipations. Similar analysis can be done for other kinds of $1^{\text {st }}$ order $\mathrm{g}_{\mathrm{m}}$-RC all-pass delay cells.

\section{References}

[1] T. -S. Chu, J. Roderick, and H. Hashemi, "An integrated ultrawideband timed array receiver in $0.13 \mu \mathrm{m}$ CMOS using a path-sharing true time delay architecture," IEEE J. Solid-State Circuits, vol. 42, no. 12, pp. 2834- 2850, Dec. 2007.

[2] J. Buckwalter and A. Hajimiri, "An active analog delay and the delay reference loop," in Proc. IEEE RFIC Symp., 2004, pp. 17-20.

[3] R. J. Mailloux, Phased Array Antenna Handbook. Hoboken, NJ, USA: Wiley, 2005.

[4] S. K. Garakoui, E. A. M. Klumperink, B. Nauta, and F. E. van Vliet, "Phased-array antenna beam squinting related to frequency dependency of delay circuits," in Proc. Eur. Radar Conf., 2011, pp. 1304-1307.

[5] D. Pozar, Microwave Engineering. Hoboken, NJ, USA: Wiley, 2005.

[6] P. Horowitz, The Art of Electronics. Cambridge, U.K.: Cambridge Univ. Press, 1980. 
Chapter 5. Frequency Limitations of First-Order gm-RC All-pass Delay Circuits

[7] K. Bult and H. Wallinga, "A CMOS analog continuous-time delay line with adaptive delay-time control," IEEE J. Solid-State Circuits, vol. 23, no. 3, pp. 759-766, Jun. 1988.

[8] S. K. Garakoui, E. A.M. Klumperink, B. Nauta, and F. E. van Vliet, "Time delay circuits: A quality criterion for delay variations versus frequency," in Proc. IEEE ISCAS, 2010, pp. 4281-4284.

[9] R. B. Dome, "Wideband audio phase-shift networks," Electronics, vol. 19, pp. 112-115, Dec. 1946.

[10] E. A. M. Klumperink and B. Nauta, "Systematic comparison of HF CMOS transconductors," IEEE Trans. Circuits Syst. II. Analog Digital Signal Process., vol. 50, no. 10, pp. 728-741, Oct. 2003. 


\section{Chapter 6}

\section{Compact Cascadable $\mathrm{g}_{\mathrm{m}}-\mathrm{C}$ All-Pass True Time Delay Cell with Reduced Delay Variation over Frequency ${ }^{1}$}

\subsection{Introduction}

TIME delay circuits have broad applications in communication systems, e.g. for FIR and IIR filters [1], equalizers [2], and wide band beam forming [3], [4]. This chapter deals with the latter application, where a "timed array" is targeted instead of the more commonly used phased array. In a timed array, true time delays are used instead of the narrowband phase shifter approximation. In this way beam squinting can be minimized [4], [5]. In beam forming receivers the variable delay cells compensate the relative delay of signals of the antenna channels. The transfer function of an ideal delay cell is: $H(s)=e^{-s^{\tau}}$ (Fig. 6.1). Its gain is 1 and its phase is linear versus frequency. The delay $(\tau)$ at frequency $f_{0}$ is: $\tau\left(f_{0}\right)=-\varphi\left(f_{0}\right) / 2 \pi f_{0}$, ideally independent of $f_{0}$ (linear phase). Note that we consider true time delay here, not group delay, which is defined as $-\partial \varphi / \partial \omega$. Achieving constant true time delay is tougher as it not only requires constant group delay independent of

1 This chapter is an exact copy of the following paper:

S. K. Garakoui, E. A. M. Klumperink, B. Nauta and F. E. van Vliet, " Compact Cascadable gm-C All-Pass True Time Delay Cell With Reduced Delay Variation Over Frequency," IEEE Journal of Solid-State Circuits, vol. 50, no. 3, pp. 693-703, March. 2015. 
Chapter 6. Compact Cascadable gm-C All-Pass True Time Delay Cell with Reduced Delay Variation over Frequency

frequency but also a constant ratio between $-\varphi$ and $\omega$ independent of frequency [6].
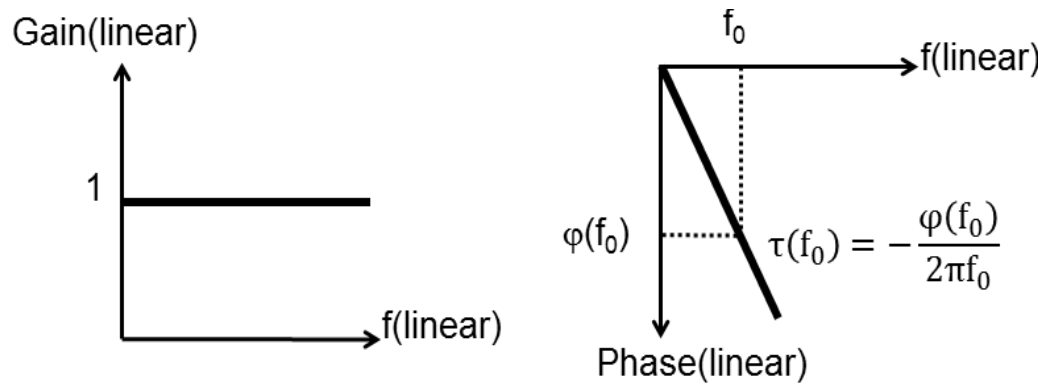

Fig. 6.1. The gain and phase transfer function of an ideal time delay cell

There are different IC compatible circuits to approximate a time delay, e.g. transmission lines [7], [8], LC delay lines [9], switched capacitor delay circuits [10] and $g_{m}-R C$ or $g_{m}-C$ all-pass filters [10]. However, at low GHz frequencies, transmission lines and LC delay lines in CMOS are unpractical due to the low quality factor of coils, loss of the transmission lines and their large sizes. Switched capacitor time-delay circuits on the other hand are not fast enough for low $\mathrm{GHz}$ applications. One of the few remaining options is to exploit an all-pass filter approximation of a delay, e.g. a $1^{\text {st }}$ order all-pass filter:

$H_{a p_{1}}(s)=\frac{1-s(\tau / 2)}{1+s(\tau / 2)}$

The transfer function of this all-pass filter is plotted in Fig 6.2. At low frequencies it approximates the ideal delay cell but at higher frequencies the delay is reduced and delay variations occur. This delay variation is quantified via the criterion $f_{\varphi=0}$ [6] which is the crossing point of the frequency axis and the tangent to the phase curve at operating frequency $f_{0}$ (Fig. 6.2). 
Chapter 6. Compact Cascadable gm-C All-Pass True Time Delay Cell with Reduced Delay Variation over Frequency
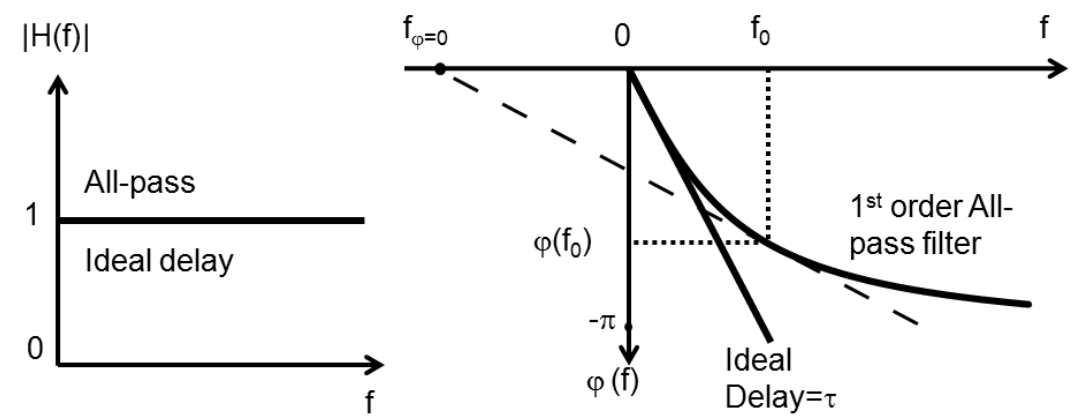

Fig. 6.2. 1st order all-pass filter with extrapolation point $f_{\varphi=0}$ vs. ideal delay (linear scales)

The delay variation in $\pm \Delta \mathrm{f}$ around $\mathrm{f}_{0}$ is approximately:

$$
\frac{\tau\left(f_{0} \pm \Delta f\right)-\tau\left(f_{0}\right)}{\tau\left(f_{0}\right)} \approx \frac{\frac{f_{\varphi=0}}{f_{0}}}{1-\frac{f_{\varphi=0}}{f_{0}}} \cdot \frac{ \pm \Delta f}{f_{0}}
$$

The 1st order all-pass transfer function can be realized both with $\mathrm{g}_{\mathrm{m}}$-RC filters [2], [11] (see Fig. 6.3) and the $\mathrm{g}_{\mathrm{m}}-\mathrm{C}$ filter presented in this chapter and in [12]. 


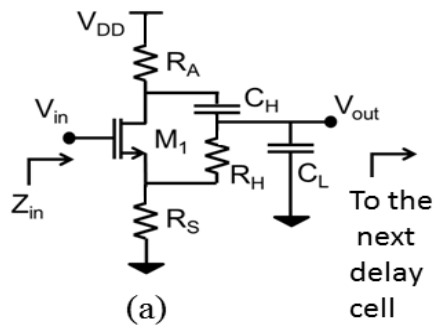

(a)

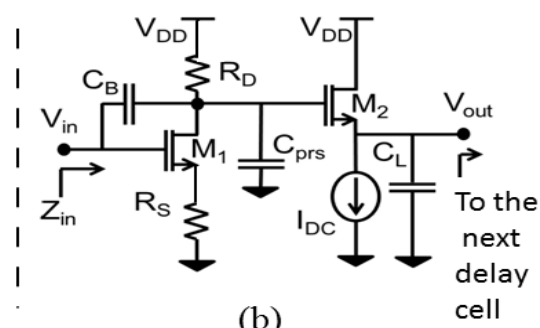

(b)

Fig. 6.3. Two known $g_{m}-R C$ delay circuits: a) of [11] and b) [2]

In [13] a benchmarking method has been proposed to compare delay cells based on $f_{\varphi=0}$. This method is re-used here to show that the $\mathrm{g}_{\mathrm{m}}-\mathrm{C}$ delay cell of [12] has better performance than other published designs. Moreover, the feasibility of a compact broadband beamforming IC with $\mathrm{g}_{\mathrm{m}} \mathrm{C}$ delay cells is demonstrated. Apart from bandwidth, other important properties of the delay cell are: 1) Cascadability; 2) Compactness; 3) Wide delay tuning range; 4) High delay tuning resolution and precision; 5) Gain controllability; 6) Noise figure and 7) Linearity. In [12] it has been shown that the $\mathrm{g}_{\mathrm{m}}-\mathrm{RC}$ allpass filters of [2] and [11] (shown in Fig. 6.3) do not work adequately up to several GHz in UMC $180 \mathrm{~nm}$ CMOS because of their high parasitic capacitors. Besides, they need DC blocking capacitors or sourcefollower buffer circuits to realize cascadability, which limits the bandwidth and/or results in high current consumption. It will be proven that the $\mathrm{g}_{\mathrm{m}}-\mathrm{C}$ topology of [12] has better performance: 1) Low delay variation over a $5 \mathrm{x}$ wider frequency band compared to other reported $\mathrm{g}_{\mathrm{m}} \mathrm{RC}$ delay circuits, while maintain similar noise and nonlinearity performance; 2) Compactness compared to LC or transmission lines; 3) High resolution of delay and gain tuning; 4) Direct cascadability. Compared to [12], this chapter adds circuit analysis and circuit optimization techniques, e.g. for phase linearization and bandwidth extension. The structure of this paper is as follows: in section 6.2, the all-pass filter of [12] is explained. Section 6.3 assesses non-idealities of the delay cell while section 6.4 discusses improvements of its characteristics. Section 6.5 establishes a relation between requirements of the beam forming system and the delay cell. 
Chapter 6. Compact Cascadable gm-C All-Pass True Time Delay Cell with Reduced Delay Variation over Frequency

Section 6.6 presents the sub-circuits of the timed array IC. Section 6.7 is the chip implementation and measurement results, while section 6.8 draws conclusions.

\section{$6.2 \quad 1^{\text {st }}$ order all-pass delay cell}

The transfer function of the $1^{\text {st }}$ order all-pass filter of (6.1) can be re-written as a combination of a low-pass part with DC-gain of two and a unity-gain part [14] as:

$H_{a p_{1}}(s)=\frac{1-s(\tau / 2)}{1+s(\tau / 2)}=\frac{2}{1+s(\tau / 2)}-1$

It is realizable without floating capacitors and hence with good bandwidth potential, because the low-pass part can be implemented by a capacitor to ground and the unity gain part does not require capacitors. Fig. 6.4 shows the block level and $g_{m}-R C$ implementation of this all-pass filter.

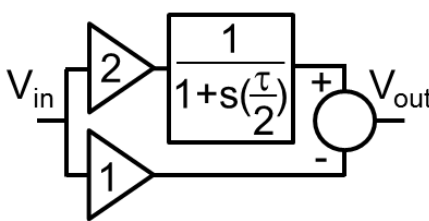

Block view

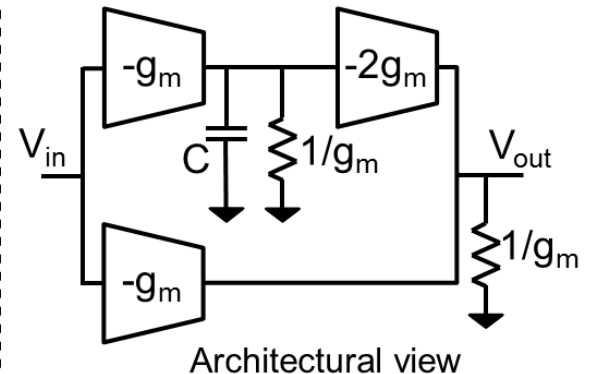

Architectural view

Fig. 6.4. The block view and architectural view of the 1st order all-pass filter implementable with no floating caps

Aiming for direct cascadability, the $\mathrm{g}_{\mathrm{m}}-\mathrm{C}$ topology of Fig. 6.5 [12] with equal input and output DC voltages was proposed. Transistors $M_{1}$, $M_{4}, M_{5}$ and $M_{3}$ realize the low-pass signal path with a DC-gain of 2 . Transistors $M_{2}$ and $M_{3}$ realize the inverting unity gain path. Using PMOS transistors in the "slow low-pass path" and faster NMOS 
Chapter 6. Compact Cascadable gm-C All-Pass True Time Delay Cell with Reduced Delay Variation over Frequency

transistor in the unity gain path, the useful frequency range of the delay cell is maximized. Also, current re-use for NMOS and PMOS transistors reduces power consumption. The DC input voltage $V_{\text {in,DC }}$ results in equal drain currents $I_{D C}$ in $M_{1}, M_{2}, M_{3}$ and $M_{4}$, and 2I $I_{D C}$ for $M_{5}$. Therefore, $\mathrm{V}_{\text {out }, \mathrm{DC}}=\mathrm{V}_{\mathrm{in}, \mathrm{DC}}$.

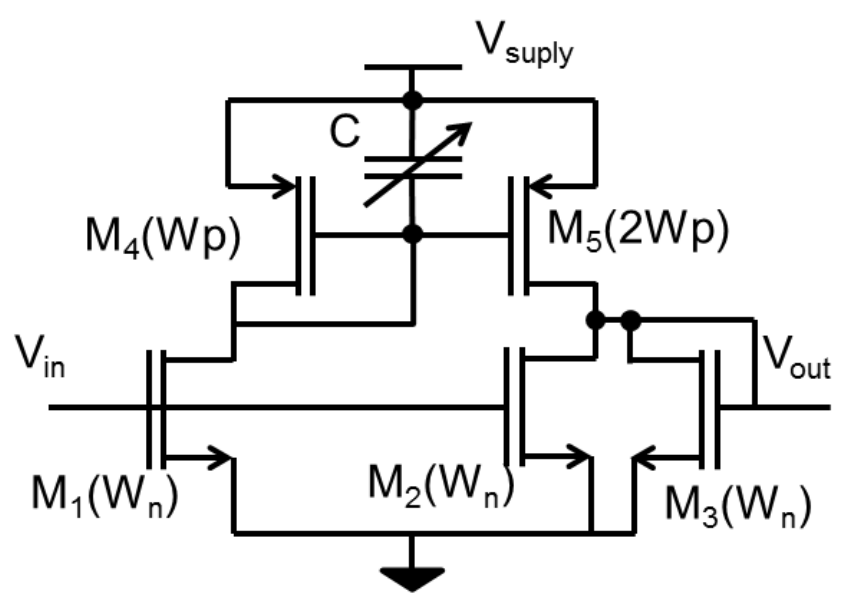

Fig. 6.5. The proposed $1^{\text {st }}$ order $g_{m}-C$ all-pass filter in [12].

Modelling $\mathrm{M}_{4}$ by its small-signal $\mathrm{g}_{\mathrm{m} 4}$ and $\mathrm{C}$ as the total capacitance, the transfer function and its low frequency delay can be written as:

$H(s)=\frac{V_{\text {out }}(s)}{V_{\text {in }}(s)}=\frac{1-\frac{s C}{g_{m 4}}}{1+\frac{s C}{g_{m 4}}}$

$\tau_{L F} \approx 2 \frac{C}{g_{m 4}}$

The low-frequency delay is made controllable by splitting $\mathrm{C}$ in switchable binary weighted capacitors. Fig. 6.6 shows the bias circuit of the first delay cell of a delay line. It is the only cell with an ACcoupling capacitor to the input $\mathrm{RF}$ signal, $\mathrm{V}_{\mathrm{in}, \mathrm{RF}}$. As each signal path has 
Chapter 6. Compact Cascadable gm-C All-Pass True Time Delay Cell with Reduced Delay Variation over Frequency

this, no difference in gain and delay results. The DC voltage of $\mathrm{V}_{\text {out }}$ is equal to $V_{D C \text {,bias. }} R_{B}$ is made more than 10 times larger than the source impedance of $\mathrm{V}_{\mathrm{in}, \mathrm{RF}}$, for insignificant attenuation.

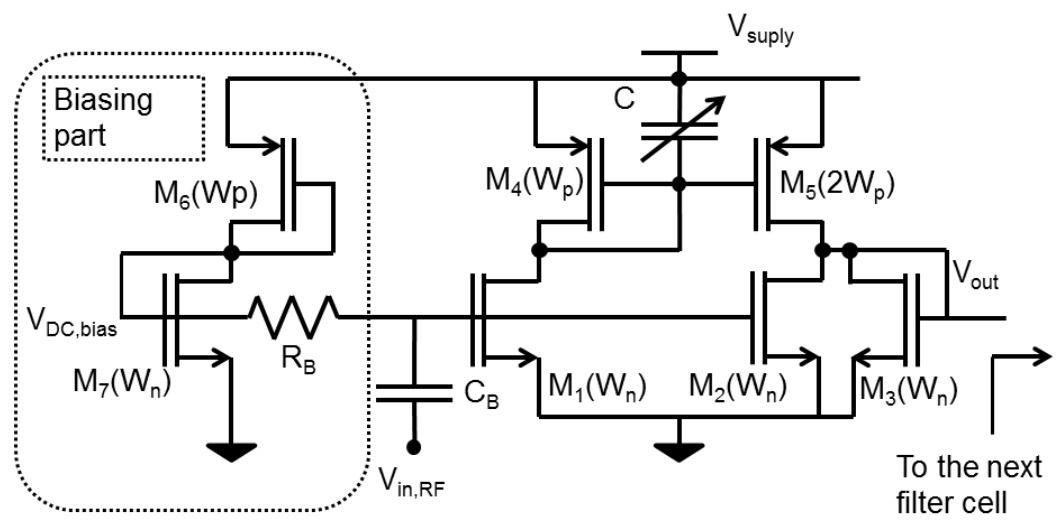

Fig. 6.6: The biasing circuitry of the first filter cell in a cascade line

\subsection{The non-idealities of the delay cell}

As the aim is to cascade cells, the non-idealities of a delay cell will now be analyzed with a capacitive load equal to the input capacitance of the next delay cell: $\mathrm{C}_{\mathrm{gs}, \mathrm{M} 1}+\mathrm{C}_{\mathrm{gs}, \mathrm{M} 2}$. In the analysis the effect of $\mathrm{C}_{\mathrm{gd}}$ will be neglected as the voltage gain is low (unity gain all-pass behavior), while the right half-plane zero introduced by at $\mathrm{g}_{\mathrm{m}} / \mathrm{C}_{\mathrm{gd}}$ is in the range of $50 \mathrm{GHz}$ for the transistors used. This is well beyond the targeting low-GHz operating frequency, and therefore for the sake of simplicity we neglected its effect. This assumption was validated by checking hand calculation versus simulation results.

A. Finite output impedance and parasitic capacitances

Considering finite output impedances of the transistors and the parasitic capacitors which affect the pole/zero frequency and DC gain, the transfer function of the filter becomes: 


$$
\begin{aligned}
& H(s) \\
& =\frac{1}{1+\frac{2}{g_{m n}}\left(g_{d s n}+g_{d s p}\right)} \cdot \frac{1-\frac{1}{g_{m p}}\left(g_{d s n}+g_{d s p}\right)}{1+\frac{1}{g_{m p}}\left(g_{d s n}+g_{d s p}\right)} \cdot \frac{1-\frac{s C}{\left[g_{m p}-\left(g_{d s n}+g_{d s p}\right)\right]}}{1+\frac{s C}{\left[g_{m p}-\left(g_{d s n}+g_{d s p}\right)\right]}} \\
& \cdot \frac{1}{1+\frac{s C_{L}}{\left[g_{m n}+2\left(g_{d s n}+g_{d s p}\right)\right]}}
\end{aligned}
$$

Where $g_{m n}$ and $g_{d s n}$ are the transconductance and output conductance of $M_{1}, M_{2}$ and $M_{3}$ in saturation, $g_{m p}$ and $g_{d s p}$ those of $M_{4}$ and $2 \mathrm{~g}_{\mathrm{mp}}$ and $2 \mathrm{~g}_{\mathrm{dsp}}$ of $\mathrm{M}_{5}$. The parasitic capacitances $\mathrm{C}_{\mathrm{gs}, \mathrm{M} 4}, \mathrm{C}_{\mathrm{gs}, \mathrm{M} 5}, \mathrm{C}_{\mathrm{db}, \mathrm{M} 4}$ and $\mathrm{C}_{\mathrm{db}, \mathrm{M} 3}$ are absorbed in $\mathrm{C}$. Also $\mathrm{C}_{\mathrm{L}}$ absorbs the parasitic capacitors $\mathrm{C}_{\mathrm{gs}, \mathrm{M} 3}, \mathrm{C}_{\mathrm{db}, \mathrm{M} 5}, \mathrm{C}_{\mathrm{db}, \mathrm{M} 2}, \mathrm{C}_{\mathrm{db}, \mathrm{M} 3}$ plus the input capacitance of the next delay cell $\left(\mathrm{C}_{\mathrm{gs}, \mathrm{M} 1}+\mathrm{C}_{\mathrm{gs}, \mathrm{M} 2}\right)$. The transfer function (6) deviates from the ideal one (4) in two aspects: 1) the DC-gain is less than unity; and 2) an extra high frequency pole causes both an extra phase shift and a high frequency gain roll-off. If the following conditions are satisfied:

$$
\begin{aligned}
& g_{m n} \gg 2\left(g_{d s n}+g_{d s p}\right) \\
& g_{m p} \gg 2\left(g_{d s n}+g_{d s p}\right)
\end{aligned}
$$

Then the transfer function can be rewritten as:

$$
H(s)=\frac{1-\frac{2}{g_{m p}}\left(g_{d s n}+g_{d s p}\right)}{1+\frac{2}{g_{m n}}\left(g_{d s n}+g_{d s p}\right)} \cdot \frac{1-\frac{s C}{g_{m p}}}{1+\frac{s C}{g_{m p}}} \cdot \frac{1}{1+\frac{s C_{L}}{g_{m n}}}
$$

Using the analysis in [13], the maximum usable pole frequency $f_{p}$ is defined as the frequency where the gain roll-off with respect to DC is less than $\Delta \mathrm{H}_{\mathrm{p}}$, resulting in:

$$
f_{p} \leq \frac{g_{m n}}{2 \pi C_{L}} \sqrt{\frac{1}{\left(1-\Delta H_{p}\right)^{2}}-1}
$$


Chapter 6. Compact Cascadable gm-C All-Pass True Time Delay Cell with Reduced Delay Variation over Frequency

For frequencies larger than $f_{p}$, the roll-off is more than $\Delta H_{p}$. Substituting $\mathrm{C}_{\mathrm{L}} \approx \mathrm{C}_{\mathrm{gs}, \mathrm{M} 1}+\mathrm{C}_{\mathrm{gs}, \mathrm{M} 2}+\mathrm{C}_{\mathrm{gs}, \mathrm{M} 3}=3 \mathrm{C}_{\mathrm{gs}, \mathrm{M} 1}$ and $\mathrm{f}_{\mathrm{t}, \mathrm{M} 1} \approx \mathrm{g}_{\mathrm{mn}} / 2 \pi \mathrm{C}_{\mathrm{gs}, \mathrm{M} 1}$ (unity current gain) in (6.9) results in:

$f_{p} \leq \frac{f_{t, M 1}}{3} \sqrt{\frac{1}{\left(1-\Delta H_{p}\right)^{2}}-1}$

To estimate $\mathrm{f}_{\mathrm{p}}$ and compare it with the delay cells reported in [2] and [11] (benchmarked in [13]), the same technology (UMC 180nm CMOS), same $\Delta \mathrm{H}_{\mathrm{p}}=1 \mathrm{~dB}$ and same $\mathrm{f}_{\mathrm{t}, \mathrm{M} 1}=12.4 \mathrm{GHz}$ for the NMOS transistors have been used. The choice $\Delta \mathrm{H}_{\mathrm{p}}=1 \mathrm{~dB}$ is only for comparison to [13], and it will be reduced in section 6.4 where several delay cells will be cascaded. Substituting the values in (6.10), the result is $\mathrm{f}_{\mathrm{p}} \leq 2 \mathrm{GHz}$, which is a $4 \mathrm{x}$ improvement compared to other circuits in [13]. Fig. 6.7 shows the simulation results of the delay cell. Reading $\mathrm{f}_{\mathrm{p}}$ as the frequency where $45^{\circ}$ phase shift occurs w.r.t. DC, we find $\mathrm{f}_{\mathrm{p}} \approx 1.7 \mathrm{GHz}$ and a gain roll-off of $\approx 1.5 \mathrm{~dB}$ at $\mathrm{f}_{\mathrm{p}}$ which is due to the parasitic capacitor effects at the output of the cell. Also the DC gain is not $0 \mathrm{~dB}$ due to the finite output impedances of transistors. In section 6.4 the DC-gain will be calibrated to $0 \mathrm{~dB}$ and the useful frequency range is increased further to $5 \mathrm{x}$ (up to $2.5 \mathrm{GHz}$ ) that of other reported $\mathrm{g}_{\mathrm{m}}-\mathrm{RC}$ all-pass delay cells. 
Chapter 6. Compact Cascadable gm-C All-Pass True Time Delay Cell with Reduced Delay Variation over Frequency

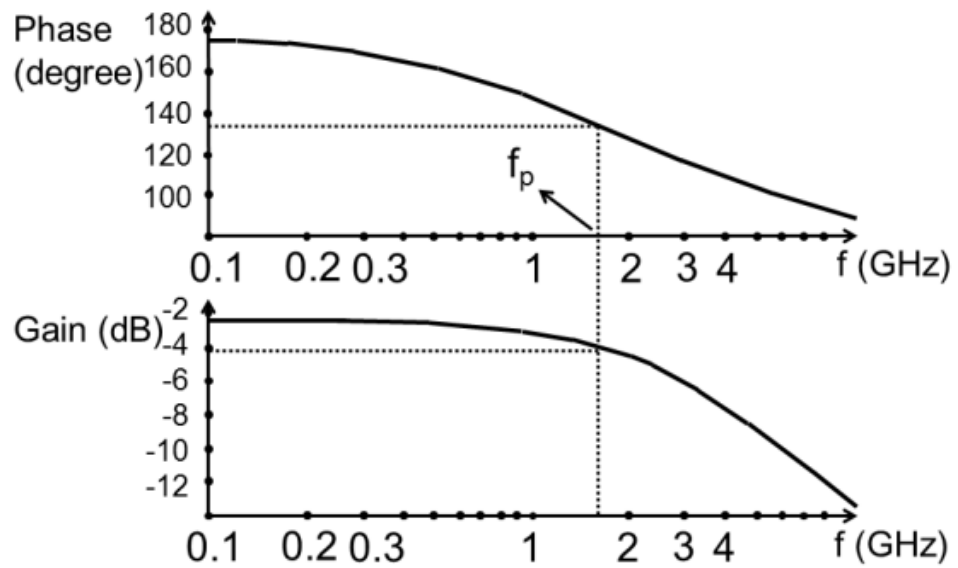

Fig. 6.7. The phase at the gate of $\mathrm{M}_{5}$, and the output of the delay cell in a delay line

The operating bandwidth is limited to $f_{p}$, to keep the gain roll-off less than $\Delta \mathrm{H}_{\mathrm{p}}$. Within the operating bandwidth, the value of the true time delay and group delay mainly depends on $f_{p}$, but may also be affected by the $-3 \mathrm{~dB}$ gain-roll-off frequency $\mathrm{f}_{-3 \mathrm{~dB}}$ due to the parasitic pole at the output. Because $f_{-3 d B}\left(\approx f_{t, M} / 3\right)$ is much larger than $f_{p}$, a linear phase approximation can be made. This causes both a constant time delay shift and group delay shift equal to $1 / 2 \pi \mathrm{f}_{-3 \mathrm{db}}$. Eqn. 6.11 shows expressions for both the total true time delay $(\tau)$ and group delay $\left(\tau_{\mathrm{g}}\right)$ of the delay cell:

$$
\begin{gathered}
\tau \approx \frac{2 \tan ^{-1} \frac{f}{f_{p}}}{2 \pi f}+\frac{1}{2 \pi f_{-3 d B}} \\
\tau_{g} \approx \frac{f_{p}}{\pi\left(f^{2}+f_{p}^{2}\right)}+\frac{1}{2 \pi f_{-3 d B}}
\end{gathered}
$$


Chapter 6. Compact Cascadable gm-C All-Pass True Time Delay Cell with Reduced Delay Variation over Frequency

In both equations the second term is much smaller than the first term.

\section{B. Nonlinearity}

The nonlinear V-to-I conversions in $\mathrm{M}_{1}$ and $\mathrm{M}_{2}$ can be compensated by the I-to- $V$ function of $\mathrm{M}_{3}$, which are inverse functions. Also, the mirror $\mathrm{M}_{4}$ and $\mathrm{M}_{5}$ with gain 2, ideally renders an inverse function nonlinearity compensation. However, reactive harmonic distortion [15] occurs at frequencies well below the pole frequency. The I-V conversion by $\mathrm{M}_{4}$ becomes more linear for higher frequencies, as the (linear) capacitor starts to dominate the I-V conversion instead of the square-root I-V function due to diode connected transistor $\mathrm{M}_{4}$. As the $\mathrm{V}-\mathrm{I}$ conversion of $\mathrm{M}_{5}$ remains non-linear (quadratic for long transistors), the overall function is nonlinear. Due to the phase shifts caused by capacitor $C$, the non-linearity compensation between $M_{1}, M_{2}$ and $\mathrm{M}_{3}$ is degraded. Therefore, the nonlinearity of the filters cell increases by increasing the frequency.

C. Thermal noise

The input referred thermal noise of the delay cell can be written as:

$$
\begin{aligned}
& \overline{v^{2}{ }_{l n}}=8 k T \gamma\left(\frac{g_{m n}+g_{m p}}{g_{m n}{ }^{2}}\right)\left[\frac{3 g_{m p}{ }^{2}+(C \omega)^{2}}{g_{m p}{ }^{2}+(C \omega)^{2}}\right] \\
& =8 k T \gamma\left(\frac{g_{m n}+g_{m p}}{g_{m n}{ }^{2}}\right)\left[\frac{3\left(\frac{f_{p}}{f}\right)^{2}+1}{\left(\frac{f_{p}}{f}\right)^{2}+1}\right]
\end{aligned}
$$

where $\gamma$ is the noise excess factor of a MOSFET. As (6.12) shows, the input referred noise is frequency-dependent. In a delay line of $n$ cascaded delay cells with unity gain, the total input referred noise power is $\mathrm{n}$ times the noise of each individual delay cell. Therefore, in systems with variable numbers of cascaded delay cells, the total noise figure will be delay dependent. 
Chapter 6. Compact Cascadable gm-C All-Pass True Time Delay Cell with Reduced Delay Variation over Frequency

D. PVT sensitivity

Process, Voltage and Temperature (PVT) variations may affect the gain and amount of the delay of each delay cell. Due to mismatch and the finite output impedance of the transistors, the DC gain of the delay cell is not exactly one. In cascaded cells these errors add-up. A tuning mechanism for DC gains is addressed in section 6.4. Moreover, just as for any $\mathrm{g}_{\mathrm{m}}-\mathrm{C}$ filters, there will be spread in the filter time-constant and hence delay due to PVT. Using master-slave techniques [16], these variations can be cancelled largely, e.g. by using replicas of the delay cell in an oscillator loop, and tuning its frequency equal to a wellknown reference frequency.

\subsection{Delay cell enhancements}

We will now describe some techniques to reduce true time delay variation (make the delay more constant over the frequency band), extend bandwidth and (fine-) tune the delay and gain.

A. Phase linearization (small delay variations)

It is shown below that adding a resistor $\mathrm{R}$ between gate and drain of $\mathrm{M}_{4}$ (Fig. 6.8) improves the linearity of the phase transfer function in a limited frequency band. 


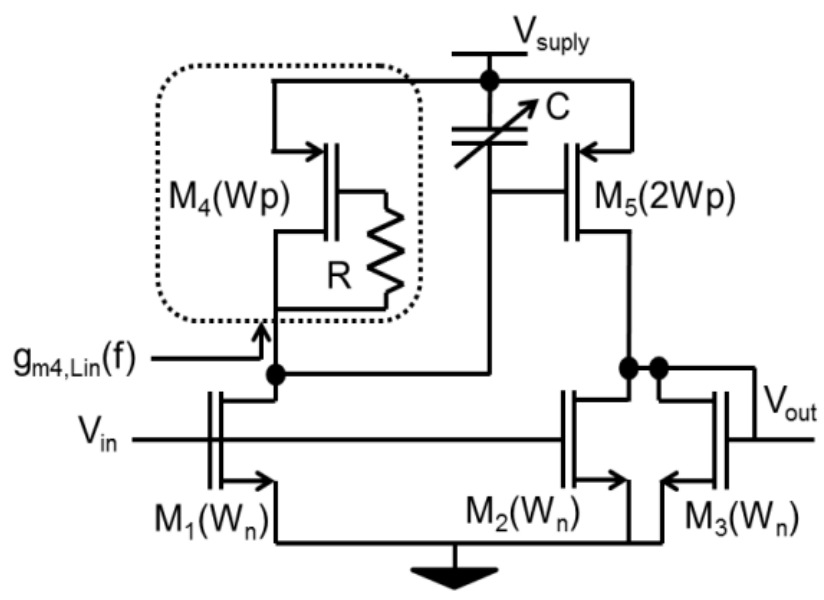

Fig. 6.8. The phase linearization technique

This can be considered as "inductive peaking" that is often used in wideband amplifiers for equalization of the gain [17]. Here, its purpose and optimization targets phase linearity and low $f_{\varphi=0}$, to minimize delay variation. The conductance $g_{m 4, L i n}$ of the linearizedphase circuit inside the dashed rectangle in Fig. 6.8 is:

$g_{m 4, L i n}(s)=g_{m 4} \cdot \frac{\frac{s C_{g s M 4}}{g_{m 4}}+1}{s R C_{g s M 4}+1}$

Its value for very low and very high frequencies is $\mathrm{g}_{\mathrm{m}, \mathrm{LF}} \approx \mathrm{g}_{\mathrm{m} 4}$ and $\mathrm{g}_{\mathrm{m}, \mathrm{HF}} \approx 1 / \mathrm{R}$ respectively. As is shown conceptually in Fig. 6.9, the phase transfer function of the linearized delay cell $\psi_{\text {lin }}$ shows a smaller value of $\mathrm{f}_{\varphi=0}$ compared to two other phase transfer functions $\psi_{1}$ and $\psi_{2}$. Hence not only the variation in group delay is reduced but also the variation in true time delay (low $\mathrm{f}_{\varphi=0}$ ). This happens for a certain optimum value of R. For low frequencies the phase curve is similar to that of an all-pass cell with its pole/zero at $\pm \mathrm{g}_{\mathrm{m}} / 2 \pi \mathrm{C}$ (curve $\psi_{1}$ ), while for high frequencies it follows the phase curve of a cell with pole/zero at $\pm 1 / 2 \pi \mathrm{RC}$ (curve $\psi_{2}$ ). For intermediate frequencies the phase curve is an interpolation between the two lines $\psi_{1}$ and $\psi_{2}$. A proper value of 
Chapter 6. Compact Cascadable gm-C All-Pass True Time Delay Cell with Reduced Delay Variation over Frequency

$\mathrm{R}$ found through parametric simulations, results in a curve $\left(\psi_{\mathrm{Lin}}\right)$ with minimum amount of the delay variations in a band $\pm \Delta f$ around $f_{0}$, i.e. a minimum value of the criterion $\mathrm{f}_{\varphi=0}$ (see eqn. (6.2)) [6], [5]. Note that closer proximity of $\mathrm{f}_{\varphi=0}$ to zero corresponds to less delay variation vs. frequency

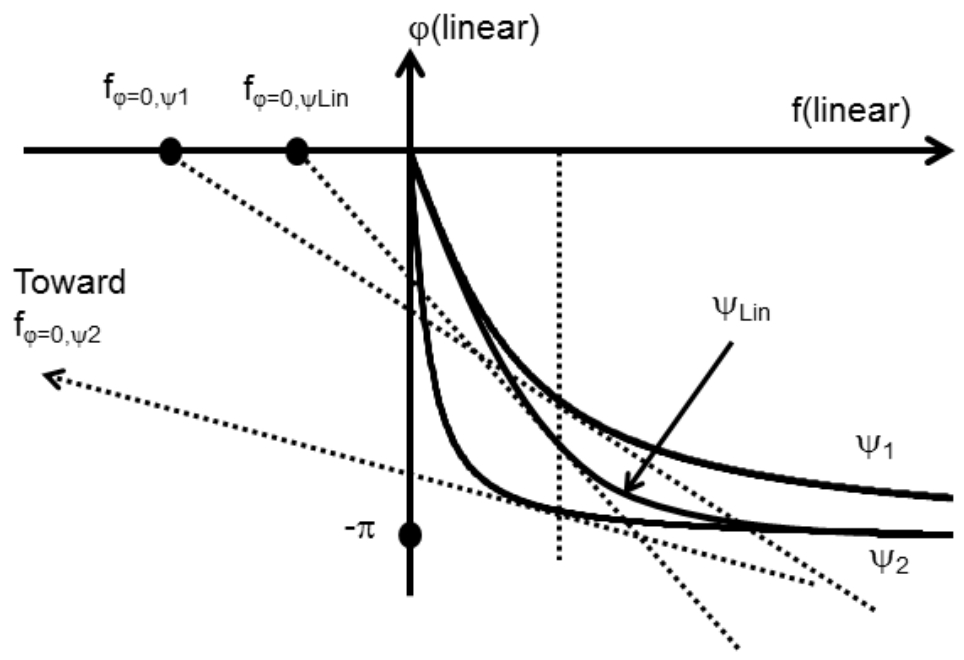

Fig. 6.9. Low frequency linearization technique of the phase transfer curve of the filter (conceptually depicted)

Fig. 6.10 shows simulated phase curves with $\mathrm{R}$ as parameter. The process technology used is now 160nm CMOS and Table 6.1 lists the circuit parameters.

\begin{tabular}{|c|c|c|c|c|c|}
\hline & $\mathrm{W} / \mathrm{L}(\mu \mathrm{m} / \mu \mathrm{m})$ & $\mathrm{V}_{\text {th }}(\mathrm{V})$ & $\mathrm{I}_{\mathrm{D}}(\mu \mathrm{A})$ & $\mathrm{V}_{\mathrm{GS}}(\mathrm{V})$ & $\mathrm{V}_{\mathrm{DS}}(\mathrm{V})$ \\
\hline $\mathrm{M}_{1}$ & $13.76 / 0.25$ & 0.465 & 488 & 0.714 & 0.714 \\
\hline $\mathrm{M}_{2}$ & $13.76 / 0.25$ & 0.465 & 488 & 0.714 & 0.714 \\
\hline $\mathrm{M}_{3}$ & $13.76 / 0.25$ & 0.465 & 488 & 0.714 & 0.714 \\
\hline $\mathrm{M}_{4}$ & $10.64 / 0.25$ & 0.449 & 488 & 1.086 & 1.086 \\
\hline $\mathrm{M}_{5}$ & $21.28 / 0.25$ & 0.449 & 976 & 1.086 & 1.086 \\
\hline
\end{tabular}

Table 6.1. The transistor parameters of the simulated delay cell 
Chapter 6. Compact Cascadable gm-C All-Pass True Time Delay Cell with Reduced Delay Variation over Frequency

$\mathrm{f}_{\varphi=0}$ is evaluated at operation frequency of $1.75 \mathrm{GHz}$ (in the middle of the band $1-2.5 \mathrm{GHz}$ ). $\mathrm{f}_{\varphi=0}$ improves from $-0.52 \mathrm{GHz}$ for $\mathrm{R}=0 \Omega$ to $0.06 \mathrm{GHz}$ for $\mathrm{R}=1.5 \mathrm{k} \Omega$ (optimum). In terms of delay variation over 1 $2.5 \mathrm{GHz}$, using (6.2), a delay variation reduction from $9.8 \%$ for $\mathrm{R}=0$, to $1.4 \%$ for $\mathrm{R}=1.5 \mathrm{k} \Omega$ is found. The phase linearization resistor increases the noise figure of the delay cell by about $1.7 \mathrm{~dB}$.

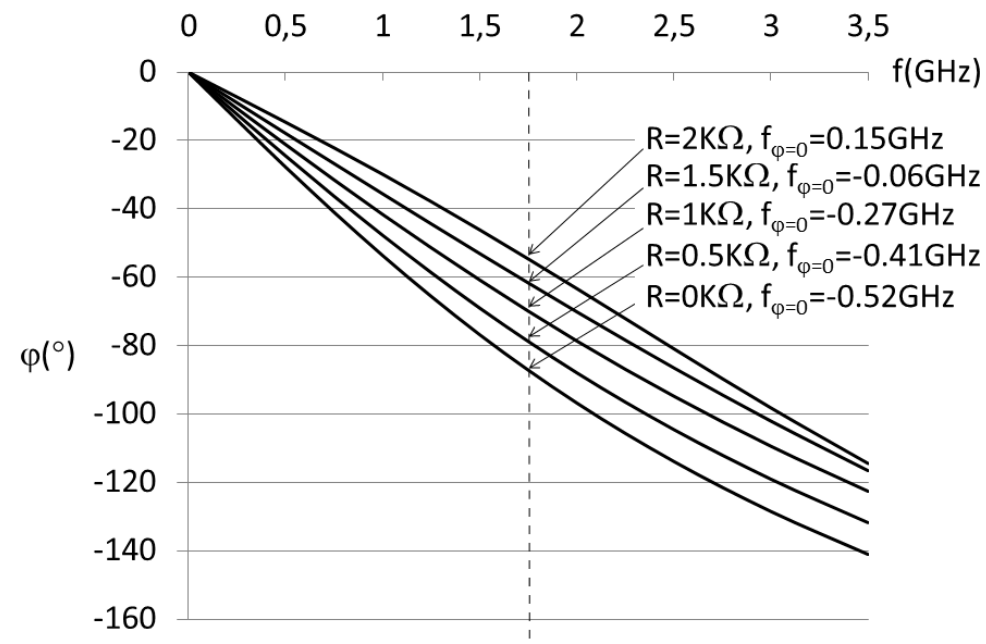

Fig. 6.10. Simulation results of the phase linearization technique for the parameters shown in Table 6.1

B. Bandwidth extension

The load capacitor plus the parasitic capacitors at the output of the delay cell $\left(\approx \mathrm{C}_{\mathrm{L}}+\mathrm{C}_{\mathrm{gs} 3}\right)$ cause an unwanted pole and consequently gain roll-off plus an extra amount of delay. In a cascade of identical delay cells, the total load plus parasitic capacitance at the output node is $3 \mathrm{C}_{\mathrm{gs}, \mathrm{M} 1}$. Therefore, the parasitic pole at output is: $\mathrm{f}_{\mathrm{p}, \mathrm{out}} \approx \mathrm{g}_{\mathrm{m} 3} /\left(6 \pi \mathrm{C}_{\mathrm{gs}, \mathrm{M} 1}\right)$. 
Chapter 6. Compact Cascadable gm-C All-Pass True Time Delay Cell with Reduced Delay Variation over Frequency

An active inductive peaking technique [18] is used for bandwidth extension by adding resistor $\mathrm{R}_{\mathrm{BWE}}$ to convert the diode connected transistor $\mathrm{M}_{3}$ to an "active inductor" (Fig. 6.11). The impedance of the active inductor (the part inside the dotted box) is:

$Z_{A-i n d}(s)=\frac{1}{g_{m 3}} \frac{s R_{B W E} \cdot C_{g s, m 3}+1}{s \frac{C_{g s, M 3}}{g_{m 3}}+1}$

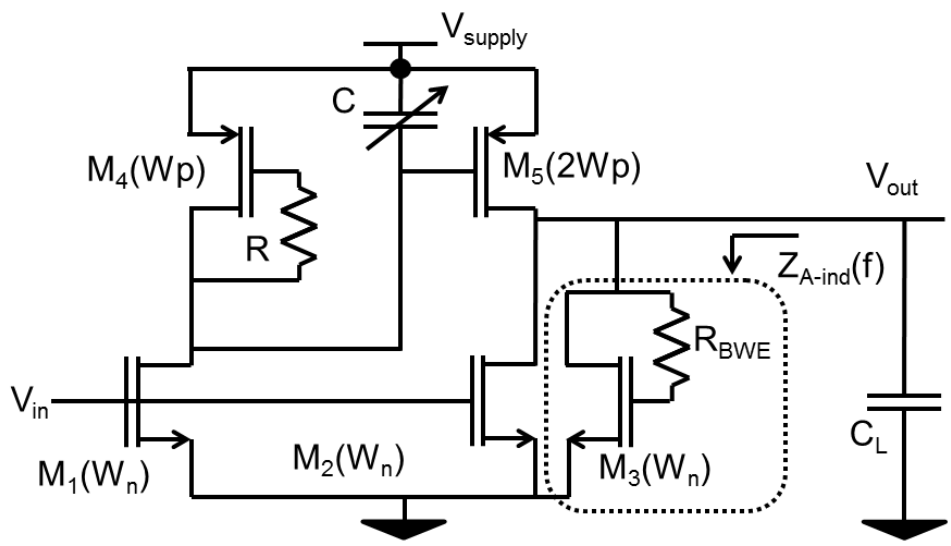

Fig. 6.11. Bandwidth extension of the filter

Choosing $\mathrm{R}_{\mathrm{BWE}}=1 / \mathrm{g}_{\mathrm{m} 3}$ results in $\mathrm{Z}_{\mathrm{A} \text {-ind }}=1 / \mathrm{g}_{\mathrm{m} 3}$. Therefore, the pole at the output becomes $\mathrm{f}_{\mathrm{BWE}}=\mathrm{g}_{\mathrm{m} 3} /\left(4 \pi \mathrm{C}_{\mathrm{gs}, \mathrm{M} 1}\right)$ which means $50 \%$ bandwidth extension. Fig. 6.12 shows the gain curve with $\mathrm{R}_{\mathrm{BWE}}$ as a parameter. The transistor parameters are the same as in table 1 . Theoretically, $50 \%$ bandwidth extension happens at $R_{B W E}\left(=1 / g_{m, M 3}\right)=298 \Omega$, however, because of extra parasitic capacitance due to $\mathrm{C}_{\mathrm{db}, \mathrm{M} 2}, \mathrm{C}_{\mathrm{db}, \mathrm{M} 5}, \mathrm{C}_{\mathrm{db}, \mathrm{M} 3}$, $\mathrm{C}_{\mathrm{gd}, \mathrm{M} 2}, \mathrm{C}_{\mathrm{gd}, \mathrm{M} 3}$ and $\mathrm{C}_{\mathrm{gd}, \mathrm{M} 5}$ and also finite output impedances of $\mathrm{M}_{3}, \mathrm{M}_{2}$ and $\mathrm{M}_{5}$, simulation shows a $33 \%$ bandwidth extension. The DC gain drop of $-2 \mathrm{~dB}$ is caused by the finite output impedance of the transistors. The bandwidth extension resistor $\left(\mathrm{R}_{\mathrm{BWE}}\right)$ increases the noise figure of the delay cell by about $0.6 \mathrm{~dB}$. In the following subsections a technique is introduced to compensate the DC gain drop. 
Chapter 6. Compact Cascadable gm-C All-Pass True Time Delay Cell with Reduced Delay Variation over Frequency

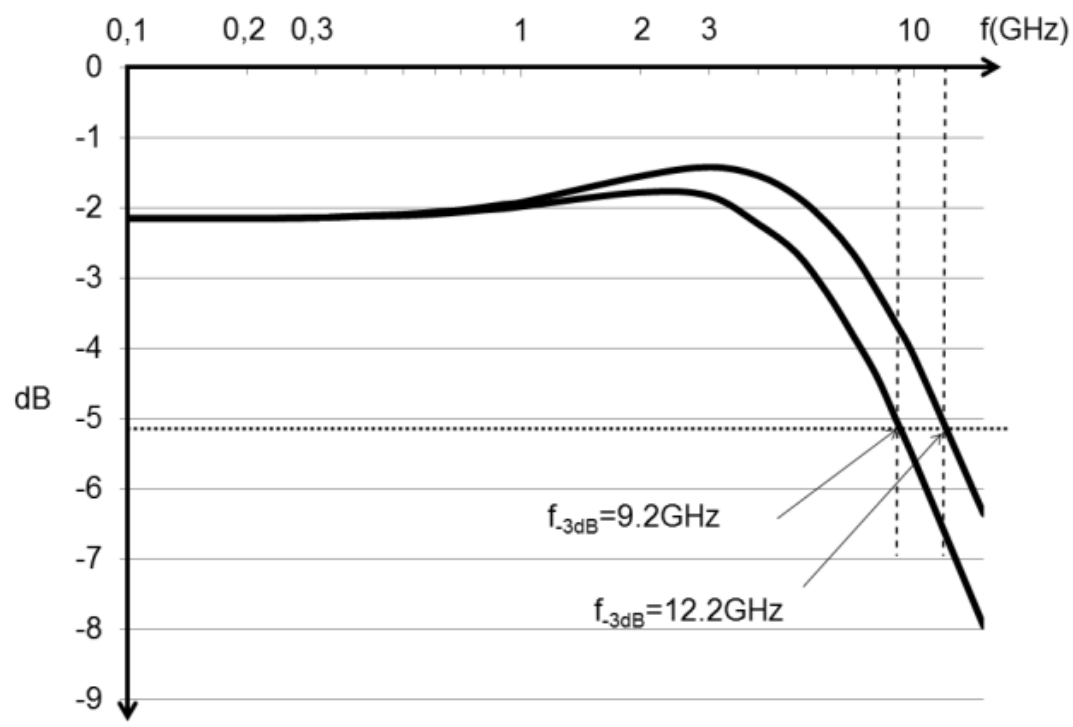

Fig. 6.12. Simulation of bandwidth extension technique $\left(\mathrm{R}_{\mathrm{WBE}}\right.$ as a parameter)

C. Binary tuning of the delay

Referring to equation (6.5), delay can be fine-tuned by varying $C$, which is designed as a 3 bit switchable binary weighted capacitor bank (see Fig. 6.13). Because all capacitors of the bank are ACgrounded, referred to $\mathrm{V}_{\text {supply, }}$ they are easily switchable with PMOS transistors. 


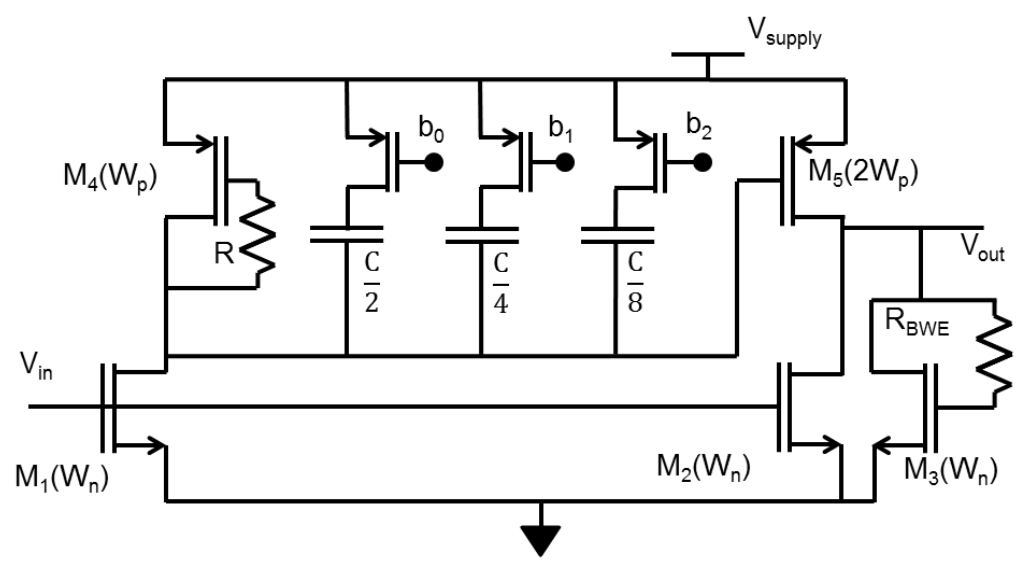

Fig. 6.13. The delay cell with 3 bit delay selection

D. Gain adjustment

The practically achieved DC-gain of the filter is less than one because of the finite output impedance of the transistors (refer to equation (6.6)). In a delay line gain errors add up and there may be a need to calibrate the gains to unity. For this purpose, a switchable structure consisting of $\mathrm{M}_{2, \mathrm{E}}$ and $\mathrm{M}_{3, \mathrm{E}}$ and $\mathrm{M}_{5, \mathrm{E}}$ has been added (Fig. 6.14). $M_{2, E}$ and $M_{5, E}$ work in parallel with $M_{2}$ and $M_{5}$ to increase their effective width by an amount equal to $\alpha \mathrm{W}$, so that the DC gain is multiplied by $1+\alpha$. Transistor $M_{3, E}$ sinks the excess DC current at the output point to keep the $\mathrm{DC}$ output bias voltage unchanged. $\mathrm{V}_{\mathrm{DC} \text {,bias }}$ is re-used from the biasing circuit (refer to Fig. 6.6). 
Chapter 6. Compact Cascadable gm-C All-Pass True Time Delay Cell with Reduced Delay Variation over Frequency

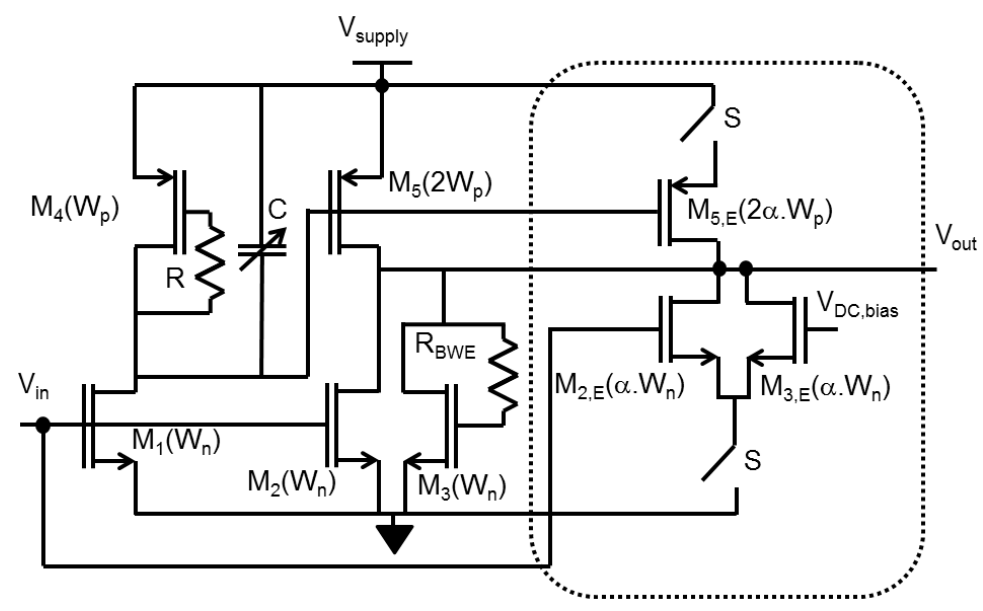

Fig. 6.14. Adding gain tuner to the delay cell

A set of binary weighted switchable gain tuning stages makes the tuning more precise (Fig. 6.15). 3 bits have been used for the DC-gain tuning in a gain-range of $3 \mathrm{~dB}(\mathrm{LSB} \approx 0.4 \mathrm{~dB})$.

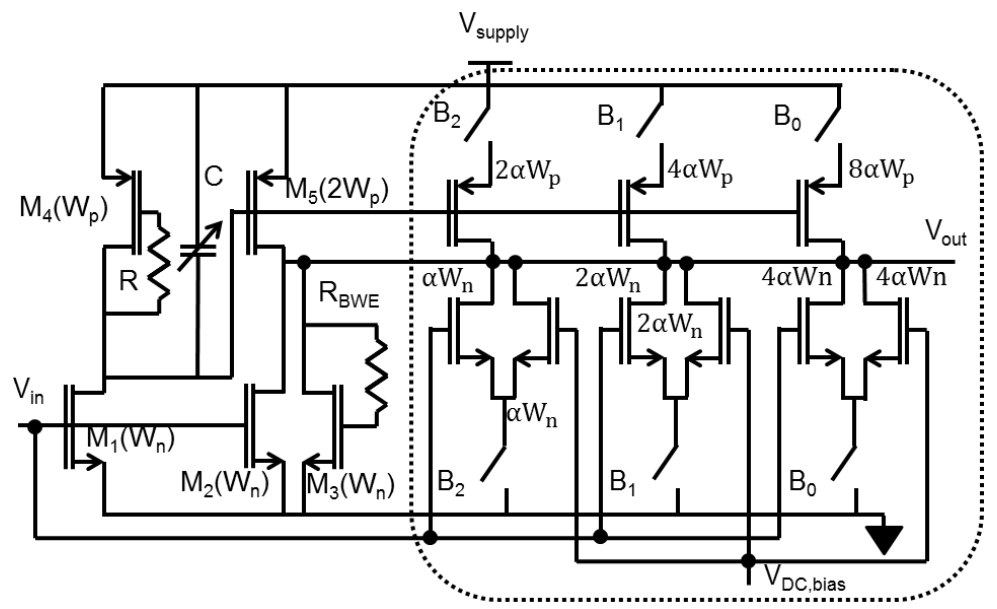

Fig. 6.15. Adding 3-bit gain calibration to the filter 
Chapter 6. Compact Cascadable gm-C All-Pass True Time Delay Cell with Reduced Delay Variation over Frequency

Table 6.2 shows a comparison between the simulated results of this work and the simulated results of other reported $\mathrm{g}_{\mathrm{m}} \mathrm{RC}$ delay cells (refer to Fig. 6.3). The technology used in every case is UMC $180 \mathrm{~nm}$ to compare to [13]. The $\mathrm{V}_{\mathrm{GS}}$ of NMOS transistors for all circuits are the same to maintain equal $\mathrm{f}_{\mathrm{t}, \mathrm{n}}=12.4 \mathrm{GHz}$ for fair comparison. As the table shows, the pole frequency of this work is much higher (more than 5x) than other works. The NSNR [15] (defined as SNR/P@IM3=1\%) criterion of the cells at $0.1 \mathrm{GHz}$ bandwidth was used to compare dynamic range. The NSNR of this work is $1 \mathrm{~dB}$ better than [11] and $6 \mathrm{~dB}$ less than [2], partly due to $\mathrm{IIP}_{3}$, but mainly due to the number of noise contributing devices of the new delay cell [12]. Clearly, the most strong point of this work is its frequency range which is much better than for other circuits in the same technology.

\begin{tabular}{|c|c|c|c|}
\hline & {$[11]$} & {$[2]$} & This work \\
\hline $\mathrm{V}_{\mathrm{GS}, \mathrm{nmos}}(\mathrm{V})$ & 0.714 & 0.714 & 0.714 \\
\hline $\mathrm{V}_{\mathrm{GS}, \mathrm{pmos}}(\mathrm{V})$ & - & - & 1.086 \\
\hline $\mathrm{V}_{\text {th,nmos }}(\mathrm{V})$ & 0.427 & 0.427 & 0.427 \\
\hline $\mathrm{V}_{\text {th,pmos }}(\mathrm{V})$ & - & - & 0.449 \\
\hline $\mathrm{I}_{\mathrm{DC}}(\mathrm{mA})$ & 0.2 & 3.4 & 0.6 \\
\hline Pole $\mathrm{f}_{\mathrm{p}}(\mathrm{GHz})$ & 0.36 & 0.48 & 2.63 \\
\hline $\begin{array}{c}\text { Input referred noise } @ \mathrm{f}_{\mathrm{p}} \\
(\mathrm{nV} / \mathrm{sqrt}(\mathrm{Hz}))\end{array}$ & 12.8 & 2.4 & 6.2 \\
\hline $\mathrm{IIP}_{3}(\mathrm{dBm} @ 50 \Omega) @ \mathrm{f}_{\mathrm{p}}$ & 3 & 5 & 3 \\
\hline $\mathrm{V}_{\mathrm{IM} 3=1 \%}(\mathrm{mV})$ & 48 & 84 & 45 \\
\hline $\begin{array}{c}\text { SNR @ IM3=1\% in } \\
\text { BW=0.1GHz }\end{array}$ & 52 & 71 & 57 \\
\hline $\begin{array}{c}\text { Normalized SNR/P } \\
\left(1 \% \mathrm{IM}_{3}, 1 \mathrm{~Hz}, 1 \mathrm{~mW}[20]\right)\end{array}$ & 138 & 145 & 139 \\
\hline
\end{tabular}

Table 6.2. Comparison between simulated delay cells 


\subsection{Beamforming System Design}

In this section the timed array system specifications are related to the delay cell requirements. The formulas of this section are used in section 6.7 to find the specifications of the sub-blocks in timed array antenna systems. Suppose we aim at $\mathrm{N}$ antenna elements, a frequency band from $f_{\min }$ to $f_{\max }$, a maximum steering angle $\pm \theta_{\max }$ w.r.t. the boresight and $b$ bits of spatial steering resolution, while the required noise figure is less than $\mathrm{NF}_{\text {max. }}$. No grating lobes should exist and $<-40 \mathrm{~dB}$ null depth is targeted. From these specifications, system design parameters are extractable using [3] and [4], like the distance between antenna elements, maximum required delay, number of delay steps, and the noise figure of each channel.

To avoid grating lobes, the distance between antenna elements (d) must be less than half the wave length at the maximum operating frequency $\left(f_{\max }\right)$

$d \leq \frac{\lambda_{f \max }}{2}$

The noise figure for $\mathrm{N}$ antennas improves with $10 \log (\mathrm{N})$ [dB] w.r.t. the noise figure for a single antenna channel. The maximum required delay per channel $\left(\tau_{\max }\right)$ depends on: 1$)$ the number of antenna elements $(N), 2)$ the distance between antenna elements (d), 3) the maximum steering angle $\left(\theta_{\max }\right)$. It can be expressed as [4] ( $\mathrm{c}$ is the speed of the waves in the space):

$$
\tau_{\max }=(N-1) \frac{\text { d.sin }\left(\theta_{\max }\right)}{c}
$$

The minimum delay step ( $\left.\tau_{\min }\right)$ depends on: 1 ) distance between antenna elements $(d), 2)$ maximum steering angle $\left(\theta_{\max }\right)$ and 3 ) spatial resolution in bits (b)

$$
\tau_{\min }=\frac{d \cdot \cos \left(\theta_{\max }\right)}{C} \cdot \frac{\theta_{\max }}{2^{b-1}}
$$


Chapter 6. Compact Cascadable gm-C All-Pass True Time Delay Cell with Reduced Delay Variation over Frequency

The null depths are ideally equal to $-\infty$, but gain mismatch will decrease the null depths. Timed array system simulations show that for a 4-antenna array, and null depths less than $-40 \mathrm{~dB}$, less than $0.06 \mathrm{~dB}$ gain difference between the channels is required.

\subsection{4 channel wideband beamforming IC}

The designed delay cell is the basic building block of the time delay based timed array antenna IC. The IC has four antenna channels (Fig. 6.16) [12].

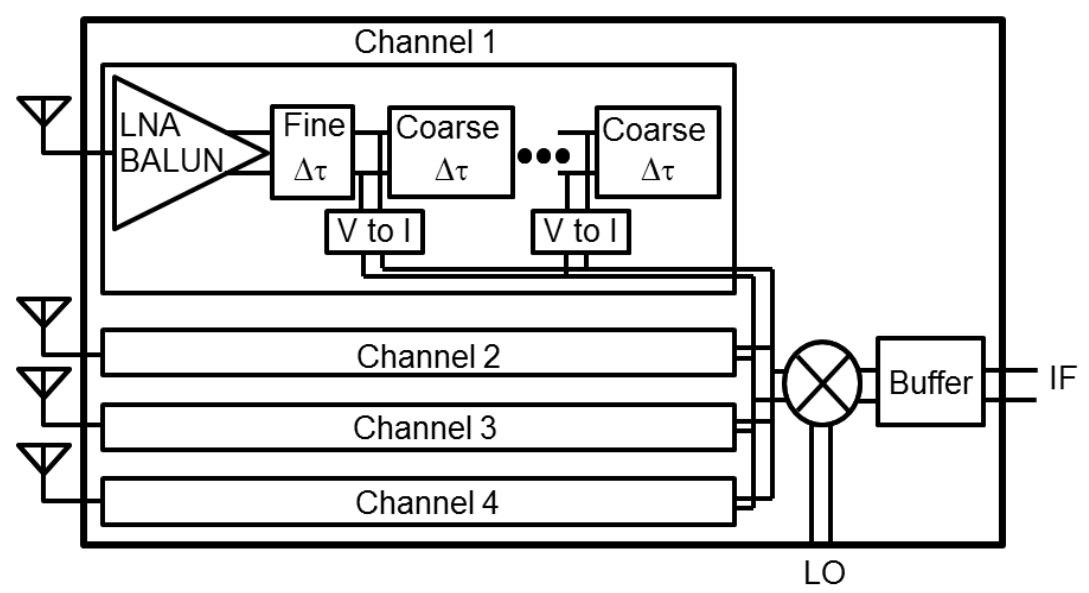

Fig. 6.16. The timed array IC: block level [12]

Each channel applies adjustable delay and gain on the input signal. As shown in Fig. 6.16, the adjustable delay is a combination of "Fine $\Delta \tau$ " cells cascaded with "Coarse $\Delta \tau$ " delay cells. The "Fine $\Delta \tau$ " is realized by a cascade of three delay cells with small delay steps (refer to Fig. 6.13). Each "Coarse $\Delta \tau$ " is a cell with large delay steps (refer to Fig. 6.15). In section 6.7 it will be shown that 550ps total delay has been realized in a 5 bit delay resolution via "Fine $\Delta \tau$ " and 6 cascaded "Coarse $\Delta \tau$ " cells. The last "Coarse $\Delta \tau$ " cell acts as a termination. An LNA/BALUN precedes the delay chain to reduce the noise figure. The output signals 
Chapter 6. Compact Cascadable gm-C All-Pass True Time Delay Cell with Reduced Delay Variation over Frequency

of the lines are added to each other to complete the beam forming function. Then the signal is down-converted to IF via a mixer and external LO. The total noise of the chip depends on the beam direction because the amount of the delay at each channel (number of cascaded coarse $\Delta \tau$ cells) changes with the beam direction. The maximum noise figure occurs when the beam directs toward the maximum steering angle $\theta_{\text {max. }}$ In this case the average delay of the channels is at maximum.

Analysis based on a Taylor series expansion shows that distortion has only minor impact on the phase of the fundamental frequency. Therefore the position of the null and its depth doesn't change much. However, strong signals may also generate higher harmonics with different phases than the fundamental signal in each antenna channel. After summation, the amplitude of the harmonics can add up and cause high frequency interference even if a signal comes from a null direction. Whether this is a problem depends on specific requirements and boundary conditions which are outside the scope of this paper. Below, the functionality and circuit structure of the sub-blocks are explained.

\section{A. LNA/BALUN}

The LNA/BALUN has four main functions: 1) antenna impedance matching, 2) low noise amplification, 3) Single to differential conversion (BALUN) and 4) Gain tuning. The single to differential conversion makes the signals less sensitive to interference from other channels. It exploits a noise cancelling common gate (CG)-common source (CS) structure (Fig. 6.17) [19]. The DC blocking capacitors $C_{c g}$ $\mathrm{C}_{\mathrm{cs}}$ and $\mathrm{C}_{\mathrm{csg}}$ are the only series capacitors in each channel. Due to a design error, their parasitic capacitance to the substrate is the main cause of the bandwidth limitation. $\mathrm{V}_{\text {out,cg }}$ and $\mathrm{V}_{\text {out,cs }}$ are DC fed to the "Fine $\Delta \tau$ " block. The AC gain in CG, CS stages of the LNA/BALUN can be trimmed by controlling bias voltages $V_{b 1}$ and $V_{b 2}$ to provide gain equalization and calibration. A 4 bit DAC is used to cover $1 \mathrm{~dB}$ gain variation with $0.06 \mathrm{~dB}$ as LSB step. This small range hardly degrades $\mathrm{S}_{11}$ (it remains less than $-10 \mathrm{~dB}$ ) but provides the required gain resolution to provide $<-40 \mathrm{~dB}$ null depths. 


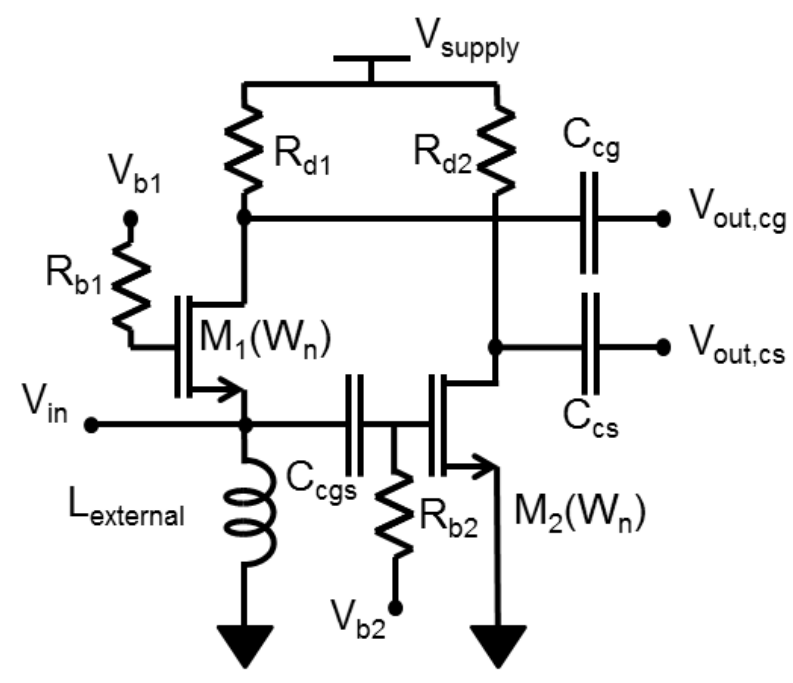

Fig. 6.17. LNA/BALUN

B. Fine delay control

The "fine $\Delta \tau$ " block realizes small delay steps. It consists of 3 cascaded delay adjustable cells (Fig. 6.13), to cover one coarse delay step with extra margin for PVT, to prevent "missing bits". The gain of the fine $\Delta \tau$ blocks are the same for all antenna channels, therefore, it doesn't affect the beam pattern and consequently they do not require gain calibration.

\section{Coarse delay control}

The Coarse $\Delta \tau$ delay line consists of six cascaded delay cells, each with a fixed delay and an adjustable amount of gain. At each (voltage) output of a coarse delay cell there is a V to I converter (see Fig. 6.16) which can be activated or not. This acts as a selectable tap to effectively change the length (and the delay amount) of the delay line. The gain adjustability of the stages is to calibrate the gain of the coarse delay line to unity, independent of the number of cascaded blocks. 
Chapter 6. Compact Cascadable gm-C All-Pass True Time Delay Cell with Reduced Delay Variation over Frequency

\section{Selectable V to I converters}

The selectable $\mathrm{V}$ to I converters fulfill two tasks: They select the desired output of the delay chain and they convert the signal from voltage to current. The input capacitance of the $\mathrm{V}$ to I converter has an effect on the delay of the channel but because this delay shift is equal for all channels it does not affect the beam pattern. However, they limit the bandwidth. Because the signals are converted to current, the summation function required for beam forming can be implemented by simply connecting all outputs together.

\section{E. LO, Mixer and output buffer}

An external differential LO is used to down-convert the beam formed signal to IF. The circuit and its outputs are differential and an active gilbert cell mixer is used with load resistors. The output voltage is buffered via source followers to match the output impedance to $50 \Omega$.

\subsection{Chip Implementation and Measurements}

To demonstrate wideband beamforming, a 4-channel beam forming chip was designed in 160nm CMOS, covering more than one octave of bandwidth from $1 \mathrm{GHz}$ to $2.5 \mathrm{GHz}$. The beam can be steered from $-60^{\circ}$ to $60^{\circ}$ related to bore-sight in 4 bits resolution. To avoid grating lobe conditions, the required inter-element antenna distance is $0.5 \lambda_{2.5 \mathrm{GHz}} \approx 6 \mathrm{~cm}$ (refer to (6.15)). The maximum required delay in each channel is found from (6.16) and is: $\tau_{\max }=510 \mathrm{psec}$. The delay step size is derived from the 4 bit beam steering resolution (refer to (6.17)): $\tau_{\min } \approx 13 p s e c$. The $\tau_{\max }$ to $\tau_{\min }$ ratio shows that for 4 bit steering angle resolution, 5 bit delay-resolution is needed per channel. The targeted average noise figure of the channels when the beam steers towards $\theta_{\max }= \pm 60^{\circ}$ is $8 \mathrm{~dB}$ at the mid of the frequency range $\left(\mathrm{f}_{0}=1.75 \mathrm{GHz}\right)$, i.e. the noise figure of each channel at $255 \mathrm{psec}$ delay must be $8 \mathrm{~dB}$. The $255 \mathrm{psec}$ delay consists of 3 cascaded coarse $\Delta \tau$ cells besides fine $\Delta \tau$. A single ended to differential voltage gain of $13 \mathrm{~dB}$ for each channel and 3.5dB noise figure for the LNA/BALUN theoretically results in $8.9 \mathrm{~dB}$ noise figure for every individual delay cell. Keeping 
Chapter 6. Compact Cascadable gm-C All-Pass True Time Delay Cell with Reduced Delay Variation over Frequency

overdrive voltage of transistors similar to table 1 results in $3.6 \mathrm{~mA}$ current for each individual delay cell. In this test chip the simple bias circuit of Fig 6.6 consisting of a diode connected N- and PMOS was used. Reduction of the supply directly decreases the current and consequently affects the $g_{m}$, noise and time-delay of the delay cell. To stabilize performance either a voltage regulator will be needed, or a bias current source should be used to bias $M_{6}$ and $M_{7}$ in Fig. 6.6. Fig. 6.18 shows the chip photograph.

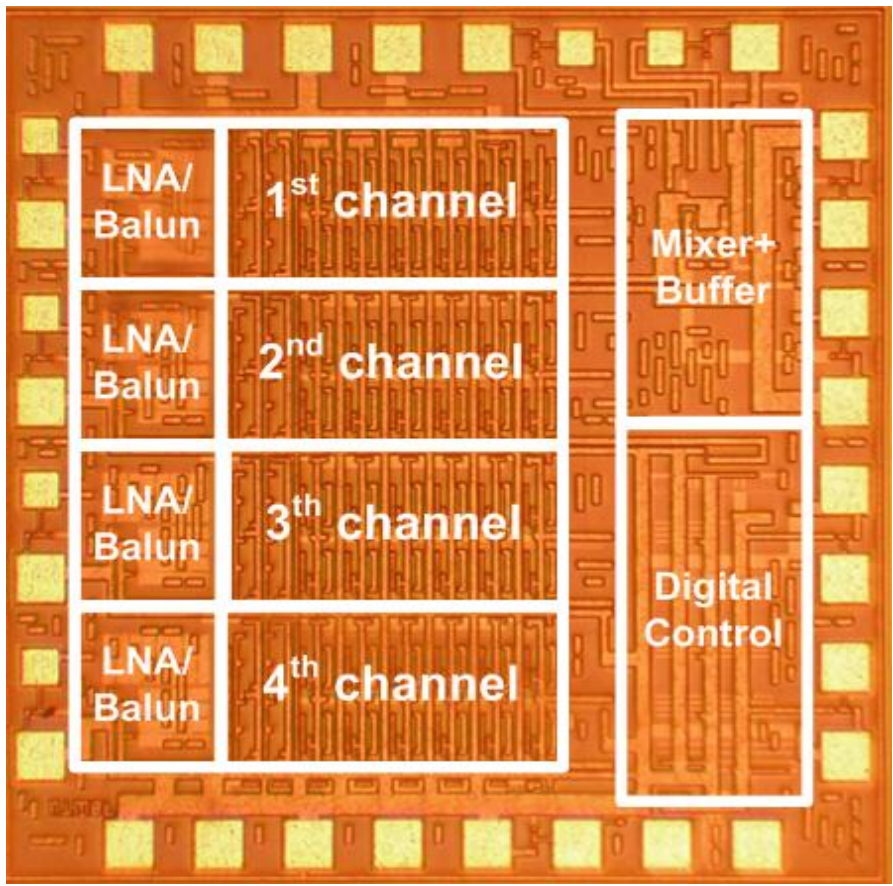

Fig. 6.18. The chip photograph

For each channel, the delay vs. frequency over the whole frequency band and for all settings was measured. An effective number of bits for the delay setting equal to $\mathrm{ENOB}=4.7(\mathrm{NOB}=5)$ was found (Fig. 6.19). The delays are approximately constant within $<10$ psec variation in the operating frequency band of $1-2.5 \mathrm{GHz}$. The flatness of the delay 
Chapter 6. Compact Cascadable gm-C All-Pass True Time Delay Cell with Reduced Delay Variation over Frequency

curves in Fig. 6.19 is an immediate result of applying the technique in Fig. 6.9 and Fig. 6.10 to linearize the phase vs. frequency and demonstrates that the optimization approach is highly effective. Substituting the maximum delay variations (10psec) and the maximum amount of delay $\left(\tau\left(\mathrm{f}_{0}\right)=550 \mathrm{psec}\right)$ in $(6.2)$, we find $\mathrm{f}_{\varphi=0}$ $\approx 0.06 \mathrm{GHz}$ at the mid of the frequency band $\left(\mathrm{f}_{0}=1.75 \mathrm{GHz}\right)$ which is close to the circuit simulations shown in Fig. 6.10

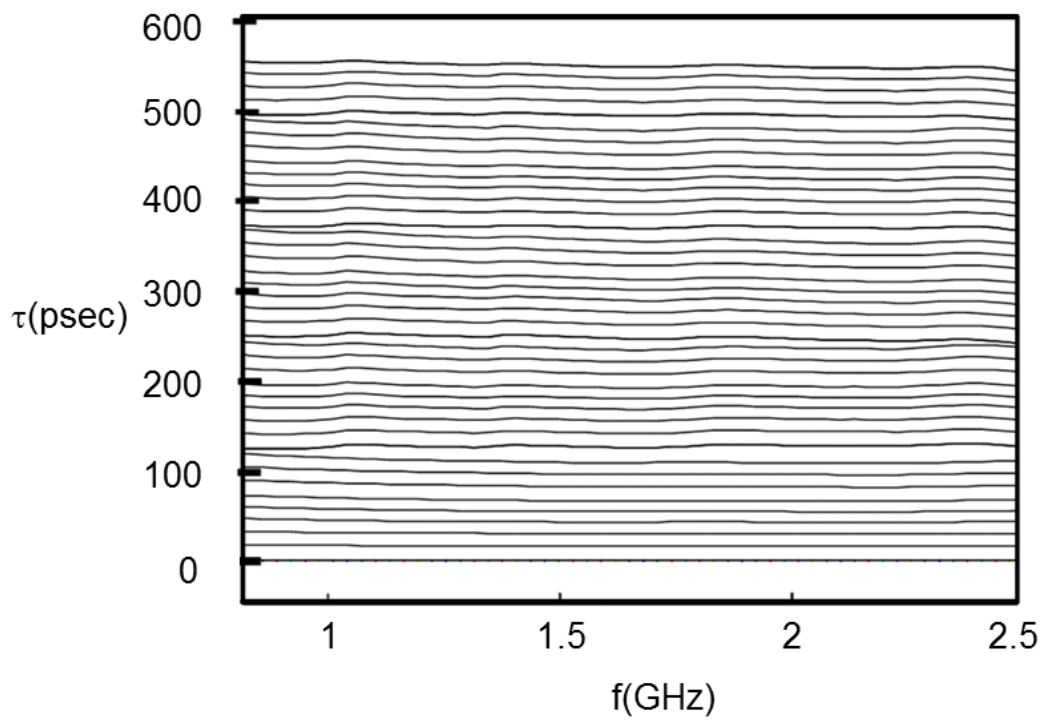

Fig. 6.19. Delay versus frequency for all delay settings.

Fig. 6.20 shows the gain vs. frequency variations for all delay and gain settings (without the effect of the LNA/BALUN gain trimming). For all delay settings the gain varies less than $\pm 1.8 \mathrm{~dB}$ at each individual frequency point from $1 \mathrm{GHz}$ to $2.5 \mathrm{GHz}$ band. For each delay setting (A fixed delay) the gain vs. frequency variations from $1 \mathrm{GHz}$ to $2.5 \mathrm{GHz}$ remains less than $2.8 \mathrm{~dB}$ (or $\pm 1.4 \mathrm{~dB}$ with respect to its average). The gain adjustability in the BALUN provides another opportunity to trim the gain of the individual frequency points with $0.03 \mathrm{~dB}$ resolution. The gain variations vs. all delay amount settings with the help of BALUN gain trimming is: $\pm 0.03 \mathrm{~dB}$ over 1 to $2.5 \mathrm{GHz}$ band (non-simultaneous) 
Chapter 6. Compact Cascadable gm-C All-Pass True Time Delay Cell with Reduced Delay Variation over Frequency

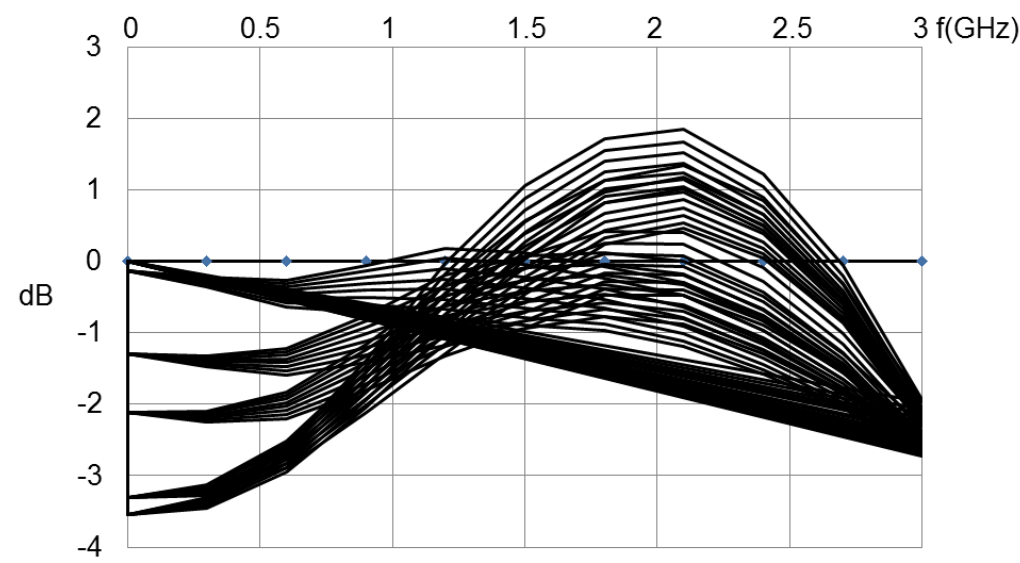

Fig. 6.20. Gain of the delay line (fine tune and coarse tune) vs. $f$ for all delay settings

This gain equality resolution results in deep null depths of the beam pattern which will be shown later (Fig. 6.22). The gain, noise figure and input matching $\left(\mathrm{S}_{11}\right)$ vs. frequency of a single receiver channel set at $255 \mathrm{psec}$ delay is shown in Fig. 6.21. The 255psec is the average delay of the 4 channels when the beam steers towards its maximum angle $\left(\theta_{\max }\right)$ which results in the maximum noise figure for the timed array (worst case scenario). For other steering angles the average delay of the channels is less and therefore the noise figure is better. The measured gain and noise figure is in agreement with the simulations within $0.9 \mathrm{~dB}$. 
Chapter 6. Compact Cascadable gm-C All-Pass True Time Delay Cell with Reduced Delay Variation over Frequency

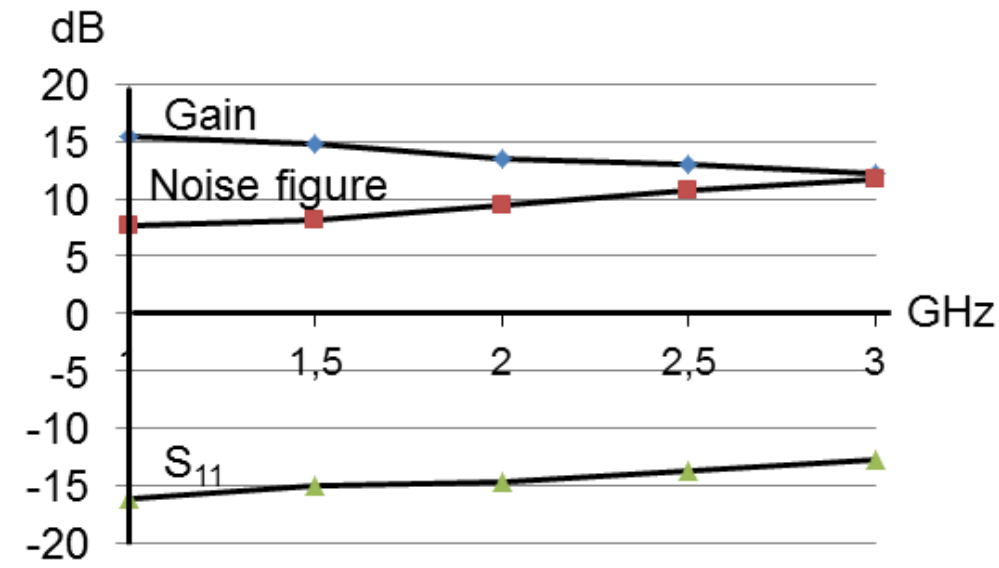

Fig. 6.21. Gain, input matching and noise figure when delay of the channel is 255 ps.

The measured beam pattern was compared with a simulated ideal time delay based beam forming system as shown in Fig. 6.22. For the frequency range from 2 to $2.5 \mathrm{GHz}$, the $-3 \mathrm{~dB}$ beam width varies from $63^{\circ}$ to $51^{\circ}$ and the null to null distance from $37^{\circ}$ to $29^{\circ}$. Also a new null appears at $-38^{\circ}$ in the pattern both in measurement and simulation.

The method used for beam pattern measurement is as follows: $4 \mathrm{RF}$ signals representing the antenna signals are generated via 4 external RF generators. Experiments were done at $2 \mathrm{GHz}$ and $2.5 \mathrm{GHz}$, while an external $3 \mathrm{GHz}$ signal is applied to the LO input. The beam formed signal is down-converted to $500 \mathrm{MHz}$ to $1 \mathrm{GHz}$. Going through all delay settings the beam patterns for $2 \mathrm{GHz}$ and $2.5 \mathrm{GHz}$ are synthesized. Comparing to the simulated beam pattern, it can be seen (Fig. 6.22) that spatial directions for the beam and nulls in the measured pattern closely follows the ideal pattern. The null depths of the beam pattern was limited to $-24 \mathrm{~dB}$ which is caused by the cross talk between the off-chip transmission lines of the antenna channels. 
Chapter 6. Compact Cascadable gm-C All-Pass True Time Delay Cell with Reduced Delay Variation over Frequency

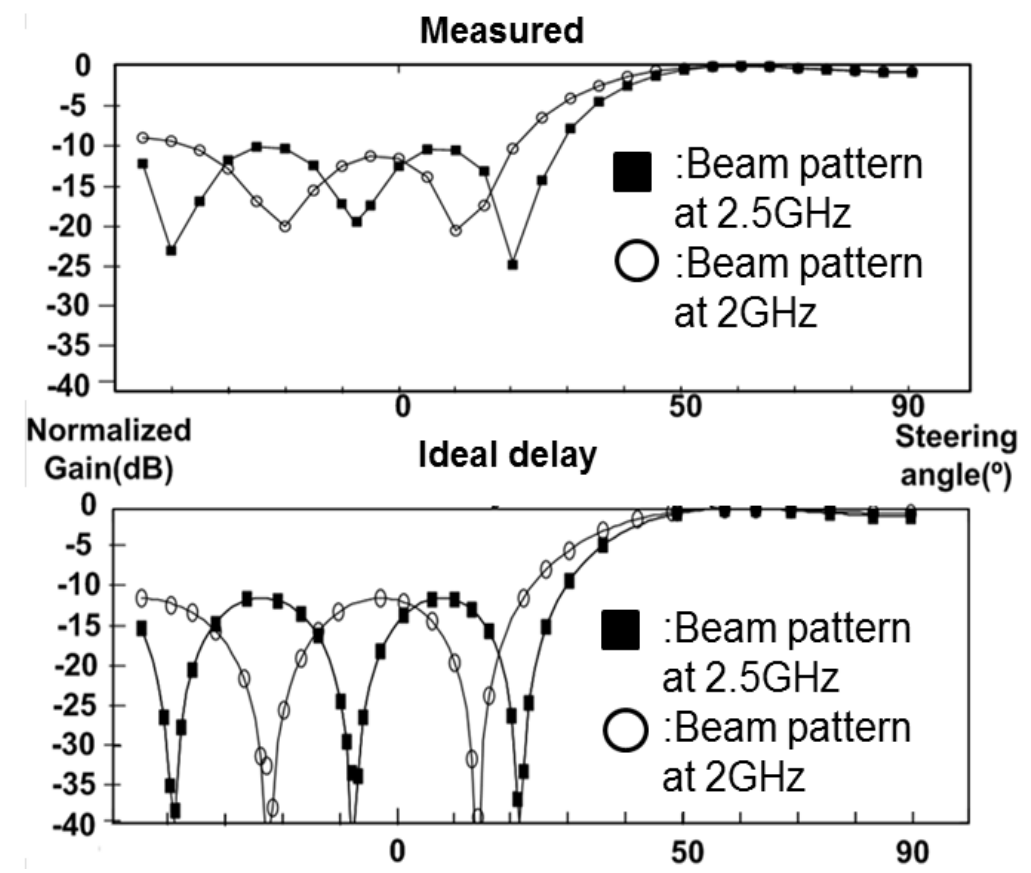

Fig. 6.22. The measured beam pattern compared to a simulated ideal beam pattern.

Table 6.3 [24] compares several reported delay circuits implemented via different technologies and topologies. Compared to other methods, the proposed circuit provides the lowest amount of delay vs. frequency variation (1.8\% over more than an octave of bandwidth). Also it is the most compact delay circuit which provides between 1 and 2 orders of magnitude more delay per area. Therefore this circuit is one of the best candidates for low $\mathrm{GHz} \mathrm{RF}$ band applications requiring large amounts of delay. 
Chapter 6. Compact Cascadable gm-C All-Pass True Time Delay Cell with Reduced Delay Variation over Frequency

\begin{tabular}{|c|c|c|c|c|c|}
\hline & [21] & [22] & [23] & [9] & [24] \\
\hline Technology & $\begin{array}{c}0.18 \mu \mathrm{m} \\
\mathrm{SiGe}\end{array}$ & $\begin{array}{l}0.13 \mu \mathrm{m} \\
\mathrm{SiGe}\end{array}$ & $\begin{array}{c}0.13 \mu \mathrm{m} \\
\mathrm{SiGe}\end{array}$ & $\begin{array}{c}0.13 \\
\mu \mathrm{m} \\
\mathrm{CMOS}\end{array}$ & $\begin{array}{l}0.8 \mu \mathrm{m} \\
\mathrm{SiGe}\end{array}$ \\
\hline $\begin{array}{c}\text { Supply } \\
\text { Voltage(V) }\end{array}$ & 2.5 & 2.5 & 2.5 & 1.5 & 2.5 \\
\hline $\begin{array}{l}\text { Gain } \\
\text { variation } \\
(\mathrm{dB})\end{array}$ & \pm 1 & N/A & N/A & \pm 3 & \pm 0.7 \\
\hline $\begin{array}{c}\text { Max. } \\
\text { delay(ps) }\end{array}$ & 64 & 16 & 54 & 225 & 25 \\
\hline $\begin{array}{c}\text { Frequency } \\
{[\mathrm{GHz}]}\end{array}$ & $1-15$ & $55-65$ & $31-41$ & $1-15$ & $3-10$ \\
\hline $\begin{array}{c}\text { Delay } \\
\text { variation }\end{array}$ & $\sim 16 \%$ & N/A & N/A & $\sim 14 \%$ & $40 \%$ \\
\hline $\begin{array}{c}\mathrm{P}_{\mathrm{DC}} / \text { channel } \\
(\mathrm{mW})\end{array}$ & 87.5 & - & 104 & 78 & 38.5 \\
\hline $\begin{array}{l}\text { Resolution } \\
\text { (ps) }\end{array}$ & 4 & 1.2 & 18 & 15 & Cont. \\
\hline $\operatorname{Size}\left(\mathrm{mm}^{2}\right)$ & 0.82 & 0.35 & 1.44 & 1.5 & 0.23 \\
\hline $\begin{array}{c}\text { Delay/Size } \\
\left(\mathrm{ps} / \mathrm{mm}^{2}\right)\end{array}$ & 78 & 45 & 37.5 & 150 & 109 \\
\hline
\end{tabular}

(1 $1^{\text {st }}$ part)

Table 6.3.a. Benchmarking and comparison between different reported delay cells (rest of the table is in the next page). 
Chapter 6. Compact Cascadable gm-C All-Pass True Time Delay Cell with Reduced Delay Variation over Frequency

\begin{tabular}{|c|c|c|c|c|c|c|}
\hline & [25] & \multicolumn{2}{|c|}{ [26] } & \multicolumn{2}{|c|}{ [26] } & $\begin{array}{l}\text { This } \\
\text { work }\end{array}$ \\
\hline Technology & $\begin{array}{c}0.09 \\
\mu \mathrm{m} \\
\mathrm{CMOS}\end{array}$ & \multicolumn{2}{|c|}{$\begin{array}{c}0.25 \\
\mu \mathrm{m} \\
\mathrm{SiGe}\end{array}$} & \multicolumn{2}{|c|}{$\begin{array}{c}0.25 \\
\mu \mathrm{m} \\
\mathrm{SiGe}\end{array}$} & $\begin{array}{c}0.14 \\
\mu \mathrm{m} \\
\mathrm{CMOS}\end{array}$ \\
\hline $\begin{array}{c}\text { Supply } \\
\text { Voltage(V) }\end{array}$ & N/A & \multicolumn{2}{|c|}{2.7} & \multicolumn{2}{|c|}{1.5} & 1.5 \\
\hline $\begin{array}{c}\text { Gain } \\
\text { variation }(\mathrm{dB})\end{array}$ & \pm 3 & \multicolumn{2}{|c|}{ \pm 0.9} & \multicolumn{2}{|c|}{ \pm 1.4} & \pm 1.4 \\
\hline Max. delay(ps) & 26 & \multicolumn{2}{|c|}{12} & \multicolumn{2}{|c|}{12.5} & 550 \\
\hline $\begin{array}{c}\text { Frequency } \\
{[\mathrm{GHz}]}\end{array}$ & $0-8$ & $20-40$ & $25-35$ & $20-40$ & $25-35$ & $1-2.5$ \\
\hline Delay variation & $10 \%$ & $6.4 \%$ & $3 \%$ & $6.6 \%$ & $3.2 \%$ & $1.8 \%$ \\
\hline $\begin{array}{c}\mathrm{P}_{\mathrm{DC}} / \text { channel } \\
(\mathrm{mW})\end{array}$ & - & \multicolumn{2}{|c|}{146} & \multicolumn{2}{|c|}{33} & 90 \\
\hline Resolution(ps) & 13 & \multicolumn{2}{|c|}{ Cont. } & \multicolumn{2}{|c|}{ Cont. } & 13 \\
\hline $\operatorname{Size}\left(\mathrm{mm}^{2}\right)$ & N/A & \multicolumn{2}{|c|}{0.1} & \multicolumn{2}{|c|}{0.1} & 0.07 \\
\hline $\begin{array}{c}\text { Delay/Size } \\
\left(\mathrm{ps} / \mathrm{mm}^{2}\right)\end{array}$ & N/A & \multicolumn{2}{|c|}{120} & \multicolumn{2}{|c|}{125} & 7857 \\
\hline
\end{tabular}

$\left(2^{\text {nd }}\right.$ part $)$

Table 6.3.b. Benchmarking and comparison between different reported delay cells (rest of the table is in the previous page).

Table 6.4 shows a comparison between our $\mathrm{g}_{\mathrm{m}}-\mathrm{RC}$ timed array chip and two other reported time delay based chips designed for beamforming, which exploit LC delay lines and transmission lines. The compactness of the delay cells allows us to implement the chip in much smaller area at comparable power consumption conditions. The reported noise figure of this circuit is higher, but it is for the worst case scenario (maximum steering angle: $\theta= \pm 60^{\circ}$ ). Steering to smaller angles (referred to the bore-sight) requires less delay and produces less noise. The reported amplitude vs. frequency variations $( \pm 1.4 \mathrm{~dB})$ is instantaneous for the $1-2.5 \mathrm{GHz}$ frequency band at each delay settings. The gain trimming property of the LNA plus gain calibration of the delay cells provide $0.03 \mathrm{~dB}$ resolution for individual frequencies. 
Chapter 6. Compact Cascadable gm-C All-Pass True Time Delay Cell with Reduced Delay Variation over Frequency

\begin{tabular}{|c|c|c|c|}
\hline & This work & $\begin{array}{c}\text { Chu, ISSCC } \\
2007 \text { [9] }\end{array}$ & $\begin{array}{c}\text { Van Vliet, } \\
\text { GAAS } 2003 \\
{[8]}\end{array}$ \\
\hline Technology & CMOS, $140 \mathrm{~nm}$ & CMOS, 130nm & $\begin{array}{l}\text { PHEMT, } \\
250 \mathrm{~nm}\end{array}$ \\
\hline Technique & $\mathrm{Gm}-\mathrm{C}$ & LC delay & $\begin{array}{l}\text { Transmission } \\
\text { line }\end{array}$ \\
\hline \multicolumn{4}{|c|}{ Features Per Antenna Channel } \\
\hline Gain & $\begin{array}{c}12-15 \mathrm{~dB} \\
\text { (f-dependent) }\end{array}$ & $10 \mathrm{~dB}$ & $6-9 \mathrm{~dB}$ \\
\hline Noise Figure & $8-10 \mathrm{~dB}$ & $2.9-4.8 \mathrm{~dB}$ & $4.3 \mathrm{~dB}$ \\
\hline IIP3 & $\begin{array}{l}-13 \text { to }-20 \mathrm{dBm} \\
\text { (min to max } \\
\text { delay length) }\end{array}$ & Not mentioned & Not mentioned \\
\hline $\begin{array}{l}-1 \mathrm{~dB} \text { compression } \\
\text { point }\end{array}$ & $\begin{array}{l}-21 \text { to }-28 \mathrm{dBm} \\
\text { (min to max } \\
\text { delay length) }\end{array}$ & Not mentioned & $5 \mathrm{dBm}$ \\
\hline $\begin{array}{l}\text { Amplitude variation } \\
\text { vs. f }\end{array}$ & $\pm 1.4 \mathrm{~dB}$ & $\pm 1 \mathrm{~dB}$ & $\pm 2.5 \mathrm{~dB}$ \\
\hline Delay resolution & $14 \mathrm{psec}$ & $15 \mathrm{psec}$ & $2.5 \mathrm{psec}$ \\
\hline Delay variation vs. f & $<10$ psec & $<40$ psec & $<20$ psec \\
\hline Maximum delay & $550 \mathrm{psec}$ & $225 \mathrm{psec}$ & 150psec \\
\hline $\begin{array}{c}\text { Current } \\
\text { consumption }\end{array}$ & $50 \mathrm{~mA}$ & $40 \mathrm{~mA}$ & Not mentioned \\
\hline \multicolumn{4}{|c|}{ Complete 4 channel beamformer } \\
\hline $\begin{array}{c}\text { Beam direction } \\
\text { resolution }\end{array}$ & $3.5 \mathrm{Bit}$ & $3.5 \mathrm{Bit}$ & $6 \mathrm{Bit}$ \\
\hline frequency range & $1-2.5 \mathrm{GHz}$ & $18 \mathrm{GHz}$ & $3-16 \mathrm{GHz}$ \\
\hline Power consumption & 250mA@1.8V & 370mA@1.5V & Not mentioned \\
\hline Die area & $1 \mathrm{~mm}^{2}$ & $10 \mathrm{~mm}^{2}$ & $10 \mathrm{~mm}^{2}$ \\
\hline
\end{tabular}

Table 6.4. Comparison between this works and two other time delay based phased array systems 


\subsection{Conclusions}

This chapter presented a compact all-pass $\mathrm{g}_{\mathrm{m}}-\mathrm{C}$ cell that was compared to other reported $\mathrm{g}_{\mathrm{m}}-\mathrm{RC}$ delay cells, showing $5 \mathrm{x}$ higher operating frequency range. A chip implementation of the delay cell in $160 \mathrm{~nm}$ CMOS results in a flat gain up to $2.5 \mathrm{GHz}$ for the delay cell, with the help of a bandwidth extension technique. The delay cells are directly cascadable to realize a delay line without AC-coupling or buffering. This avoids parasitic capacitances to ground from DC blocking capacitors which limit frequency range or require extra current consumption. The circuit exploits current re-use with a slow PMOS low-pass path in parallel to a fast NMOS unity gain path to maximize the useful frequency range. Bandwidth and phase linearity is further enhanced by adding carefully dimensioned resistors to the diode-connected transistors. Gain fine control in the delay cells allows for precise gain calibration, independent of delay. Using simulation, a direct comparison of the new delay cell with existing $\mathrm{g}_{\mathrm{m}}-\mathrm{C}$ and $\mathrm{g}_{\mathrm{m}}-\mathrm{RC}$ delay cells has been made in terms of frequency range, dynamic range and power consumption. The SNR/P at 1\% IM3 of the designed delay cell is $1 \mathrm{~dB}$ better than [11] and $6 \mathrm{~dB}$ worse than [2], while the frequency range is at least $5 x$ larger (compared to [2] and [11]). To validate performance, a 4 antenna beamforming receiver chip with a maximum steering angle $\theta_{\max }$ of $\pm 60^{\circ}$ was designed in $160 \mathrm{~nm}$ CMOS technology with a total delay per channel of 550 psec in an area of $0.15 \mathrm{~mm}^{2}$. Compared to other chips with LC delay lines and transmission lines, this is about 2 orders of magnitude more delay per area. The 550 psec delay is digitally controllable in 13psec steps. Delay variation over a bandwidth from 1 to $2.5 \mathrm{GHz}$ is less than 10 psec, which is only $1.8 \%$ of the realized delay. In other words, the selectable delays are monotonous, with low RMS error in the frequency band and therefore easy to use in calibration schemes. The delay/size of the circuit which is $7857 \mathrm{ps} / \mathrm{mm}^{2}$ is at least $50 \mathrm{x}$ more than other delay circuits reported in literature which makes it quite suitable for low $\mathrm{GHz}$ operations that need large amounts of delays. An effective delay resolution of $4.7 \mathrm{bits}$ is demonstrated which corresponds to an effective spatial beam steering resolution of 3.5 bits for full scale steering range of $\pm 60^{\circ}$, i.e. $10.6^{\circ}$ spatial angle resolution. The average noise figure of each antenna channel in the worst case 
Chapter 6. Compact Cascadable gm-C All-Pass True Time Delay Cell with Reduced Delay Variation over Frequency

scenario (when the average delay in 4 channels is maximum, i.e. a beam direction is at $\pm 60^{\circ}$ ) is $10 \mathrm{~dB}$.

\section{References}

[1] A. V. Oppenheim, R. W. Schafer and J. Buck, "Filter Design Techniques," in Discrete-Time Signal Processing, 2nd ed., New Jersey, Pretince-Hall, 1999, ch. 7, sec. 1, pp. 440-449.

[2] J. Buckwalter and A. Hajimiri, "An active analog delay and the delay reference loop," IEEE RFIC Symp., 2004, pp. 17-20.

[3] H. J. Visser, "Antennas," in Array and Phased Array Antenna Basics, West Sussex, England, Wiley, 2006, ch. 2, sec. 5, pp. 7680.

[4] R. J. Mailloux, "Phased Arrays in Radar and Communication Systems," in Phased Array Antenna Handbook, 2nd ed., Norwood, Artech house, 2005, ch.1, sec. 3, pp. 44-60.

[5] S. K. Garakoui, E. A. M. Klumperink, B. Nauta and F. E. van Vliet, "Phased-array antenna beam squinting related to frequency dependency of delay circuits," EuRad Conf., 2011, pp. 416-419.

[6] S. K. Garakoui, E. A. M. Klumperink, B. Nauta and F. E. van Vliet, "Time delay circuits: A quality criterion for delay variations versus frequency," IEEE ISCAS Proc., 2010, pp. 42814284.

[7] F. Ellinger, "Passive Devices and Networks," in Radio Frequency Integrated Circuits and Technologies, Berlin, Germany, Springer, 2007, ch. 6, sec. 1, pp. 195-198. 
Chapter 6. Compact Cascadable gm-C All-Pass True Time Delay Cell with Reduced Delay Variation over Frequency

[8] F. E. van Vliet, M. van Wanum, A. W. Roodnat and M. Alfredson, "Fully-integrated wideband TTD core chip with serial control," Gallium Arsenide applications symp., Munich, pp. 89-92, 2003.

[9] T. Chu, J. Roderick and H. Hashemi, "An Integrated UltraWideband Timed Array Receiver in $0.13 \mu \mathrm{m}$ CMOS Using a PathSharing True Time Delay Architecture," IEEE J.Solid-State Circuits, vol.42, no. 12, pp. 2834- 2850, Dec. 2007.

[10] B. Razavi, Design of Analog CMOS Integrated Circuits, New York: McGraw-Hill, 2001, ch. 12, sec. 2, pp. 410-423.

[11] P. Horowitz and w. Hill, "Unity-gainphase splitter," in The Art of Electronics, 2nd ed., New York, Cambridge University Press, 1999, ch. 2, sec. 8, pp. 77-78.

[12] S. K. Garakoui, E. A. M. Klumperink, B. Nauta and F. E. van Vliet, "A 1-to-2.5GHz phased-array IC based on gm-RC all-pass time-delay cells," IEEE ISSCC Dig. Tech. Papers, 2012, pp. 80-82.

[13] S. K. Garakoui, E. A. M. Klumperink, B. Nauta and F. E. van Vliet, "Frequency Limitations of First-Order gm - RC All-Pass Delay Circuits," IEEE T. on Circuits and Systems II, vol. 60, no. 9, pp. 572-576, Aug. 2013.

[14] K. Bult and H. Wallinga, "A CMOS analog continuous-time delay line with adaptive delay-time control," IEEE J. Solid-State Circuits, vol. 23, no. 3, pp. 759- 766, June 1988.

[15] K. Bult, "Harmonic Performance," in Analog Cmos square-law circuits, PhD Thesis, Enschede, University of Twente, 1988, ch. 6, sec. 4, pp. 93-98.

[16] B. Nauta, "Tuning," in Analog Cmos Filters for Very High Frequencies, Enschede, The Netherlands, Springer, 1992, ch. 5, 
Chapter 6. Compact Cascadable gm-C All-Pass True Time Delay Cell with Reduced Delay Variation over Frequency

sec. 2 , pp. $139-141$.

[17] B. Razavi, "Prospects of CMOS technology for high-speed optical communication circuits," Solid-State Circuits, IEEE Journal of , vol.37, no.9, pp. 1135-1145, Sep. 2002.

[18] T. H. Lee, "noise," in The Design of CMOS Radio-Frequency Integrated Circuits, 2nd ed., Cambridge University Press, 2003, ch. 11, pp. 361-362.

[19] S. C. Blaakmeer, E. A. M. Klumperink, D. M. W. Leenaerts and B. Nauta, "The BLIXER, a Wideband Balun-LNA-I/Q-Mixer topology," IEEE J. Solid-state Circuits, vol. 43, no.12, pp. 27062715, Dec. 2008.

[20] E. A. M. Klumperink and B. Nauta, "Systematic comparison of HF CMOS transconductors," IEEE T. on Circuits and Systems II, vol. 50, no. 10, pp. 728- 741, Oct. 2003.

[21] J. Roderick, H. Krishnaswamy, K. Newton and H. Hashemi, "Silicon-Based Ultra-Wideband Beam-Forming," IEEE J. SolidState Circuits, vol. 41, no. 8, pp. 1726- 1739, Aug. 2006.

[22] H. Veenstra, M. Notten, D. Zhao and J. R. Long, "A 3-channel true-time delay transmitter for $60 \mathrm{GHz}$ radar-beamforming applications," ESSCIRC Proc., 2011, pp. 143-146.

[23] T. Chu and H. Hashemi, "A true time-delay-based bandpass multi-beam array at mm-waves supporting instantaneously wide bandwidths," IEEE ISSCC Dig. Tech. Papers, 2010, pp. 38-39.

[24] A. C. Ulusoy, B. Schleicher and H. Schumacher, "A Tunable Differential All-Pass Filter for UWB True Time Delay and Phase Shift Applications," IEEE Microwave and Wireless Components Letters, pp. 462-464, 2011, vol. 21, no. 9. 
Chapter 6. Compact Cascadable gm-C All-Pass True Time Delay Cell with Reduced Delay Variation over Frequency

[25] E. Adabi and A. M. Niknejad, "Broadband variable passive delay elements based on an inductance multiplication technique," IEEE RFIC Symposium, 2008, pp. 445- 448.

[26] Q. Ma, R. Mahmoudi and D. M. W. Leenaerts, "A 12ps truetime-delay phase shifter with $6.6 \%$ delay variation at 20 40GHz," IEEE RFIC Symposium, 2013, pp. 61-64. 


\section{Chapter 7 \\ Summary and Conclusion}

\subsection{The work and the results}

The subject of this thesis is the design of a wideband phased array antenna system via time delay blocks in CMOS technology. Linear phased array antenna systems have been analyzed and these are basic building blocks for 2D and 3D phased array systems. The operational mechanism and the functionality of their basic sub-blocks, like time delay blocks and summation/splitting units were explained in addition to a qualitative description of the array factor and the beam steering.

The mathematical analysis of phased array antenna systems provides a quantitative estimation of the array factor as a function of $\mathrm{N}$ (the number of the elements), $\mathrm{d}$ (the inter-element distances of the antennas), $\tau$ (the values of the time delays) and $f$ (the operating frequency of the system). The resulting expression is used to analyze/synthesize the array factor. As an example the simulated effects of main parameters on the array factor of a linear 4 antenna element array were shown.

The structure and operational mechanism of the hierarchical beamforming systems were explained, which provide plenty degrees of freedom in the design of a phased array both in structures and subblocks. The phenomenon of grating lobe suppression of the second layer via nulls of the first layer is explained. This is important in designing array factors with low side-lobe levels.

Different existing methods for on-chip delay implementations were covered, for example transmission lines, LC delay lines, $\mathrm{g}_{\mathrm{m}}-(\mathrm{R}) \mathrm{C}$ filters and digital delay cells. Choosing each delay cell entails going 
through the trade-offs between maximum possible delay, minimum delay steps, frequency, noise, power consumption, cost and size. For low $\mathrm{GHz}$ operation (under $3 \mathrm{GHz}$ ) no on-chip phased array antenna system operating via time delay cells has been reported to the knowledge of the author.

Phase shifters have been used as alternative blocks to approximate time delays in limited bandwidths. It was mentioned that using mixers is the most common method for the physical implementation of phase shifters. The implementation of phased array antenna systems via phase shifters instead of time delays causes the unwanted phenomenon of beam squinting. It causes the beam direction to be dependent on the operating frequency. Beam squinting causes bandwidth limitations in the phased antenna systems and vulnerability to spatial interferences. Besides, beam squinting in hierarchical beamforming systems results in a dramatic rise of the side-lobe levels which again causes vulnerability to the spatial interferences.

In addition, the advantages and disadvantages of CMOS technology for the implementation of the sub-blocks of the phased array antenna were discussed. CMOS transistors are high speed, small size and low cost. The excellent switching property of CMOS transistors (which are mainly designed for digital circuits) make them quite suitable for implementation of switching mixers and therefore high performance phase shifters. Hence, the CMOS circuit designers are encouraged to implement phased array antenna systems via phase shifters. This consequently results in the beam squinting phenomenon and therefore narrow bandwidth of the phased arrays. This challenge guided this work toward the design of time delay blocks in CMOS technology. But implementation of time delay blocks in CMOS technology for low $\mathrm{GHz}$ applications requires different implementation methods than the use of bulky and expensive transmission lines and LC delay lines. It appears that $\mathrm{g}_{\mathrm{m}}-(\mathrm{R}) \mathrm{C}$ filters are promising solutions. These filters can approximate the ideal time delay cells in wide frequency ranges. By cascading the filters various amounts of delay are achievable. Also the delay tuning in these cells is feasible by tuning the values of the resistors and capacitors. But still the frequency dependency of delay in these filters can cause beam squinting in phased array antenna systems. 
To quantify the consistency of the produced delay with respect to frequency, the criterion $f_{\varphi=0}$ was introduced. $f_{\varphi=0}$ for each operating point is the crossing point of the frequency axis and the tangent line to the phase transfer function of the delay block. An expression was shown that estimates the delay vs. frequency variations of any delay cell, as a function of the bandwidth, operating frequency and $f_{\varphi=0}$ of the cell. $f_{\varphi=0}$ has a unique property: in a delay line of identical cascaded delay cells, $f_{\varphi=0}$ remains the same, independent of the number of cascaded cells. Therefore the delay vs. frequency variations of the total delay line, with any length, is easily calculable simply via $f_{\varphi=0}$ of its constituent small delay cells. This property is useful in phased array antenna systems to characterize the delay versus frequency variations of the variable delays of the antenna channels. It has led to the extraction of a mathematical expression for quantifying the beam squinting of a phased array antenna system as a function of the $f_{\varphi=0}$ of the individual delay cells. In synthesis, the expression provides an estimation of the $f_{\varphi=0}$ of the individual delay cells using information about the allowed amount of beam squinting.

For two reported $\mathrm{g}_{\mathrm{m}}-(\mathrm{R}) \mathrm{C}$ filters (delay cells) in literature, suitable for low supply voltage $(1.8 \mathrm{~V})$, the implementation has been analyzed. Both filters are implementations of $1^{\text {st }}$ order all-pass filters to approximate time delays up to a wide frequency range. They are named here "classical" and "Buckwalter" delay cells. The differences between their topologies result in different location of parasitic capacitances in them and practically higher order poles that cause gain roll-off and extra delay. Both filter topologies have floating capacitors and their plate-to-substrate parasitic capacitors are the main cause of extra unwanted poles. Also, they are not directly cascade-able to make delay lines because their input/output dc levels are different and they have an AC loading effect on each other. Therefore, dc blocking capacitors and buffers are needed to isolate them from each other, which results in more parasitic capacitors.

A method has been introduced to compare the useful frequency range of different $1^{\text {st }}$ order $\mathrm{g}_{\mathrm{m}}$-RC delay cell topologies. In this method the cells are designed to provide their maximum possible pole frequency $\left(\mathrm{f}_{\mathrm{p}}\right)$ with the condition that the gain roll-off $\left(\Delta \mathrm{H}_{\mathrm{p}}\right)$ of the cell at its pole frequency remains below a certain fixed value (for example 
$1 \mathrm{~dB})$. Because the parasitic capacitors depend on the implemented topology, the maximum possible $\mathrm{f}_{\mathrm{p}}$ depends on the topology. Simulation results on both "classical" and "Buckwalter" delay cells implemented in $160 \mathrm{~nm}$ and $180 \mathrm{~nm}$ CMOS technologies showed that with the condition of $\Delta \mathrm{H}_{\mathrm{p}}<1 \mathrm{~dB}$, the useful possible frequency range (the frequency range that the gain roll-off is less than $1 \mathrm{~dB}$ ) is less than $1 \mathrm{GHz}$.

To have delay cells suitable for high frequency ranges up to a few $\mathrm{GHz}$, a new topology for a $1^{\text {st }}$ order all-pass filter was developed. The topology was designed with non-floating capacitors and less parasitic capacitor effects. The dc level of the input and output are the same and the dc input impedance of the filter is infinite, therefore the filter is directly cascade-able in a delay line without the need for DC blocking capacitors and inter-stage buffers. This has resulted in less parasitic capacitors. The current reuse in the filter results in low power consumption. The characteristics of the filter with respect to noise, nonlinearity and PVT sensitivity were analyzed. Several techniques were performed on the filter to improve its characteristics: A) Its bandwidth has been extended via active inductive peaking at the output of the filter, B) Its phase vs. frequency was linearized to make the delay consistent over wide frequency bands. $\mathrm{f}_{\varphi=0}$ was used to quantify the phase linearization. C) The delay tuning structure was added to the filter, consisting of a set of switched capacitors. It provides the possibility of small delay steps in the filter. D) A gain tuning structure was added to the filter to calibrate its DC gain to unity without changing the DC operating points of the filter. The result is a cascade-able compact $\mathrm{g}_{\mathrm{m}}-\mathrm{C}$ all-pass filter cell for 1-2.5 GHz. Compared to previous $\mathrm{g}_{\mathrm{m}}-\mathrm{RC}$ and $\mathrm{g}_{\mathrm{m}}-\mathrm{C}$ filter cells, it achieves at least a $5 \mathrm{x}$ larger frequency range for the same amount of delay, while keeping gain variations within $1 \mathrm{~dB}$.

Finally, a $160 \mathrm{~nm}$ CMOS chip with a maximum delay of $550 \mathrm{psec}$ is demonstrated with monotonous delay steps of 13 psec ( 41 steps) and an RMS delay variation error of less than 10psec (only 1.8\% of the realized delay) over more than an octave in frequency $(1-2.5 \mathrm{GHz})$. The delay per area is at least 50x more than found in literature (refer to table 6.3). The all-pass cells were used to realize a four-element phased array receiver IC. Measurement results of the beam pattern 
demonstrate the wideband operation capability of the $\mathrm{g}_{\mathrm{m}}-\mathrm{RC}$ time delay cell and the phased array IC architecture.

\subsection{Original contribution}

1. A new criterion $\left(\mathrm{f}_{\varphi=0}\right)$ has been introduced to quantify delay versus frequency variations in time delay blocks.

2. A general expression to quantify beam squinting in phased array antenna systems as a function of $\mathrm{f}_{\varphi=0}$ of its constituent delay cells has been derived.

3. A method to benchmark and compare the existing $1^{\text {st }}$ order $\mathrm{g}_{\mathrm{m}}-(\mathrm{R}) \mathrm{C}$ delay cells has been introduced.

4. A new circuit topology for a $1^{\text {st }}$ order all-pass $g_{m}-(R) C$ filter has been developed which behaves as a delay cell with $5 \mathrm{X}$ improvement of the useful frequency range compared to existing $1^{\text {st }}$ order $g_{m}-(R) C$ filters.

5. A phase linearization technique to reduce delay vs. frequency variations in the proposed delay cell has been implemented

6. A chip architecture for wideband phased array antenna systems based on the $\mathrm{g}_{\mathrm{m}}-(\mathrm{R}) \mathrm{C}$ filters (proposed delay cells) has been designed and implemented in $160 \mathrm{~nm}$ CMOS technology.

\subsection{Recommendations for future work}

1. The proposed $1^{\text {st }}$ order $\mathrm{g}_{\mathrm{m}}-\mathrm{C}$ circuit topology can be implemented in other technologies, for example in technologies with transistors having higher intrinsic gains $\left(g_{m} r_{0}\right)$ than CMOS technologies or even higher unity current gains $\left(f_{T}\right)$. In such technologies the effect of finite output impedances of transistors or the effect of parasitic capacitors on the transfer function of the filter is lower (compared to CMOS) and the filters can operate at higher frequencies. However supply voltage range requirements, linearity and 
noise and power tradeoff of the filter implemented in the new technology have to be compared with the filter implemented in CMOS technology.

2. Using cascoded transistors in the structure of the filter reduces the effect of finite output impedance of the transistors on the transfer function of the filter. However it can limit the voltage head room at the output of the filter and also results in some extra poles or maybe affects the linearity of the filter.

3. The non-linearity of the proposed $1^{\text {st }}$ order $g_{m}-C$ filter mainly originates from the reactive harmonic distortion of the current mirror. By using source degeneration resistors in the PMOS current mirrors, the linearity of the current mirrors improves at the expense of less voltage headroom. Therefore a trade-off appears between linearity and bandwidth of the filter. Finding an optimum value of the source degeneration resistors results in the best linearity while keeping the bandwidth still high.

4. The bandwidth of the chip is also limited by the parasitic capacitors (to the substrate) of the dc blocking capacitors of the LNA/BALUN block. An improved topology of LNA/BALUN which omits the dc-blocking capacitors results in more bandwidth for the chip. 


\section{List of Publications}

1. S. K. Garakoui, E. A. M. Klumperink, B. Nauta and F. E. van Vliet, "Time delay circuits: A quality criterion for delay variations versus frequency," IEEE ISCAS Proc., 2010, pp. 4281-4284.

2. S. K. Garakoui, E. A. M. Klumperink, B. Nauta and F. E. van Vliet, "Phased-array antenna beam squinting related to frequency dependency of delay circuits," EuRad Conf., 2011, pp. 416-419.

3. S. K. Garakoui, E. A. M. Klumperink, B. Nauta and F. E. van Vliet, "A 1-to-2.5GHz phased-array IC based on gm-RC all-pass time-delay cells," IEEE ISSCC Dig. Tech. Papers, 2012, pp. 8082.

4. F.E. van Vliet, E.A.M. Klumperink, M. Soer, S.K. Garakoui, A. de Boer, A.P. de Hek, W.de Heij, B Nauta, "Advance in silicon phased-array receiver IC's”, IEEE MTT-S , Microwave Symposium Dig. (MTT), 2012, pp.1-3.

5. S. K. Garakoui, E. A. M. Klumperink, B. Nauta and F. E. van Vliet, "Frequency Limitations of First-Order gm - RC All-Pass Delay Circuits," IEEE T. on Circuits and Systems II, vol. 60, no. 9, pp. 572-576, Aug. 2013.

6. S. K. Garakoui, E. A. M. Klumperink, B. Nauta and F. E. van Vliet, " Compact Cascadable gm-C All-Pass True Time Delay Cell With Reduced Delay Variation Over Frequency," IEEE Journal of Solid-State Circuits, vol. 50, no. 3, pp. 693-703, March. 2015. 